\title{
Clustering and the Three-Point Function
}

\author{
Yunfeng Jiang ${ }^{a}$, Shota Komatsu ${ }^{b}$, Ivan Kostov $^{c 1}$, Didina Serban $^{c}$ \\ a Institut für Theoretische Physik, ETH Zürich, \\ Wolfgang Pauli Strasse 27, CH-8093 Zürich, Switzerland \\ ${ }^{b}$ Perimeter Institute for Theoretical Physics, \\ Waterloo, Ontario, Canada \\ ${ }^{c}$ Institut de Physique Théorique, DSM, CEA, URA2306 CNRS \\ Saclay, F-91191 Gif-sur-Yvette, France \\ jiangyf2008@gmail.com, skomatsu@perimeterinstitute.ca, \\ ivan.kostov \& didina.serban@cea.fr
}

\begin{abstract}
We develop analytical methods for computing the structure constant for three heavy operators, starting from the recently proposed hexagon approach. Such a structure constant is a semiclassical object, with the scale set by the inverse length of the operators playing the role of the Planck constant. We reformulate the hexagon expansion in terms of multiple contour integrals and recast it as a sum over clusters generated by the residues of the measure of integration. We test the method on two examples. First, we compute the asymptotic three-point function of heavy fields at any coupling and show the result in the semiclassical limit matches both the string theory computation at strong coupling and the tree-level results obtained before. Second, in the case of one non-BPS and two BPS operators at strong coupling we sum up all wrapping corrections associated with the opposite bridge to the non-trivial operator, or the "bottom" mirror channel. We also give an alternative interpretation of the results in terms of a gas of fermions and show that they can be expressed compactly as an operatorvalued super-determinant.
\end{abstract}

\footnotetext{
${ }^{1}$ Associate member of the Institute for Nuclear Research and Nuclear Energy, Bulgarian Academy of Sciences, 72 Tsarigradsko Chaussée, 1784 Sofia, Bulgaria
} 


\section{Contents}

1 Introduction 2

2 The three-point function and the hexagon proposal 4

2.1 Results and comparison with strong coupling . . . . . . . . . . . . . 7

3 Asymptotic structure constant for two BPS and one non-BPS operator 10

3.1 From sum-over-partition to multiple contour integral . . . . . . . . . . . 11

3.2 Tree-level revisited . . . . . . . . . . . . . . . . . . . 13

3.3 Deformation of contours and clustering . . . . . . . . . . . . . . 15

3.4 The exact result and semiclassical limit . . . . . . . . . . . . . . . . . 18

3.5 The semiclassical limit for the $\mathfrak{s u}(2)$ sector . . . . . . . . . . . . . . . . 20

3.6 The semiclassical limit for the $\mathfrak{s l}(2)$ sector . . . . . . . . . . . . . . . . . 21

4 Asymptotic structure constant for three non-BPS fields 22

4.1 Formulation in terms of multiple contour integrals . . . . . . . . . . . . . 23

4.2 Taking the semiclassical limit, $\mathfrak{s u}(2) \ldots \ldots \ldots$

4.3 Taking the semiclassical limit, $\mathfrak{s l}(2) \ldots \ldots \ldots$. . . . . . . . . . . 28

5 Bottom mirror excitations $r$

5.1 Quantities for bound states at strong coupling . . . . . . . . . . . . 30

5.2 Clustering the mirror particles . . . . . . . . . . . . . . 36

5.3 The $\mathfrak{s u}(2)$ bottom mirror contribution . . . . . . . . . . . . . . . . . . 39

5.4 The $\mathfrak{s l}(2)$ bottom mirror contribution . . . . . . . . . . . . . . . . 40

6 Fredholm determinants and free fermions 41

6.1 Multiple contour integrals as a Fredholm determinant . . . . . . . . . . . . 43

6.2 The leading term by Fredholm determinant . . . . . . . . . . . . . . . . . 44

6.3 The leading term as the free energy of a Fermi gas . . . . . . . . . . . . 44

6.4 Semiclassical expansion by nested Fredholm determinant . . . . . . . . . . 45

6.5 CFT representation . . . . . . . . . . . . . . . . . . 47

6.6 Wrapping corrections by Fredholm determinant . . . . . . . . . . . . 47 
A An example for clustering $\quad 54$

B Scaling limit of the $\mathfrak{s l}(2)$ and $\mathfrak{s u}(2)$ scalar factors $\quad 54$

C Computing the phase factor for the asymptotic $C_{123}^{\circ \cdots \bullet} \quad 55$

D The analytic structure of the transfer matrices in mirror kinematics $\quad 58$

$\begin{array}{lll}\mathrm{E} & \text { The term } \mathrm{B}_{3} \text { at strong coupling } & 61\end{array}$

F $\quad$ The ABJM matrix model and clustering $\quad 62$

$\begin{array}{ll}\text { G Separation of variables from clustering } & 64\end{array}$

\section{Introduction}

In the strongly-interacting system with a large number of degrees of freedom, it is often the case that the system exhibits emergent collective behaviour, which is entirely different from that of its constituents and provides us with a novel physical picture. The examples of such range from various condensed-matter systems realised in the laboratory, to the AdS/CFT correspondence, which claims that the strongly-coupled CFTs satisfying certain conditions can be described by the gravitational theory in the AdS spacetime.

In this paper, we address one simple but intriguing example of such phenomena in the context of the AdS/CFT correspondence; namely the emergence of the classical string worldsheet from the three-point functions in the planar $\mathcal{N}=4$ super Yang-Mills theory (SYM). On the one hand, a non-perturbative framework to compute the three-point functions of $\mathcal{N}=4 \mathrm{SYM}$, called the hexagon vertex, was put forward recently in [1]. It describes the three-point functions in terms of the dynamics of "magnons", which are the elementary fields constituting the gauge-invariant operator. On the other hand, the AdS/CFT implies that the very same object in the strong coupling limit admits a totally different description in terms of the classical string worldsheet and that the three-point function is given by its area [2-5]. However, apart from some partial results given in [1], it is still not clear whether and how these two descriptions are consistent with each other.

The main purpose of this paper is to explore the general mechanism which connects 
these two results. We claim that the semiclassical regime is achieved through a mechanism which we call clustering. When a large number of magnons are put together in the hexagon vertex, they form a sort of bound states, which we call clusters. As we demonstrate in several examples, this clustering phenomenon is essential in order to reproduce the stringtheory results from the hexagon vertex. It is worth noting that these clusters bear some resemblance with the bound states in the context of the Thermodynamic Bethe Ansatz.

In order to explain more in detail what we computed with this method, let us briefly recall the structure of the hexagon vertex and the result from the classical string. The hexagon vertex consists of two parts: The one is the asymptotic structure constant which is given by a sum over partitions of the magnons and describes the three-point functions of long operators. The other is the wrapping corrections, which is given by the sums and the integrals of the mirror particles and accounts for the finite size effects. On the other hand, the result from the string theory is given in terms of integrals on the spectral curve, where the integration contours are either around the branch cuts or around the unit circle.

Let us now describe what we achieved in this paper. First we study the asymptotic three-point function of long non-BPS operators in the rank one sectors and show that the result after clustering reproduces the integrals around the branch cuts in the string-theory prediction. Second, in the case of the one non-BPS and two BPS correlators, we sum up the wrapping corrections associated with the edge opposing to the non-BPS operator, taking into account the clustering effect. The result matches nicely with one of the integrals around the unit circle in the string-theory computation.

Our analysis is based upon yet another important observation that, in the regimes of our interest, the expression coming from the hexagon vertex takes the form of the grandcanonical partition function of free fermions. This allows to apply the methods developed in [6] and in [7-9] for the tree-level correlators. When the number of magnons is infinite, these fermions become classical and the result is given by the phase-space integral of this fermion system, which matches the string-theory prediction. This Fermi gas description allows to reproduce the results obtained by clustering in an elegant way, shortcutting the tedious combinatorics. Furthermore, it reveals that the sum over mirror particles on the bottom edge can be nicely re-expressed as the operatorial superdeterminant. However the derivation based on the Fermi gas is not, at the present stage, sufficiently rigorous. Therefore, for the most parts of the paper, we stay on the safe ground of the clustering method and only briefly sketch the Fermi gas approach.

The applicability of these two approaches is not limited to the three-point functions. For instance, the clustering method has proven to be useful for various other problems such as the strong coupling limit of the scattering amplitudes in $\mathcal{N}=4 \mathrm{SYM}$ [10], which 
was otherwise obtained by different methods [11, 12]. Clustering-like methods were used to compute the partition functions in $\mathcal{N}=2$ gauge theories in the Nekrasov-Shatashvilli limit [13-15], and the integrable models describing non-equilibrium processes [16-18]. On the other hand, the Fermi gas approach is used extensively to study the M-theoretic large $N$ limit of the ABJM and related theories as well as the super-conformal index in four dimensions $[19,20]$. Our analysis indicates that these approaches are deeply connected.

The rest of the paper is structured as follows. In section 2 we review the computation of the three-point function and the hexagon vertex and summarise our results, as well as the string-theory prediction at strong coupling [21]. Then in section 3 we study the asymptotic structure constant for the three-point function of one non-BPS and two BPS operators. For this purpose, we first re-express the sum-over-partitions formula in the hexagon proposal as a multiple contour integral. We then explain the basic idea of the clustering using the tree-level example and show that the method can be applied at finite coupling. Next, in section 4 we generalise it to the case of three non-BPS fields and reproduce the string-theory prediction. In section 5, we turn to the wrapping corrections and summarise expressions for the basic quantities at strong coupling. Using such expressions, we analyse the clustering of the mirror particles and obtain the expression consistent with the string theory. Lastly in section 6 , we show that these results can be computed alternatively using the Fermi gas approach and the Fredholm determinant. We in particular show that the summation over the mirror particles can be expressed as the generalised Fredholm determinant ${ }^{2}$, which can be further converted into an operator-valued superdeterminant. We conclude in section 7. Several appendices are provided in order to explain technical details and elucidate the relation between the clustering and other methods: In Appendix F, we study the ABJM matrix model using the clustering method, and in Appendix G, we relate the hexagon vertex and the separation of variables at tree-level using the clustering.

\section{The three-point function and the hexagon proposal}

The three-point function of operators in the $\mathcal{N}=4$ planar SYM theory is fixed up to a constant by the conformal invariance,

$$
\left\langle\mathcal{O}_{1}\left(x_{1}\right) \mathcal{O}_{2}\left(x_{2}\right) \mathcal{O}_{3}\left(x_{3}\right)\right\rangle=\frac{C_{123}(g)}{\left|x_{12}\right|^{\Delta_{12}}\left|x_{13}\right|^{\Delta_{13}}\left|x_{23}\right|^{\Delta_{23}}},
$$

with $x_{i}$ vectors in the $3+1$ dimensional Minkowski space, $\Delta_{i}$ the conformal dimension of the operator $\mathcal{O}_{i}$ and $\Delta_{i j}=\Delta_{i}+\Delta_{j}-\Delta_{k}$. The constant $C_{123}$ is given in terms of the initial

\footnotetext{
${ }^{2}$ The generalised Fredholm determinant is introduced originally in the context of topological strings [22].
} 
data of the three operators, namely the charges of the global symmetry group $P S U(2,2 \mid 4)$ and the charges of the infinite symmetry group associated to integrability. The latter ones, dependent on the coupling constant $g$, can be encapsulated, at least in the regime of in the small $g$, by three collections of rapidities $\mathbf{u}_{1}, \mathbf{u}_{2}, \mathbf{u}_{3}$, each associated to one of the operators $\mathcal{O}_{1}\left(x_{1}\right), \mathcal{O}_{2}\left(x_{2}\right), \mathcal{O}_{3}\left(x_{3}\right)$. At $g=0$ the three sets of rapidities are determined by Bethe ansatz equations for three $P S U(2,2 \mid 4)$ spin chains with lengths $L_{1}, L_{2}$ and $L_{3}$. At non-zero values of the coupling constant $g$, the spin chains acquire long-range interaction and the so-called asymptotic Bethe ansatz is not exact anymore. The long-range corrections can be interpreted as coming from virtual particles circulating in the so-called mirror channel, where time and space are interchanged. These virtual particles are called mirror particle. Their contribution to the spectrum of conformal dimensions $\Delta(g)$ can be exactly determined via a set of functional equations known under the name of Quantum Spectral Curve, equivalent to a system of Thermodynamic Bethe Ansatz equations. In the large volume limit the contribution of the virtual particles is exponentially small.

Through the AdS/CFT correspondence [23], the three-point function is dual to a threestring interaction connecting three strings with energies $\Delta_{1}, \Delta_{2}, \Delta_{3}$. The rapidities can be then associated to the momenta of excitation modes, or magnons, propagating on the 1+1 dimensional worldsheet. For a particular subset of the operators, the BPS operators, the conformal dimensions do not depend on the coupling constant $g$ and the associated rapidities are trivial (i.e. infinite). We are going to use a bullet to symbolise a non-BPS operator and an empty circle to denote the BPS one with the same global charges. To remove some trivial combinatorial factors we are dividing the three-point function by the three-point function of the corresponding BPS operators, e.g.

$$
\mathcal{C}_{123}^{\bullet \bullet \circ} \equiv \frac{C_{123}^{\bullet \bullet \circ}}{C_{123}^{\circ \circ \circ}} \sqrt{\mathcal{N}_{1} \mathcal{N}_{2}}
$$

denotes the three-point function of two non-BPS and one BPS operator. In the above formula, $\sqrt{\mathcal{N}_{i}}$ are the normalisation of the three incoming states, which can be expressed in terms of the Gaudin determinants. In this work we are not considering the explicit expression of the norms, and prefer considering the unnormalised structure constants $\mathcal{C}_{123}$ defined in (2.2) instead of the normalised structure constants $C_{123}$. The semiclassical limit of the norms in the absence of mirror correction was taken in $[7,24]$.

An all-loop prescription to compute the three-point function was given in [1]. The guiding principle of the proposal is to split the worldsheet of the three interacting strings into two overlapping hexagons, and then sum over all possible ways of distributing the magnon excitations between the two hexagons, $\mathbf{u}_{1}=\alpha_{1} \cup \bar{\alpha}_{1}, \mathbf{u}_{2}=\alpha_{2} \cup \bar{\alpha}_{2}, \mathbf{u}_{3}=\alpha_{3} \cup \bar{\alpha}_{3}$ as illustrated in figure 2.1. In the absence of the mirror corrections (asymptotic limit) the 
answer is
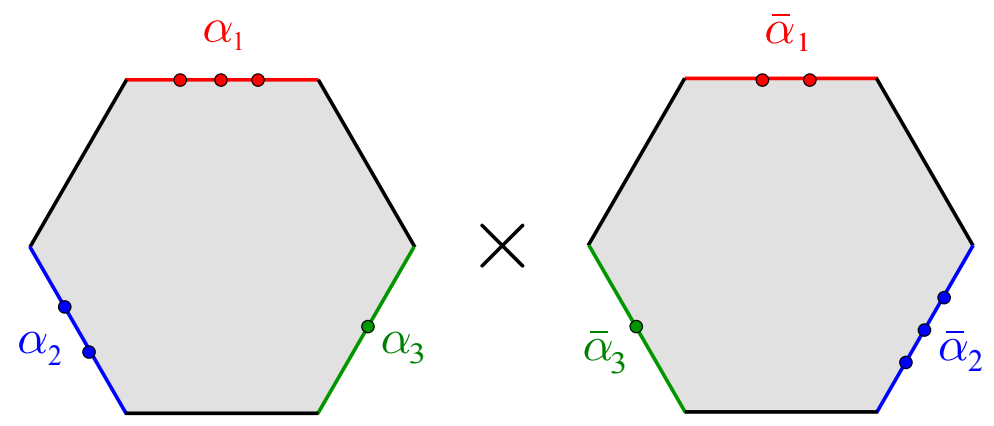

Figure 2.1: A possible arrangement of excitations for the hexagon form factors.

$$
\begin{aligned}
{\left[\mathcal{C}_{123}^{\bullet \bullet \bullet}\right]^{\text {asympt }} } & =\sum_{\alpha_{i} \cup \overline{\alpha_{i}}=\mathbf{u}_{i}} \prod_{i=1}^{3}(-1)^{\left|\alpha_{1}\right|+\left|\alpha_{2}\right|+\left|\alpha_{3}\right|} w_{\ell_{31}}\left(\alpha_{1}, \bar{\alpha}_{1}\right) w_{\ell_{12}}\left(\alpha_{2}, \bar{\alpha}_{2}\right) w_{\ell_{23}}\left(\alpha_{3}, \bar{\alpha}_{3}\right) \\
& \times \quad \mathrm{H}\left(\alpha_{1}\left|\alpha_{3}\right| \alpha_{2}\right) \mathrm{H}\left(\bar{\alpha}_{2}\left|\bar{\alpha}_{3}\right| \bar{\alpha}_{1}\right) .
\end{aligned}
$$

Explicit expressions for transition factors $w_{\ell_{i-1, i}}\left(\alpha_{i}, \bar{\alpha}_{i}\right)$ and hexagon form factors $\mathrm{H}\left(\alpha_{1}\left|\alpha_{3}\right| \alpha_{2}\right)$ were proposed in [1] and will be given later. The building blocks of the hexagon form factors are the bi-local hexagon amplitudes $h(u, v)$ proposed in [25] and the elements of the Beisert's scattering matrix [26]. Here we are going to consider only structure constants of operators from the rank-one sectors $\mathfrak{s u}(2)$ and $\mathfrak{s l}(2)$ and we are therefore not going to use the matrix structure of the hexagon form factors.

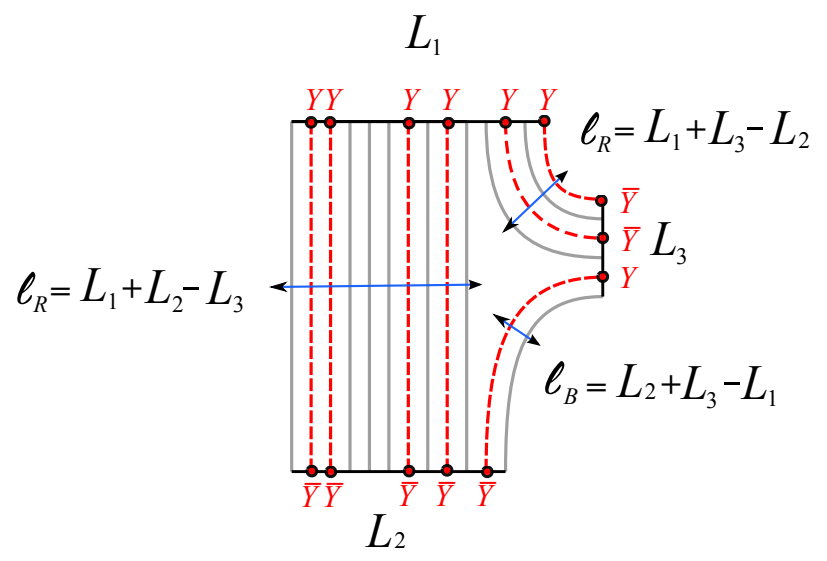

Figure 2.2: Vacua and $\mathfrak{s u}(2)$ excitations in the reservoir picture of BKV [1].

To connect with the weak-coupling picture and the corresponding notations, it is useful to represent the three-point function we consider in the reservoir picture of [1] represented 
in 2.2. In this picture, the first operator $\mathcal{O}_{1}$ is of the form $\operatorname{Tr}\left(Z^{L_{1}-M_{1}} Y^{M_{1}}\right)+\ldots$, the second operator $\mathcal{O}_{2}$ is of the form $\operatorname{Tr}\left(\bar{Z}^{L_{2}-M_{2}} \bar{Y}^{M_{2}}\right)+\ldots$, and the third operator $\mathcal{O}_{3}$, the reservoir, is built as $\operatorname{Tr}(Z+\bar{Z}+Y-\bar{Y})^{L_{3}-M_{3}}(\bar{Y}-\bar{Z})^{M_{3}}+\ldots$. This type of structure constant is called type I-I-II in [27], since two operators belong to the "left" $\mathfrak{s u}(2)$ sector and one belongs to the "right" $\mathfrak{s u}(2)$ sector in the sense that the operator $\mathcal{O}_{2}$ can be obtained from $\operatorname{Tr}\left(Z^{L_{2}} Y^{M_{2}}\right)+\ldots$ and $\mathcal{O}_{3}$ from $\operatorname{Tr}\left(Z^{L_{3}-M_{3}} \bar{Y}^{M_{3}}\right)+\ldots$ by one of the twisted translation defined in [28] and used in [1]. A similar definition works in the $\mathfrak{s l}(2)$ sector.

The inclusion of wrapping corrections to equation (2.3) is done by including an infinite tower of excitations, as well as their bound states, circulating in the three mirror channels denoted by black edges in figure 2.1. The summation is done over their rapidities and their polarisations. The general expression is too complicated to be reproduced here; instead, we can illustrate the type of contribution on the case of a single non-BPS operator. We consider only the mirror particles in the channel opposed to that operator, as showed in figure 2.3. Following [25] we call this channel the bottom channel. In this case, the asymptotic and mirror contributions conveniently factorise,

$$
\mathcal{C}^{\bullet \circ \circ}=\left[\mathcal{C}^{\bullet \circ \circ}\right]^{\text {bottom }}\left[\mathcal{C}^{\bullet \circ \circ}\right]^{\text {asympt }}
$$

Schematically, given in terms of only the fundamental excitations, the expression of the wrapping corrections is given by $[1,25]$

$$
\left[\mathcal{C}^{\bullet \circ \circ}\right]^{\text {bottom }}=\int_{-\infty}^{\infty} d \mathbf{w} \mu\left(\mathbf{w}^{\gamma}\right) e^{i p\left(\mathbf{w}^{\gamma}\right) \ell_{B}} T\left(\mathbf{w}^{\gamma}\right) h^{\neq}\left(\mathbf{w}^{\gamma}, \mathbf{w}^{\gamma}\right) h\left(\mathbf{u}, \mathbf{w}^{-3 \gamma}\right)
$$

with $\ell_{B}=\frac{1}{2}\left(L_{2}+L_{3}-L_{1}\right)$ the length of the bottom bridge of the correlator, opposed to the operator $\mathcal{O}_{1}$ and $T(w)$ the $\mathfrak{s u}(2 \mid 2)$ spin chain transfer matrix [26]. The full result takes into account all the bound states and will be given in the corresponding section. Here and below the index $\gamma$ stands for the mirror transformation and we use the shorthand notations

$$
h(\mathbf{u}, \mathbf{v}) \equiv \prod_{i, j} h\left(u_{i}, v_{j}\right), \quad h^{\neq}(\mathbf{u}, \mathbf{u})=\prod_{i \neq j} h\left(u_{i}, u_{j}\right)
$$

\subsection{Results and comparison with strong coupling}

In the case when the incoming operators correspond to semiclassical strings, the lengths $L_{1}, L_{2}, L_{3}$ of the three chains and the numbers of the magnon excitations $M_{1}, M_{2}, M_{3}$ are large. The semiclassical limit is controlled by a small parameter $\epsilon$ such that $\epsilon L_{i}$ and $\epsilon M_{i}$ remain finite when $\epsilon \rightarrow 0$. This limit exists for any value of the 't Hooft coupling $g$. In addition to the semiclassical limit, one can take the strong coupling limit where the effective 


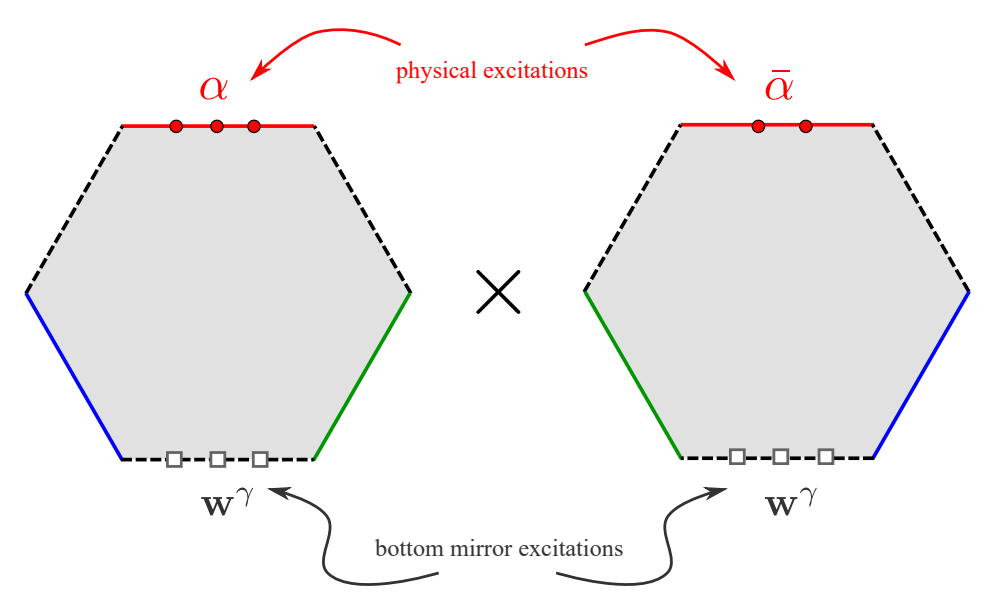

Figure 2.3: The physical and bottom mirror excitations.

coupling $g^{\prime}=\epsilon g$ remains finite when $\epsilon \rightarrow 0$. Based on the experience with the spectrum [29], we may expect that, for $\mathfrak{s l}(2)$, the results for the semiclassical strings can be applied safely to small values of $\epsilon L_{i}$.

The summation over the different ways of partitioning the rapidities in equation (2.3), as well as the summation over the mirror particles remains an open problem in general. Here we report some modest progress in taking the sum and the semiclassical limit in three particular cases when the operators belong to the rank-one sectors $\mathfrak{s u}(2)$ and $\mathfrak{s l}(2)$ :

- the expression of the asymptotic part of the structure constant for one non-BPS and two BPS operators, $\left[\mathcal{C}^{\bullet \circ}\right]^{\text {asympt }}$ for any value of the coupling constant,

- the expression of the asymptotic part of the I-I-II structure constant ${ }^{3}$ for three nonBPS operators belonging to two different $\mathfrak{s u}(2)$ or $\mathfrak{s l}(2)$ sectors, $\left[\mathcal{C}^{\bullet \bullet \bullet}\right]^{\text {asympt }}$, for any value of the coupling constant,

- the expression of the bottom mirror contribution for one non-BPS and two BPS operators, $\left[\mathcal{C}^{\bullet \circ}\right]^{\text {bottom }}$ in the strong coupling limit.

The first case is a relatively simple generalisation of the result obtained by $[6,7,9]$ at treelevel. Here we use a slightly different method of taking the semiclassical limit, based on an integral representation of the sums in (2.3) which has already appeared in [9]. This method is alternative to the Fredholm determinant method used there and it is easily adaptable to situation when the structure constant cannot be written exactly as a determinant. Finally, the structure of the integrals in the third case ressemble strongly that from the first two

\footnotetext{
3 The I-I-I type structure constant remains out of reach of our method for the moment.
} 
cases, and we are able to take the sum over bound states exactly in the strong coupling limit.

The answer for the semiclassical structure constants is given in terms of quasi-momenta associated to the three operators, which encode the corresponding rapidities. For operators duals to semiclassical strings, the rapidities are distributed on a set of cuts, which connect different sheets of the quasi-momenta. We are going do denote by $\tilde{p}^{(k)}$ the sphere part and by $\hat{p}^{(k)}$ the AdS part of the quasi-momentum associated to the operator $\mathcal{O}_{k}$. The definition of the quasi-momenta will be given in the main text. The results for the $\mathfrak{s u}(2)$ and $\mathfrak{s l}(2)$ sectors are

$$
\begin{aligned}
& \log \left[\mathcal{C}_{123}^{\bullet \bullet \bullet}\right]_{\mathfrak{s u}(2)}^{\text {asympt }}=-\frac{1}{\epsilon} \oint_{\mathcal{C}_{\mathbf{u}_{1} \cup \mathbf{u}_{2}}} \frac{d u}{2 \pi} \operatorname{Li}_{2}\left[e^{i \tilde{p}_{L}^{(1)}+i \tilde{p}_{L}^{(2)}-i \tilde{p}_{R}^{(3)}}\right]-\frac{1}{\epsilon} \oint_{\mathcal{C}_{\mathbf{u}_{3}}} \frac{d u}{2 \pi} \operatorname{Li}_{2}\left[e^{i \tilde{p}_{R}^{(3)}+i \tilde{p}_{L}^{(2)}-i \tilde{p}_{L}^{(1)}}\right], \\
& \log \left[\mathcal{C}_{123}^{\bullet \bullet \bullet \bullet}\right]_{\mathfrak{s}(2)}^{\text {asympt }}=\frac{1}{\epsilon} \oint_{\mathcal{C}_{\mathbf{u}_{1} \cup \mathbf{u}_{2}}} \frac{d u}{2 \pi} \operatorname{Li}_{2}\left[e^{i \hat{p}_{L}^{(1)}+i \hat{p}_{L}^{(2)}-i \hat{p}_{R}^{(3)}}\right]+\frac{1}{\epsilon} \oint_{\mathcal{C}_{\mathbf{u}_{3}}} \frac{d u}{2 \pi} \operatorname{Li}_{2}\left[e^{i \hat{p}_{R}^{(3)}+i \hat{p}_{L}^{(2)}-i \hat{p}_{L}^{(1)}}\right] .
\end{aligned}
$$

where $\mathcal{C}_{\mathbf{u}_{k}}$ is a contour encircling counterclockwise the support of the rapidities $\mathbf{u}_{k}$. The result for $\left[\mathcal{C}_{123}^{\bullet \circ}\right]^{\text {asympt }}$ is the particular case where $\mathbf{u}_{2}=\mathbf{u}_{3}=\emptyset$. We would like to emphasise that the expression above are valid when the length of the three operators $L_{1}, L_{2}$ and $L_{3}$ are large and the supports of $\mathbf{u}_{1}, \mathbf{u}_{2}$ and $\mathbf{u}_{3}$ are well separated. The so-called heavy-heavy-light diagonal limit, when the length of one of the operators, say $L_{3}$, is small and in addition $\mathbf{u}_{1}=\mathbf{u}_{2}$ was studied in $[30,31]$.

A surprisingly similar form is taken by the result of the resummation of the virtual particles. Here we succeeded to take the sum only of the mirror particles for the structure constant with one non-BPS operator in the channel opposed to the non-trivial operator,

$$
\begin{aligned}
\log \left[\mathcal{C}_{123}^{\bullet \circ 0}\right]_{\mathfrak{s u}(2)}^{\text {bottom }} & =\frac{1}{\epsilon} \oint_{U} \frac{d u}{2 \pi}\left(\operatorname{Li}_{2}\left[e^{i\left(\hat{p}^{(2)}+\hat{p}^{(3)}-\hat{p}^{(1)}\right)}\right]-\operatorname{Li}_{2}\left[e^{i\left(\tilde{p}^{(2)}+\tilde{p}^{(3)}-\tilde{p}^{(1)}(x)\right)}\right]\right) \\
\log \left[\mathcal{C}_{123}^{\bullet \circ o}\right]_{\mathfrak{s}(2)}^{\text {bottom }} & =-\frac{1}{\epsilon} \oint_{U} \frac{d u}{2 \pi}\left(\operatorname{Li}_{2}\left[e^{i\left(\hat{p}^{(2)}+\hat{p}^{(3)}-\hat{p}^{(1)}\right)}\right]-\operatorname{Li}_{2}\left[e^{i\left(\tilde{p}^{(2)}+\tilde{p}^{(3)}-\tilde{p}^{(1)}(x)\right)}\right]\right)
\end{aligned}
$$

with the contour of integration $U$ encircling now the Zhukovsky cut with $u$ between $-2 g \epsilon$ and $2 g \epsilon$.

The three-point functions at strong coupling admit a completely different description, namely in terms of the area of the classical string worldsheet. The computation from the string theory side was completed recently building on earlier works [21]. In both $\mathfrak{s u}(2)$ and $\mathfrak{s l}(2)$ sectors, the result is composed of three terms,

$$
\log C_{123}^{\bullet \bullet \bullet}=\log \left[\mathcal{C}_{123}^{\bullet \bullet \bullet}\right]^{\text {asympt }}+\log \left[\mathcal{C}_{123}^{\bullet \bullet \bullet}\right]^{\text {wrapping }}+\text { Norm }
$$

For the type I-I-II three-point functions in the $\mathfrak{s u}(2)$ sector, the asymptotic part and the 
wrapping part are given on the string theory side by ${ }^{4}$

$$
\begin{aligned}
& \log \left[\mathcal{C}_{123}^{\bullet \bullet \bullet}\right]_{\mathfrak{s u}(2)}^{\text {asympt }}=-\frac{1}{\epsilon} \oint_{\mathcal{C}_{\mathbf{u}_{1} \cup \mathbf{u}_{2}}} \frac{d u}{2 \pi} \operatorname{Li}_{2}\left[e^{i \tilde{p}_{L}^{(1)}+i \tilde{p}_{L}^{(2)}-i \tilde{p}_{R}^{(3)}}\right]-\frac{1}{\epsilon} \oint_{\mathcal{C}_{\mathbf{u}_{3}}} \frac{d u}{2 \pi} \operatorname{Li}_{2}\left[e^{i \tilde{p}_{R}^{(3)}+i \tilde{p}_{L}^{(2)}-i \tilde{p}_{L}^{(1)}}\right], \\
& \log \left[\mathcal{C}_{123}^{\bullet \bullet \bullet}\right]_{\mathfrak{s u}(2)}^{\text {wrapping }}=\frac{1}{\epsilon} \oint_{U} \frac{d u}{2 \pi}\left(\operatorname{Li}_{2}\left[e^{i\left(\hat{p}^{(1)}+\hat{p}^{(2)}-\hat{p}^{(3)}\right)}\right]-\operatorname{Li}_{2}\left[e^{i\left(\tilde{p}_{L}^{(1)}+\tilde{p}_{L}^{(2)}-\tilde{p}_{R}^{(3)}\right)}\right]\right) \\
& +\frac{1}{\epsilon} \oint_{U} \frac{d u}{2 \pi}\left(\operatorname{Li}_{2}\left[e^{i\left(\hat{p}^{(2)}+\hat{p}^{(3)}-\hat{p}^{(1)}\right)}\right]-\operatorname{Li}_{2}\left[e^{i\left(\tilde{p}_{L}^{(2)}+\tilde{p}_{R}^{(3)}-\tilde{p}_{L}^{(1)}\right)}\right]\right) \\
& +\frac{1}{\epsilon} \oint_{U} \frac{d u}{2 \pi}\left(\operatorname{Li}_{2}\left[e^{i\left(\hat{p}^{(3)}+\hat{p}^{(1)}-\hat{p}^{(2)}\right)}\right]-\operatorname{Li}_{2}\left[e^{i\left(\tilde{p}_{R}^{(3)}+\tilde{p}_{L}^{(1)}-\tilde{p}_{L}^{(2)}\right)}\right]\right) \\
& +\frac{1}{\epsilon} \oint_{U} \frac{d u}{2 \pi}\left(\operatorname{Li}_{2}\left[e^{i\left(\hat{p}^{(3)}+\hat{p}^{(1)}+\hat{p}^{(2)}\right)}\right]-\operatorname{Li}_{2}\left[e^{i\left(\tilde{p}_{R}^{(3)}+\tilde{p}_{L}^{(1)}+\tilde{p}_{L}^{(2)}\right)}\right]\right) .
\end{aligned}
$$

As is clear from the above expressions, $\left[\mathcal{C}_{123}^{\bullet \bullet \bullet}\right]^{\text {asympt }}$ precisely matches the result of our analysis (2.7) and (2.8). Furthermore, when restricting to the one non-BPS and two BPS correlators, we can see that the first term in $\left[\mathcal{C}_{123}^{\bullet \bullet}\right]^{\text {wrapping }}$ coincides with our result of the resummation of the bottom mirror particles $\left[\mathcal{C}_{123}^{\bullet \circ}\right]^{\text {bottom }}$ in (2.10) and (6.41). Similar match can be seen also in the $\mathfrak{s l}(2)$ sector, where the result from the string theory reads

$$
\begin{aligned}
& \log \left[\mathcal{C}_{123}^{\bullet \bullet \bullet}\right]_{\mathfrak{s l}(2)}^{\text {asympt }}=\frac{1}{\epsilon} \oint_{\mathcal{C}_{\mathbf{u}_{1} \cup \mathbf{u}_{2}}} \frac{d u}{2 \pi} \operatorname{Li}_{2}\left[e^{\left.i \hat{p}_{L}^{(1)}+i \hat{p}_{L}^{(2)}-i \hat{p}_{R}^{(3)}\right]}+\frac{1}{\epsilon} \oint_{\mathcal{C}_{\mathbf{u}_{3}}} \frac{d u}{2 \pi} \operatorname{Li}_{2}\left[e^{i \hat{p}_{R}^{(3)}+i \hat{p}_{L}^{(2)}-i \hat{p}_{L}^{(1)}}\right]\right. \\
& \log \left[\mathcal{C}_{123}^{\bullet \bullet \bullet \bullet ~}\right]_{\mathfrak{s l}(2)}^{\text {wrapping }}= \frac{1}{\epsilon} \oint_{U} \frac{d u}{2 \pi}\left(\operatorname{Li}_{2}\left[e^{i\left(\hat{p}_{L}^{(1)}+\hat{p}_{L}^{(2)}-\hat{p}_{R}^{(3)}\right)}\right]-\operatorname{Li}_{2}\left[e^{i\left(\tilde{p}^{(1)}+\tilde{p}^{(2)}-\tilde{p}^{(3)}\right)}\right]\right) \\
&+\frac{1}{\epsilon} \oint_{U} \frac{d u}{2 \pi}\left(\operatorname{Li}_{2}\left[e^{i\left(\hat{p}_{L}^{(2)}+\hat{p}_{R}^{(3)}-\hat{p}_{L}^{(1)}\right)}\right]-\operatorname{Li}_{2}\left[e^{i\left(\tilde{p}^{(2)}+\tilde{p}^{(3)}-\tilde{p}^{(1)}\right)}\right]\right) \\
&+\frac{1}{\epsilon} \oint_{U} \frac{d u}{2 \pi}\left(\operatorname{Li}_{2}\left[e^{i\left(\hat{p}_{R}^{(3)}+\hat{p}_{L}^{(1)}-\hat{p}_{L}^{(2)}\right)}\right]-\operatorname{Li}_{2}\left[e^{i\left(\tilde{p}^{(3)}+\tilde{p}^{(1)}-\tilde{p}^{(2)}\right)}\right]\right) \\
&+\frac{1}{\epsilon} \oint_{U} \frac{d u}{2 \pi}\left(\operatorname{Li}_{2}\left[e^{i\left(\hat{p}_{R}^{(3)}+\hat{p}_{L}^{(1)}+\hat{p}_{L}^{(2)}\right)}\right]-\operatorname{Li}_{2}\left[e^{i\left(\tilde{p}^{(3)}+\tilde{p}^{(1)}+\tilde{p}^{(2)}\right)}\right]\right) .
\end{aligned}
$$

The remaining factors in $\left[\mathcal{C}_{123}^{\bullet \bullet \bullet}\right]^{\text {wrapping }}$ supposedly come from other mirror channels. It would be an important future problem to reproduce those remaining terms by resumming the mirror particles in other channels.

\section{Asymptotic structure constant for two BPS and one non-BPS operator}

In this section we are computing the structure constant for the case of a single non-BPS operator. Although this can be considered as a particular case of the one treated in the

\footnotetext{
${ }^{4}$ In the convention of this paper.
} 
next section, we prefer to work out in detail the clustering method on the simpler case, and then have a result ready to use for to the more complicated case. Since the $\mathfrak{s u}(2)$ and $\mathfrak{s l}(2)$ sectors are largely similar, we treat only the former in detail, and just give the results and point out the main difference for the latter.

\subsection{From sum-over-partition to multiple contour integral}

In the definition of the structure constant, the three operators are represented by on-shell states of three different spin chains of lengths $L_{1}, L_{2}, L_{3}$. Only the first chain of length $L \equiv L_{1}$ has non-trivial excitations (magnons) with momenta $p_{1}, \ldots, p_{M}, M \equiv M_{1}$. The momenta are parametrised by the corresponding rapidities $\mathbf{u}=\left\{u_{1}, \ldots, u_{M}\right\}$ according to

$$
e^{i p(u)}=\frac{x(u+i \epsilon / 2)}{x(u-i \epsilon / 2)} .
$$

Above, we have rescaled the rapidity variables by $\epsilon$ which will be set at the typical value for the rapidities $\mathbf{u}$. In the regime dual to semiclassical strings, this overall scale is $\epsilon \sim 1 / L_{1}$. The semiclassical limit is $\epsilon \rightarrow 0$. The Zhukovsky variable $x(u)$ is defined as

$$
x(u)=\frac{u+\sqrt{u^{2}-(2 g \epsilon)^{2}}}{2 g \epsilon} .
$$

The rapidities $\mathbf{u}$ satisfy the Bethe equations

$$
e^{i \phi_{j}}=1, \quad j=1, \ldots, M
$$

where $\phi_{j}$ is the total scattering phase for the $j$-th magnon

$$
e^{\phi_{j}}=e^{-i p\left(u_{j}\right) L} \prod_{k(\neq j)} S\left(u_{j}, u_{k}\right)
$$

$S(u, v)$ being the scattering matrix, which can be represented as the ratio

$$
S(u, v)=\frac{h(v, u)}{h(u, v)} .
$$

The function $h(u, v)$, which is given in our case by $h(u, v)_{\mathfrak{s u}(2)} \equiv h_{Y Y}(u, v)$, is the building block for the hexagon expansion in the configuration described above. It is given by the product of three factors,

$$
h(u, v)_{\mathfrak{s u}(2)}=\frac{u-v}{u-v+i \epsilon} \frac{1}{s(u, v) \sigma(u, v)},
$$


where $s(u, v)$ is the symmetric part,

$$
s(u, v)=\frac{\left(1-1 / x^{+} y^{+}\right)\left(1-1 / x^{-} y^{-}\right)}{\left(1-1 / x^{+} y^{-}\right)\left(1-1 / x^{-} y^{+}\right)}
$$

and $\sigma(u, v)=1 / \sigma(v, u)$ is the square root of the BES dressing phase [32, 33]. The reason to split $h(u, v)$ as above is that at tree $(g=0)$ level, $s(u, v)=\sigma(u, v)=1$. It will be important in the following that neither $s(u, v)$ nor $\sigma(u, v)$ has singularities close to $u=v$. We use the notation $x^{ \pm}=x(u \pm i \epsilon / 2)$ and $y^{ \pm}=x(v \pm i \epsilon / 2)$. The unnormalised structure constant, is defined as a sum over partitions of the rapidities $\mathbf{u}$ into two subsets, $\mathbf{u}=\alpha \cup \bar{\alpha}$,

$$
\left[\mathcal{C}_{123}^{\bullet \circ \circ}\right]^{\text {asympt }} \equiv \mathscr{A}=\sum_{\alpha \cup \bar{\alpha}=\mathbf{u}}(-1)^{|\bar{\alpha}|} \prod_{j \in \alpha} e^{i p\left(u_{j}\right) \ell_{R}} \prod_{j \in \alpha, k \in \bar{\alpha}} \frac{1}{h\left(u_{k}, u_{j}\right)},
$$

where $\ell_{R}=\frac{1}{2}\left(L_{1}+L_{3}-L_{2}\right)$ is the length of the bridge between the first operator (on the top) and the third one. In order to have a complete match with the original tree-level result reported in $[6,24]$, we will work with an equivalent representation,

$$
\mathscr{A}=\sum_{\alpha \cup \bar{\alpha}=\mathbf{u}}(-1)^{|\alpha|} \prod_{j \in \alpha} e^{\left.-i p\left(u_{j}\right)\right) \ell} \prod_{j \in \alpha, k \in \bar{\alpha}} \frac{1}{h\left(u_{j}, u_{k}\right)}, \quad \ell \equiv \ell_{L},
$$

where $\ell_{L}=\frac{1}{2}\left(L_{2}+L_{1}-L_{3}\right)$ is the length of the bridge connecting the first and the second operator. The equivalence of the two expression can be shown by using the Bethe ansatz equations (3.3) with $L_{1}=\ell_{L}+\ell_{R}$. Formally, at tree-level, the two expressions (3.9) and (3.8) can be obtained from each other by exchanging $\ell_{L}$ and $\ell_{R}$ and sending $\epsilon \rightarrow-\epsilon$.

Extending the tree-level observation in [9], the sum over partitions (3.9) can be written as a multiple contour integral

$$
\mathscr{A}=\sum_{n=0}^{N} \frac{1}{n !} \oint_{\mathcal{C}_{\mathbf{u}}} \prod_{j=1}^{n} \frac{d z_{j}}{2 \pi \epsilon} F\left(z_{j}\right) \prod_{j<k}^{n} h\left(z_{j}, z_{k}\right) h\left(z_{k}, z_{j}\right),
$$

where the integration contour $\mathcal{C}_{\mathbf{u}}$ closely encircles the rapidities $\mathbf{u}=\left\{u_{1} \ldots u_{N}\right\}$ counterclockwise, the function $F(x)$ is given by

$$
F(z)=\frac{e^{-i p(z) \ell} \mu(z)}{h(z, \mathbf{u})}, \quad h(z, \mathbf{u}) \equiv \prod_{j=1}^{N} h\left(z, u_{j}\right)
$$

and the measure

$$
\mu(z)=\frac{\left(1-1 / x^{+} x^{-}\right)^{2}}{\left(1-1 /\left(x^{+}\right)^{2}\right)\left(1-1 /\left(x^{-}\right)^{2}\right)}
$$

is defined so that $h(z, u) \simeq i \epsilon \mu(z)(z-u)$ at $z=u$. 
In the all loop pairwise interaction

$$
\Delta^{\text {all }}\left(z_{j}, z_{k}\right) \equiv h\left(z_{j}, z_{k}\right) h\left(z_{k}, z_{j}\right)=\Delta\left(z_{j}, z_{k}\right) s\left(z_{j}, z_{k}\right)^{2}
$$

the dressing factor drops out due to the anti-symmetry of the dressing phase. In the semiclassical limit $\epsilon \rightarrow 0$, the deviation of the interaction $\Delta^{\text {all }}(u, v)$ with respect with its tree level value $\Delta(u, v)$ is subleading,

$$
\Delta^{\text {all }}(u, v)=\Delta(u, v)\left(1-c(u, v, g \epsilon)^{2} \epsilon^{2}+\mathcal{O}\left(\epsilon^{3}\right)\right)
$$

where $c(u, v, g \epsilon)$ is some function of the rapidities $u$ and $v$ and the effective coupling $g^{\prime}=$ $g \epsilon$. It is important that even at strong coupling, where $g^{\prime}$ is finite, the correction to the interaction is subleading. A similar property is valid for the measure $\mu(u)$

$$
\mu(u)=1-c(u, u, g \epsilon) \epsilon^{2}+\mathcal{O}\left(\epsilon^{3}\right)
$$

This will allow us to take the semiclassical limit of the asymptotic contribution for any value of the coupling constant, including strong coupling. The main steps of the derivation can be understood on the tree-level example, which can be treated exactly and will be worked out in detail in the following. The clustering procedure explained below works exactly as in the tree-level, as long as the integration contours are kept as distance from the cuts of the dressing phase $\sigma(u, v)$, that is out of the region $-2 g^{\prime}<\operatorname{Re}\left(z_{k}\right)<2 g^{\prime}$. This is certainly the case for semiclassical strings.

\subsection{Tree-level revisited}

The structure constant of one non-BPS and two BPS operators at three level $\mathscr{A}$ was first studied thoroughly in [24] and [6]. In this section, we revisit the tree-level result by a different method which allows direct generalisations to all loops.

The starting point is the multiple integral contour integral (3.10)

$$
\mathscr{A}=\sum_{n=0}^{N} \frac{1}{n !} \oint_{\mathcal{C}_{\mathbf{u}}} \prod_{j=1}^{n} \frac{d z_{j}}{2 \pi \epsilon} F\left(z_{j}\right) \prod_{j<k}^{n} \Delta\left(z_{j}, z_{k}\right) \text {, }
$$

where the different ingredients take their tree-level values ${ }^{5}$

$$
F(z)=\frac{e^{-i p(z) \ell}}{h(z, \mathbf{u})}, \quad h(z, u)=\frac{z-u}{z-u+i \epsilon} .
$$

\footnotetext{
${ }^{5}$ To avoid proliferation of symbols we keep the same notations as for all-loop case for most objects.
} 
The sum (3.16) is given by single integrals coupled by the pairwise interaction $\Delta(u, v)$ defined as

$$
\begin{aligned}
\Delta(u, v) & =h(u, v) h(v, u)=\frac{(u-v)^{2}}{(u-v)^{2}+\epsilon^{2}} \\
& =1+\frac{i \epsilon / 2}{u-v-i \epsilon}-\frac{i \epsilon / 2}{u-v+i \epsilon}
\end{aligned}
$$

In other words, the function $\Delta(u, v)$ differs from 1 only when $|u-v| \sim \epsilon$, and it has two poles at $u=v \pm i \epsilon$. For later convenience, we define a generalisation of this function

$$
\begin{aligned}
\Delta_{m n}(u, v) & =\frac{(u-v)(u-v+i(m-n) \epsilon)}{(u-v+i m \epsilon)(u-v-i n \epsilon)} \\
& =1-\frac{m n}{m+n}\left(\frac{i \epsilon}{u-v+i m \epsilon}+\frac{i \epsilon}{u-v-i n \epsilon}\right)
\end{aligned}
$$

so that $\Delta(u, v)=\Delta_{1,1}(u, v)$. The summation limit in (3.16) can be extended to infinity, since the result of integration is zero if there are more integrals than rapidities in the set $\mathbf{u}$. The multiple contour integral representation (3.16) is our starting point. Similar integrals have appeared recently in the context of integrable probabilities for example in $[16,17]$ and [18].

Semiclassical limit. The rhs of (3.16) can be viewed as a grand canonical partition function of a matrix model. This matrix model appeared when computing the partition function of dimensionally reduced SYM with four supercharges [34]. The semiclassical limit (large $N$ or large chemical potential for the grand canonical partition function) was found in [35] using the standard matrix model techniques. The spectral curve of the matrix model is associated with an elliptic Riemann surface with two parallel cuts at distance $\epsilon$ from each other.

The semiclassical limit we are interested in is more subtle. It consists of taking the limit $L, M \rightarrow \infty$ so that $M / L \sim 1$, or taking $\epsilon \rightarrow 0$ so that $M \epsilon$ remains finite. ${ }^{6}$ In this limit, which is very similar to the Nekrasov-Shatashvili limit [13], the standard matrix model techniques do not work. The leading and the subleading term of the partition function were evaluated in [9] by representing the partition function as a Fredholm determinant and resolving the corresponding Riemann-Hilbert problem. A shorter, although less rigorous derivation used the mapping to a system of chiral fermions.

\footnotetext{
${ }^{6}$ This is the large $L$ limit of a solution of the Bethe equations, characterised by one or more Bethe strings with mode numbers $n_{k}$ and filling fractions $\alpha_{k}=M_{k} / L_{1}$. When $L_{1} \rightarrow \infty$, the distribution of the magnon rapidities along each Bethe string converges to a continuous linear density. This limit of the spin chain has been first studied in [36] and then rediscovered in the context of AdS/CFT in [37].
} 
Here we will give a rigorous derivation of the semiclassical limit based on an exact evaluation of each term in the sum in (3.16) and then taking the limit. We will observe a formation of bound states in close analogy to the bound states of instantons appearing in the Nekrasov-Shatashvili limit.

\subsection{Deformation of contours and clustering}

Here we will set up a procedure which allows to perform an expansion in the parameter $\epsilon$ around the semiclassical limit $\epsilon \rightarrow 0, L \sim M \sim 1 / \epsilon \rightarrow \infty$ of the functional $\mathscr{A}$ in (3.16). Namely, we deform the integration contours sequentially so that they become widely separated and far way from the support of $\mathbf{u}$, as is shown in figure 3.4. After the contour

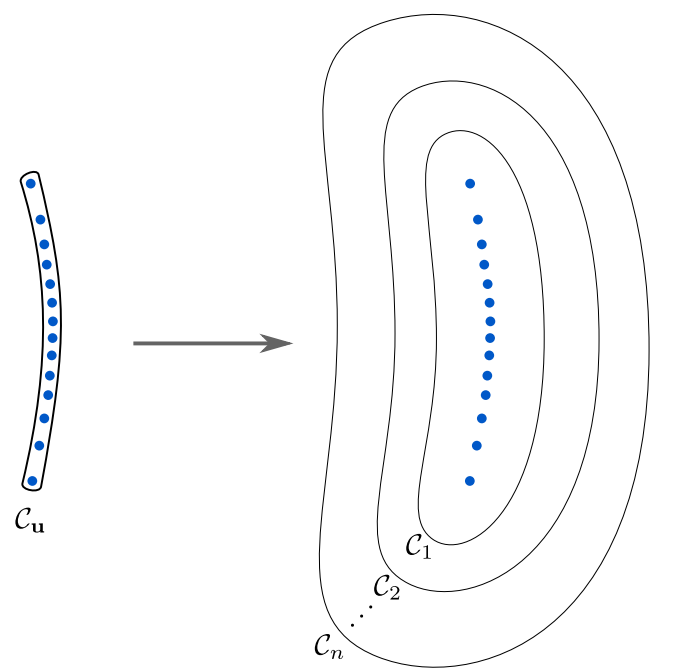

Figure 3.4: Deformation of the integration contours. Here $\mathcal{C}_{k}$ is the deformed contour of the integration variable $x_{k}$, which is situated at a distance larger than $\epsilon$ from all the other contours.

deformation, we have $\left|z_{j}-z_{k}\right| \gg \epsilon$ and the singularities in the multiple integrals are removed. In the procedure of deformation of contours, one has to take into account the residues of the poles in the interaction terms $\Delta\left(z_{i}, z_{j}\right)$ in $(3.16)$. This leads to a phenomenon we call clustering which was considered in various forms in [13, 34], in [14, 15] and in [16, 18] and which is reminiscent of the formation of bound states as solutions of the Bethe equations. A similar procedure was suggested in [10] in order to take the strong coupling limit of the scattering amplitudes for gluons. Let us consider in more detail an integral of the type

$$
\mathrm{I}_{n}=\oint_{\mathcal{C}_{\mathbf{u}}} \prod_{j=1}^{n} \frac{d z_{j}}{2 \pi \epsilon} F\left(z_{j}\right) \prod_{j<k}^{n} \Delta\left(z_{j}, z_{k}\right),
$$


which corresponds to the $n$-th term in the sum in (3.16). The integrand is a product of functions $F(z)$ and $\Delta\left(z_{i}, z_{j}\right)$. We can imagine a collection of $n$ particles, each particle $z_{i}$ is associated with a function $F\left(z_{i}\right)$ and between any two particles $z_{i}$ and $z_{j}$, there are interactions described by the function $\Delta\left(z_{i}, z_{j}\right)$. Then the integrand can be represented by the diagram shown in figure 3.5.

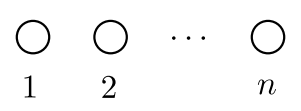

Figure 3.5: A diagrammatic representation of the integrand of $\mathrm{I}_{n}$.

In order to illustrate the idea, we analyse an example for $n=3$ explicitly. We start with

$$
\mathrm{I}_{3}=\oint_{\mathcal{C}_{\mathbf{u}}} \frac{d z_{1} d z_{2} d z_{3}}{(2 \pi \epsilon)^{3}} F\left(z_{1}\right) F\left(z_{2}\right) F\left(z_{3}\right) \Delta\left(z_{1}, z_{2}\right) \Delta\left(z_{1}, z_{3}\right) \Delta\left(z_{2}, z_{3}\right)
$$

We first deform the contour of integration for $z_{3}$ from $\mathcal{C}_{\mathbf{u}}$ to a contour $\mathcal{C}_{3}$ which is situated outside $\mathcal{C}_{\mathbf{u}}$ at a distance larger than $\epsilon$. There are poles at $z_{3}=z_{2} \pm i \epsilon$ and $z_{1} \pm i \epsilon$ due to the interaction $\Delta\left(z_{1}, z_{3}\right)$ and $\Delta\left(z_{2}, z_{3}\right)$, respectively. If we take the pole at $z_{3}=z_{2}-i \epsilon$, the residue is proportional to the following integral

$$
\oint_{\mathcal{C}_{\mathbf{u}}} \frac{d z_{1} d z_{2}}{(2 \pi \epsilon)^{2}} F\left(z_{1}\right) F\left(z_{2}\right) F\left(z_{2}-i \epsilon\right) \Delta\left(z_{1}, z_{2}\right) \Delta\left(z_{1}, z_{2}-i \epsilon\right) .
$$

Because

$$
F\left(z_{2}\right) F\left(z_{2}-i \epsilon\right)=\left(\frac{z_{2}-3 i \epsilon / 2}{z_{2}+i \epsilon / 2}\right)^{\ell} \frac{z_{2}-\mathbf{u}+i \epsilon}{z_{2}-\mathbf{u}-i \epsilon}
$$

is analytic inside the contour $\mathcal{C}_{\mathbf{u}}$, the integration over $z_{2}$ gives zero. The same argument works for $z_{3}=z_{1}-i \epsilon$. This implies that we only need to consider the poles $z_{3}=z_{2}+i \epsilon$ and $z_{3}=z_{1}+i \epsilon$. If we take the pole $z_{3}=z_{2}+i \epsilon$, the result reads

$$
\frac{1}{2} \oint_{\mathcal{C}_{\mathbf{u}}} \frac{d z_{1} d z_{2}}{(2 \pi \epsilon)^{2}} F\left(z_{1}\right) F\left(z_{2}\right) F\left(z_{2}+i \epsilon\right) \Delta\left(z_{1}, z_{2}\right) \Delta\left(z_{1}, z_{2}+i \epsilon\right) .
$$

We have taken here into account that, while deforming the counter-clockwise contour $\mathcal{C}_{\mathbf{u}}$ into $\mathcal{C}_{k}$, the contours surrounding the poles will be oriented clockwise. Let us define the functions $F_{1}, F_{2}, F_{3}$, etc. by

$$
F_{n}(z)=F(z) F(z+i \epsilon) \cdots F(z+(n-1) i \epsilon)
$$


Using the fact that

$$
\Delta(u, v) \Delta(u, v+i \epsilon)=\Delta_{1,2}(u, v)
$$

where $\Delta_{1,2}(u, v)$ is defined in (3.19), we can write the residue (3.24) as

$$
\frac{1}{2} \oint_{\mathcal{C}_{\mathbf{u}}} \frac{d z_{1} d z_{2}}{(2 \pi \epsilon)^{2}} F\left(z_{1}\right) F_{2}\left(z_{2}\right) \Delta_{1,2}\left(z_{1}, z_{2}\right) .
$$

This result can be interpreted as the following. Taking the residue gives rise to a cluster, or bound state, of length 2 . The function associated to this cluster is given by $F_{2}(z)$ and its interaction with a fundamental particle at the point $z^{\prime}$ is described by $\Delta_{1,2}\left(z, z^{\prime}\right)$. This is symbolised graphically in figure 3.6, left.

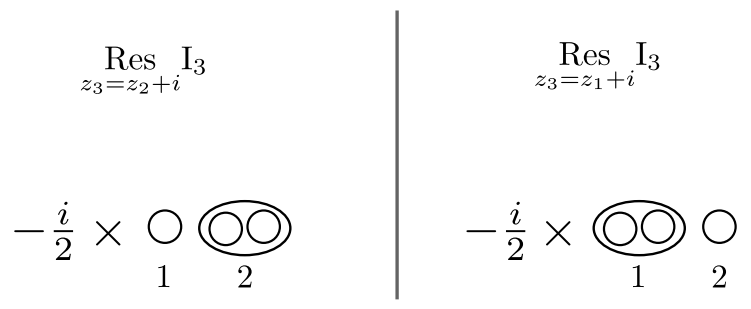

Figure 3.6: The clustering of fundamental particles into bound states.

When moving their integration contours from $\mathcal{C}_{\mathbf{u}}$ to $\mathcal{C}_{j}$, the bound states themselves undergo further clustering and form larger bound states. A length $n$ bound state is associated to the wave function $F_{n}(z)$ defined in (3.25) and the interaction between bound states of length $m$ and $n$ is described by $\Delta_{m n}(z, w)$. The full result of our example $n=3$, which illustrates the origin of the combinatorial factors, is given in appendix A. In terms of diagrams, it is given in figure 3.7 .

As we can see, the result is given by the sum of all possible bound states, each bound state of length $n$ multiplied by a factor $1 / n$. To see that this is true in general, let us consider the integral with a bound state of length $m$ and length $n$

$$
\oint_{\mathcal{C}_{\mathbf{u}}} \frac{d z_{j}}{2 \pi \epsilon} \frac{d z_{k}}{2 \pi \epsilon} \cdots \frac{F_{m}\left(z_{j}\right)}{m} \times \frac{F_{n}\left(z_{k}\right)}{n} \Delta_{m n}\left(z_{j}, z_{k}\right) \cdots
$$

Suppose we now want to deform the contour $z_{k}$ to $\mathcal{C}_{k}$ and pick the pole $z_{k}=z_{j}+i m \epsilon$. The 


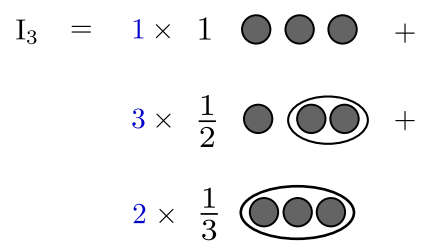

Figure 3.7: The final result of $I_{3}$ after deforming the contours. Here the black dots mean the integration contour for $x_{j}$ is $\mathcal{C}_{j}$. The numbers in blue represent the multiplicities of clusters and they are given by equation 3.35 , for example $C_{3}^{1,1,1}=1, C_{3}^{1,2}=3$ and $C_{3}^{3}=2$.

extra contribution from the pole is

$$
\begin{aligned}
& \oint_{\mathcal{C}_{\mathbf{u}}} \frac{d z_{j}}{2 \pi \epsilon} \cdots \frac{F_{m}\left(z_{j}\right)}{m} \times \frac{F_{n}\left(z_{j}+i m\right)}{n} \times\left(\frac{m n}{m+n}\right) \cdots \\
& =\oint_{\mathcal{C}_{\mathbf{u}}} \frac{d z_{j}}{2 \pi \epsilon} \cdots\left(\frac{F_{m+n}\left(z_{j}\right)}{m+n}\right)
\end{aligned}
$$

where we have used that

$$
F_{m}(z) F_{n}(z+i m)=F_{m+n}(z), \quad \operatorname{Res}_{v=u+i m \epsilon} \Delta_{m n}(v, u)=\frac{i m n \epsilon}{m+n} .
$$

In what follows we will denote the fusion rules like (3.29) simply as

$$
\frac{F_{m}\left(z_{j}\right)}{m} \times \frac{F_{n}\left(z_{j}+i m \epsilon\right)}{n} \rightarrow \frac{F_{m+n}\left(z_{j}\right)}{m+n} .
$$

The fusion rules ensure that the final result is a sum over all possible bound state configurations. Each configuration comes with a combinatorial factor. We will derive these factors and write down an exact expression for $\mathrm{I}_{n}$ in the next section.

\subsection{The exact result and semiclassical limit}

In this section, we give an exact expression for $\mathrm{I}_{n}$ and $\mathscr{A}$ and then take its semiclassical limit. As discussed above, while deforming the contour we need to pick up poles which lead to the formation of bound states. The final result is a sum over all possible configurations of bound states

$$
\mathrm{I}_{n}=\sum_{k=1}^{n} \sum_{q_{1}+\cdots q_{k}=n} C_{n}^{q_{1}, \cdots, q_{k}} \prod_{j=1}^{k} \oint_{\mathcal{C}_{j}} \frac{d z_{j}}{2 \pi \epsilon} \frac{F_{q_{j}}\left(z_{j}\right)}{q_{j}} \prod_{i<j}^{n} \Delta_{q_{i}, q_{j}}\left(z_{i}, z_{j}\right) .
$$

Here $k$ is the number of bound states in a given configuration and $q_{1} \leq \cdots \leq q_{k}$ are the lengths of the bound states. They should satisfy $q_{1}+\cdots+q_{k}=n . F_{q_{j}}\left(z_{j}\right)$ is the wavefunction 
for the bound state defined in (3.25) and $\Delta_{q_{i}, q_{j}}\left(z_{i}, z_{j}\right)$ is defined in (3.19). The combinatorial factor $C_{n}^{q_{1}, \cdots, q_{k}}$ counts the number of the bound state configuration with lengths $\left\{q_{1}, \cdots, q_{k}\right\}$. In what follows, it is convenient to represent the bound state configuration in a different way. Suppose among the bound state configurations $\left\{q_{1}, q_{2}, \cdots, q_{k}\right\}, d_{l}$ of them have length $l(l=1,2, \cdots)$, then we can represent the configuration by a vector $\vec{d}=\left\{d_{1}, d_{2}, \cdots\right\}$ :

$$
\left\{q_{1}, \cdots, q_{k}\right\}=\{\underbrace{1 \cdots 1}_{d_{1}}, \cdots, \underbrace{l, \cdots, l}_{d_{l}}, \cdots\} \mapsto \vec{d}=\left\{d_{1}, d_{2}, \cdots\right\} .
$$

We will use the two notations interchangeably. The following two obvious identities will be useful

$$
\sum_{j=1}^{k} F\left(q_{j}\right)=\sum_{l=1}^{\infty} d_{l} F(l), \quad \prod_{j=1}^{k} F\left(q_{j}\right)=\prod_{l=1}^{\infty} F(l)^{d_{l}} .
$$

In particular, the constraint $\sum_{j=1}^{k} q_{j}=n$ can be rewritten as $\sum_{l} d_{l} l=n$. We have

$$
\begin{aligned}
C_{n}^{q_{1}, \ldots, q_{k}} & =\frac{1}{d_{1} ! d_{2} ! \cdots}\left(\begin{array}{c}
n \\
q_{1}
\end{array}\right)\left(\begin{array}{c}
n-q_{1} \\
q_{2}
\end{array}\right) \cdots\left(\begin{array}{c}
q_{k} \\
q_{k}
\end{array}\right)\left(q_{1}-1\right) ! \cdots\left(q_{k}-1\right) ! \\
& =\frac{1}{d_{1} ! d_{2} ! \cdots} \frac{n !}{q_{1} \cdots q_{k}}=\frac{n !}{\prod_{l} l^{d_{l}} d_{l} !} .
\end{aligned}
$$

Let us explain briefly how to obtain the first line of the expression above. It is constituted from three different blocks: the middle one is the way to make $k$ clusters of lengths $n_{1} \leq$ $\ldots \leq n_{k}$ out of $n$ variables, while the first block insures that clusters with the same number of elements are indistinguishable. The last block gives the number of different ways to arrange the objects inside each cluster. For a cluster with $n_{1}$ elements, one can choose the label of the surviving integration variable at will, while the number of different possible orders of clustering for the other variables is $\left(n_{1}-1\right)$ !. Inserting (3.35) into (3.32) and summing over $n$ we obtain the exact result

$$
\begin{aligned}
\mathscr{A} & =\sum_{k} \sum_{q_{1} \leq \cdots \leq q_{k}} \frac{1}{d_{1} ! d_{2} ! \cdots} \prod_{j=1}^{k} \oint_{\mathcal{C}_{j}} \frac{d z_{j}}{2 \pi \epsilon} \frac{F_{q_{j}}\left(z_{j}\right)}{q_{j}^{2}} \prod_{i<j}^{k} \Delta_{q_{i}, q_{j}}\left(z_{i}, z_{j}\right) \\
& =\sum_{k} \frac{1}{k !} \sum_{q_{1}, \ldots, q_{k}} \prod_{j=1}^{k} \oint_{\mathcal{C}_{j}} \frac{d z_{j}}{2 \pi \epsilon} \frac{F_{q_{j}}\left(z_{j}\right)}{q_{j}^{2}} \prod_{i<j}^{k} \Delta_{q_{i}, q_{j}}\left(z_{i}, z_{j}\right) .
\end{aligned}
$$

In the last line the summation over $q_{j}$ is unrestricted. This exact expression can be taken as the starting point for a systematic semiclassical expansion. There are two sources of $\epsilon$ corrections, from the wavefunction $F_{n}(z)$ and from the interaction $\Delta_{m n}\left(z_{i}, z_{j}\right)$,

$$
\begin{aligned}
F_{n}(z) & =F(z)^{n}+\epsilon \frac{n(n-1)}{2} F(z)^{n-1} \partial_{z} F(z)+\mathcal{O}\left(\epsilon^{2}\right) \\
\Delta_{m n}\left(z_{i}, z_{j}\right) & =1-\frac{m n}{\left(z_{i}-z_{j}\right)^{2}} \epsilon^{2}+\mathcal{O}\left(\epsilon^{3}\right) .
\end{aligned}
$$


If we are interested in the leading order of $\epsilon$ expansion of (3.36) we can replace $F_{n}(z)$ by $F^{n}(z)$ and $\Delta_{m n}\left(z_{i}, z_{j}\right)$ by 1 , which simplifies (3.36) drastically. The multiple integrals decouple and the result exponentiates,

$$
\mathscr{A} \simeq \sum_{k} \frac{1}{k !} \prod_{j=1}^{k} \sum_{q_{j}} \oint_{\mathcal{C}_{j}} \frac{d z_{j}}{2 \pi \epsilon} \frac{F\left(z_{j}\right)^{q_{j}}}{q_{j}^{2}}=\exp \oint_{\mathcal{C}_{\mathbf{u}}} \frac{d z}{2 \pi \epsilon} \sum_{q} \frac{F(z)^{q}}{q^{2}} .
$$

Here the integration contour is far way from the support of $\mathbf{u}$, but now we can deform it back to encircle closely the support of the rapidities $\mathbf{u}$. We recognise in the expression above the expansion of the dilogarithm. Taking into account the subleading corrections from (3.37) we obtain the first two terms from [9]

$$
\log \mathscr{A}=\oint_{\mathcal{C}_{\mathbf{u}}} \frac{d z}{2 \pi \epsilon} \operatorname{Li}_{2}[F(z)]-\frac{1}{2} \oint_{\mathcal{C}_{\mathbf{u}}^{\times 2}} \frac{d z d z^{\prime}}{(2 \pi)^{2}} \frac{\log [1-F(z)] \log \left[1-F\left(z^{\prime}\right)\right]}{\left(z-z^{\prime}\right)^{2}}+\ldots
$$

To avoid the singularity when $z$ and $z^{\prime}$ coincide in the double contour integration above, the two contours can be separated, which is equivalent to taking the principal value integral. More terms in the expansion (3.39) can in principle be obtained by a cluster expansion of (3.36).

\subsection{The semiclassical limit for the $\mathfrak{s u}(2)$ sector}

We now specialise the expression in (3.39) to the particular case of the $\mathfrak{s u}(2)$ sector

$$
\log \mathscr{A} \simeq \oint_{\mathcal{C}_{\mathbf{u}}} \frac{d z}{2 \pi \epsilon} \operatorname{Li}_{2}\left[e^{-i p(z) \ell+i G_{\mathbf{u}}(z)}\right]
$$

which agrees with the results in [6] and [8]. Above, we denoted with $p(z)$ and $G_{\mathbf{u}}(z)$ the momentum and resolvent at tree level, in the semiclassical limit $\epsilon \rightarrow 0$,

$$
p^{\text {tree }}(z)=\frac{\epsilon}{z}, \quad G_{\mathbf{u}}^{\text {tree }}(z)=\sum_{i=1}^{N} \frac{\epsilon}{z-u_{i}} .
$$

The all-loop result has exactly the same structure, but with the quasi-momentum replaced by its full expression, which contains now the dressing phase,

$$
G_{\mathbf{u}}(z)=\sum_{i=1}^{N}\left[\frac{\epsilon}{z-u_{i}}-i \log \sigma\left(z, u_{i}\right)\right] .
$$

In the physical regime the dressing phase can be expressed as

$$
-i \log \sigma(u, v)=\chi\left(u^{+}, v^{-}\right)+\chi\left(u^{-}, v^{+}\right)-\chi\left(u^{+}, v^{+}\right)-\chi\left(u^{-}, v^{-}\right) \simeq \epsilon^{2} \partial_{u} \partial_{v} \chi(u, v),
$$


with $\chi(u, v)$ given by an integral representation [38]. Defining the sphere all-loop quasimomenta $\tilde{p}^{(k)}(z)$ by

$$
\tilde{p}^{(2,3)}(z)=\epsilon \frac{x^{\prime}(z) L_{2,3}}{2 x(z)}, \quad \tilde{p}^{(1)}(z)=\epsilon \frac{x^{\prime}(z) L_{1}}{2 x(z)}-G_{\mathbf{u}}(z),
$$

the semiclassical limit of the asymptotic all-order contribution is given by

$$
\log \mathscr{A}=\oint_{\mathcal{C}_{\mathbf{u}}} \frac{d z}{2 \pi \epsilon} \operatorname{Li}_{2}\left[e^{i\left(\tilde{p}^{(3)}(z)-\tilde{p}^{(2)}(z)-\tilde{p}^{(1)}(z)\right)}\right]=-\oint_{\mathcal{C}_{\mathbf{u}}} \frac{d z}{2 \pi \epsilon} \operatorname{Li}_{2}\left[e^{i\left(\tilde{p}^{(3)}(z)-\tilde{p}^{(2)}(z)+\tilde{p}^{(1)}(z)\right)}\right]
$$

The last expression is the semiclassical limit of (3.9). We used that

$$
\left(e^{-i \tilde{p}^{(1)}(z)}\right)_{\text {on the first sheet }}=\left(e^{i \tilde{p}^{(1)}(z)}\right)_{\text {on the second sheet }}
$$

which is a consequence of the classical limit of the Bethe equations (3.4),

$$
\tilde{p}(u+i 0)+\tilde{p}(u-i 0)=L p(u)-\left(G_{\mathbf{u}}(u+i 0)+G_{\mathbf{u}}(u-i 0)\right)=0 \bmod (2 \pi)
$$

and that the contour of integration changes its orientation when deformed to the second sheet.

In the strong coupling limit the dressing phase simplifies, $\chi(u, v) \simeq \frac{u-v}{\epsilon} \log \left(1-\frac{1}{x y}\right)$. Since $\frac{1}{u-v}=-\partial_{u} \partial_{v}(u-v) \log (u-v)$ the resolvent becomes

$$
G_{\mathbf{u}}(z)=\epsilon \sum_{i=1}^{N} \frac{x^{\prime}\left(u_{i}\right)}{x(z)-x\left(u_{i}\right)}-p(x) \epsilon \sum_{i=1}^{N} \frac{x^{\prime}\left(u_{i}\right)}{x^{2}\left(u_{i}\right)} \equiv \mathcal{G}_{\mathbf{u}}(x)-\frac{\Delta-L}{2} p(x),
$$

with $\Delta-L$ the anomalous dimension, or the spin-chain energy. The quasi-momenta $\tilde{p}^{(k)}(z)$ assume in this limit the simpler form

$$
\tilde{p}^{(2,3)}(z)=\epsilon \frac{x^{\prime}(z) L_{2,3}}{2 x(z)}, \quad \tilde{p}^{(1)}(z)=\epsilon \frac{x^{\prime}(z) \Delta}{2 x(z)}-\mathcal{G}_{\mathbf{u}}(x(z)) .
$$

\subsection{The semiclassical limit for the $\mathfrak{s l}(2)$ sector}

The expression for the three-point function with a single non-BPS operator in the $\mathfrak{s l}(2)$ sector is the same as $(3.9)$ with $h(u, v)=h_{\mathfrak{s u}(2)}(u, v)$ replaced with the corresponding $\mathfrak{s l}(2)$ quantity

$$
h_{\mathfrak{s}(2)}(u, v)=\frac{x^{+}-y^{-}}{x^{-}-y^{+}} h_{\mathfrak{s u}(2)}(u, v)=\frac{u-v}{u-v-i \epsilon} \frac{1-1 / x^{-} y^{+}}{1-1 / x^{-} y^{-}} \frac{1-1 / x^{-} y^{+}}{1-1 / x^{+} y^{+}} \frac{1}{\sigma(u, v)} .
$$


At tree-level, $\mathscr{A}_{\mathfrak{s l}(2)}$ can be obtained from $\mathscr{A}_{\mathfrak{s u}(2)}$ just by sending $\epsilon \rightarrow-\epsilon$ and $\ell \rightarrow-\ell$. At higher loop, the change comes from changing the expression of $h(u, v)$ as in (3.50), which affects the expression of the quasi-momenta in the semiclassical limit,

$$
f_{\mathfrak{s l}(2)}(u) \rightarrow e^{i\left(\hat{p}^{(3)}(z)-\hat{p}^{(2)}(z)-\hat{p}^{(1)}(z)\right)} .
$$

The quasi-momenta appearing in the asymptotic part of the $\mathfrak{s l}(2)$ structure constant correspond now to the AdS part of the spectral curve [39, 40],

$$
\hat{p}^{(2,3)}(z)=\epsilon \frac{x^{\prime}(z) L_{2,3}}{2 x(z)}, \quad \hat{p}^{(1)}(z)=\epsilon \frac{x^{\prime}(z) L_{1}}{2 x(z)}+\mathcal{G}_{\mathbf{u}}(x(z)) .
$$

The slightly different appearance of (3.52) with respect to (3.49) is due to the extra factor in the second member of (3.50).

We can therefore write the semiclassical limit of the asymptotic all-order contribution in the $\mathfrak{s l}(2)$ sector as

$$
\log \mathscr{A}_{\mathfrak{s l}(2)}=-\oint_{\mathcal{C}_{\mathbf{u}}} \frac{d z}{2 \pi \epsilon} \operatorname{Li}_{2}\left[e^{i\left(\hat{p}^{(3)}(z)-\hat{p}^{(2)}(z)-\hat{p}^{(1)}(z)\right)}\right]=\oint_{\mathcal{C}_{\mathbf{u}}} \frac{d z}{2 \pi \epsilon} \operatorname{Li}_{2}\left[e^{i\left(\hat{p}^{(3)}(z)-\hat{p}^{(2)}(z)+\hat{p}^{(1)}(z)\right)}\right] .
$$

Upon permutation of indices 2 and 3, which is possible due to symmetry, this expression coincides with the strong coupling result (2.14).

\section{Asymptotic structure constant for three non-BPS fields}

Here we consider the all-loop prediction for a configuration equivalent to that studied in [24] where two of the operators belong to the left sector and the third operator belongs to the right sector of $\mathfrak{s o}(4)=\mathfrak{s u}(2)_{L} \oplus \mathfrak{s u}(2)_{R}$. The excitations for the three operators are chosen to be the longitudinal scalars

$$
\begin{aligned}
& \mathcal{O}_{1} \in \mathfrak{s u}(2)_{L}: \text { vacuum } Z^{L_{1}}, \quad M_{1} \text { excitations } Y=\Phi_{1 \dot{2}} \text {, } \\
& \mathcal{O}_{2} \in \mathfrak{s u}(2)_{L}: \operatorname{vacuum} Z^{L_{2}}, \quad M_{2} \text { excitations } Y=\Phi_{1 \dot{2}} \text {, } \\
& \mathcal{O}_{3} \in \mathfrak{s u}(2)_{R}: \text { vacuum } Z^{L_{3}}, \quad M_{3} \text { excitations } \bar{Y}=\Phi_{21} \text {. }
\end{aligned}
$$

After the twisted rotation the three operators are mapped to operators type $\{Z, Y\}$ at the origin, $\{\bar{Z}, \bar{Y}\}$ at infinity, and $\{\tilde{Z}, \tilde{Y}\}$ at some finite point, say $\vec{x}=(0,0,1,0)$, where

$\tilde{Z}=\frac{1}{2}(Z+Y+\bar{Z}-\bar{Y}), \quad \tilde{Y}=\frac{1}{\sqrt{2}}(\bar{Y}-\bar{Z})$. This corresponds, in the conventions of [1], to excitations $\chi_{\text {top }}=\chi_{\text {bottom }}=\chi_{\text {reservoir }}=Y$. 
To compute such three-point functions using the hexagon, we first collect all the scalar excitations to one of the edges by performing the mirror transformation $\gamma$ several times [1]. (See figure 2.1 for the configuration of the excitations before performing the mirror transformations.) After collecting them on the second edge $\left(\mathcal{O}_{2}\right)$ on the left hexagon and on the first edge $\left(\mathcal{O}_{1}\right)$ on the right hexagon, we obtain the hexagons with $\left\{\alpha_{1}^{4 \gamma}, \alpha_{3}^{2 \gamma}, \alpha_{2}\right\}$ and with $\left\{\bar{\alpha}_{2}^{4 \gamma}, \bar{\alpha}_{3}^{2 \gamma}, \bar{\alpha}_{1}\right\}$. There are of course several other ways to collect the excitations to one of the edges. However, the advantage of the choice described here is that all the excitations become $Y$ after the transformation owing to the transformation property of the excitations clarified in [1]:

$$
Y \stackrel{2 \gamma}{\rightarrow}-\bar{Y}, \quad \bar{Y} \stackrel{2 \gamma}{\rightarrow}-Y
$$

Then, since all the excitations are of the $Y$ type, the hexagon form factor factorises into two-particle form factors ${ }^{7} h(u, v)$.

We also study an analogous configuration in the $\mathfrak{s l}(2)$ sector, where $\mathcal{O}_{1}$ and $\mathcal{O}_{2}$ contain $D$ excitations and $\mathcal{O}_{3}$ contains $\bar{D}$ excitations. The hexagon form factor for this configuration can be computed in a similar way, namely by collecting all the excitations to the one of the edges by using the mirror transformations.

\subsection{Formulation in terms of multiple contour integrals}

The asymptotic part of the un-normalised structure constant with three non-BPS operators,

which we denote by $\left[\mathcal{C}_{123}^{\bullet \bullet \bullet}\right]$, is given by a sum over the partitions of all the three sets of Bethe roots into left and right subsets, $\mathbf{u}_{i}=\alpha_{i} \cup \bar{\alpha}_{i}$ :

$$
\begin{aligned}
{\left[\mathcal{C}_{123}^{\bullet \bullet \bullet}\right] } & =\sum_{\alpha_{i} \cup \bar{\alpha}_{i}=\mathbf{u}^{(i)}} \prod_{i=1}^{3}(-1)^{\left|\alpha_{1}\right|+\left|\alpha_{2}\right|+\left|\alpha_{3}\right|} w_{\ell_{31}}\left(\alpha_{1}, \bar{\alpha}_{1}\right) w_{\ell_{12}}\left(\alpha_{2}, \bar{\alpha}_{2}\right) w_{\ell_{23}}\left(\alpha_{3}, \bar{\alpha}_{3}\right) \\
& \times \quad \mathrm{H}\left(\alpha_{1}\left|\alpha_{3}\right| \alpha_{2}\right) \mathrm{H}\left(\bar{\alpha}_{2}\left|\bar{\alpha}_{3}\right| \bar{\alpha}_{1}\right)
\end{aligned}
$$

with the splitting factors given by

$$
w_{\ell}(\alpha, \bar{\alpha})=e^{-i p_{\alpha} \ell} \frac{h^{<}(\bar{\alpha}, \alpha)}{h^{>}(\alpha, \bar{\alpha})}, \quad h^{\gtrless}(\mathbf{u}, \mathbf{v}) \equiv \prod_{\substack{j<k \\ j<k}} h\left(u_{j}, v_{k}\right) .
$$

\footnotetext{
${ }^{7}$ Here we included the matrix part $A(u, v)$ is included in the definition of $h(u, v)$ for the $\mathfrak{s u}(2)$ sector, as we did in section 3 .
} 
The hexagon form factor can be computed by performing crossing transformation on all the excitations to bring them on the same edge

$$
\begin{aligned}
& \mathrm{H}\left(\alpha_{1}\left|\alpha_{3}\right| \alpha_{2}\right)=\text { phase }_{1} \mathrm{H}\left(\alpha_{1}^{4 \gamma} ; \alpha_{3}^{2 \gamma} ; \alpha_{2}\right) \\
& \mathrm{H}\left(\bar{\alpha}_{2}\left|\bar{\alpha}_{3}\right| \bar{\alpha}_{1}\right)=\text { phase }_{2} \mathrm{H}\left(\bar{\alpha}_{2}^{4 \gamma} ; \bar{\alpha}_{3}^{2 \gamma} ; \bar{\alpha}_{1}\right) .
\end{aligned}
$$

A subtle point is the definition of the crossing-transformed factors. For fields from the $\mathfrak{s l}(2)$ sector it is sufficient to change the argument $x^{ \pm} \rightarrow 1 / x^{ \pm}$. In the general case the crossing transformation is more complicated. It is computed by going to string frame, perform the analytic continuation and transforming back to the spin frame, cf. appendix $\mathrm{F}$ of [1]. In general the hexagon form factor contains a matrix and a scalar part, cf. equation (2) of [1].

As we mentioned above, in the $\mathfrak{s l}(2)$ case the matrix part of the hexagon form factors is trivial and the weights in the sum over partitions are products of scalar factors:

$$
\begin{aligned}
{\left[\mathcal{C}_{123}^{\bullet \bullet \bullet}\right]^{\text {asympt }}=} & \sum_{\alpha_{i} \cup \bar{\alpha}_{i}=\mathbf{u}_{i}}(-1)^{\left|\alpha_{1}\right|+\left|\alpha_{3}\right|+\left|\alpha_{3}\right|} e^{-i p\left(\alpha_{1}\right) \ell_{31}} e^{-i p\left(\alpha_{2}\right) \ell_{12}} e^{-i p\left(\alpha_{3}\right) \ell_{23}} \\
& \frac{h\left(\alpha_{1}^{4 \gamma}, \alpha_{2}\right) h\left(\alpha_{1}^{4 \gamma}, \alpha_{3}^{2 \gamma}\right) h\left(\alpha_{3}^{2 \gamma}, \alpha_{2}\right) h\left(\bar{\alpha}_{2}^{4 \gamma}, \bar{\alpha}_{1}\right) h\left(\bar{\alpha}_{2}^{4 \gamma}, \bar{\alpha}_{3}^{2 \gamma}\right) h\left(\bar{\alpha}_{3}^{2 \gamma}, \bar{\alpha}_{1}\right)}{h\left(\alpha_{1}, \bar{\alpha}_{1}\right) h\left(\alpha_{2}, \bar{\alpha}_{2}\right) h\left(\alpha_{3}, \bar{\alpha}_{3}\right)} \times \text { phase }
\end{aligned}
$$

For fields from the $\mathfrak{s l}(2)$ sector the crossing transformation is done analytically continuing $x^{ \pm} \rightarrow 1 / x^{ \pm}$and phase $=1$. For $\mathfrak{s u}(2)$ fields the phase factors are derived in in Appendix C. The explicit forms of the hexagon amplitudes in the two sectors is given in (3.50). and the factors $h\left(u^{4 \gamma}, v\right)$ and $h\left(u^{2 \gamma}, v^{2 \gamma}\right)$ are related to $h(u, v)$ in a simple way:

$$
h\left(u^{4 \gamma}, v\right)=1 / h(v, u), \quad h\left(u^{2 \gamma}, v^{2 \gamma}\right)= \begin{cases}h(u, v) & \text { for } \mathfrak{s l}(2), \\ h(u, v) e^{i p(u)-i p(v)} & \text { for } \mathfrak{s u}(2) .\end{cases}
$$

The unnormalised structure constant takes the same form for $\mathfrak{s u}(2)$ and $\mathfrak{s l}(2)$ if we define

$$
b(u, v)=\left\{\begin{array}{ll}
h\left(u^{2 \gamma}, v\right)=\sigma(u, v) / A(u, v) & \text { for } \mathfrak{s l}(2), \\
e^{-i p(v)} h\left(u^{2 \gamma}, v\right)=\sigma(u, v) & \text { for } \mathfrak{s u}(2),
\end{array} \quad \text { with } \quad A(u, v)=\frac{1-\frac{1}{x^{-} y^{+}}}{1-\frac{1}{x^{+} y^{-}}} .\right.
$$

so that

$$
\left[\mathcal{C}_{123}^{\bullet \bullet \bullet}\right]^{\text {asympt }}=\sum_{\alpha_{i} \cup \bar{\alpha}_{i}=\mathbf{u}_{i}} \prod_{i=1}^{3}(-1)^{\left|\bar{\alpha}_{i}\right|} \frac{e^{-p\left(\alpha_{i}\right) \ell_{i-1, i}}}{h\left(\alpha_{i}, \bar{\alpha}_{i}\right)} \times \frac{1}{h\left(\alpha_{2}, \alpha_{1}\right) h\left(\bar{\alpha}_{1}, \bar{\alpha}_{2}\right)} \frac{b\left(\alpha_{3}, \alpha_{2}\right)}{b\left(\alpha_{3}, \alpha_{1}\right)} \frac{b\left(\overline{\alpha_{3}}, \bar{\alpha}_{1}\right)}{b\left(\bar{\alpha}_{3}, \bar{\alpha}_{2}\right)} .
$$

The next step is to convert the sum over partitions to a multiple contour integral, a 
generalisation of (3.10):

$$
\begin{gathered}
{\left[\mathcal{C}_{123}^{\text {•.• }}\right]^{\text {asympt }} \propto \sum_{m, n, r}^{\infty} \frac{1}{m ! n ! r !} \oint_{\mathcal{C}_{\mathbf{u}_{1}}} \prod_{j=1}^{m} \frac{\mu\left(z_{1, j}\right) d z_{1, j}}{2 \pi \epsilon} \oint_{\mathcal{C}_{\mathbf{u}_{2}}} \prod_{k=1}^{n} \frac{\mu\left(z_{2, k}\right) d z_{2, k}}{2 \pi \epsilon} \oint_{\mathcal{C}_{\mathbf{u}_{3}}} \prod_{l=1}^{r} \frac{\mu\left(z_{3, l}\right) d z_{3, l}}{2 \pi \epsilon}} \\
\times \frac{h^{\neq}\left(\mathbf{z}_{1}, \mathbf{z}_{1}\right)}{h\left(\mathbf{z}_{1}, \mathbf{u}_{1}\right)} \times \frac{h\left(\mathbf{z}_{1}, \mathbf{u}_{2}\right)}{b\left(\mathbf{u}_{3}, \mathbf{z}_{1}\right)} e^{-i p\left(\mathbf{z}_{1}\right) \ell_{13}} \\
\times \frac{h^{\neq}\left(\mathbf{z}_{2}, \mathbf{z}_{2}\right)}{h\left(\mathbf{z}_{2}, \mathbf{u}_{2}\right)} \times \frac{h\left(\mathbf{u}, \mathbf{z}_{2}\right)}{b\left(\mathbf{z}_{2}, \mathbf{u}_{3}\right)} e^{-i p\left(\mathbf{z}_{\mathbf{2}}\right) \ell_{12}} \\
\times \frac{h^{\neq}\left(\mathbf{z}_{3}, \mathbf{z}_{3}\right)}{h\left(\mathbf{z}_{3}, \mathbf{u}_{3}\right)} \times \frac{1}{b\left(\mathbf{u}_{2}, \mathbf{z}_{3}\right) b\left(\mathbf{z}_{3}, \mathbf{u}\right)} e^{-i p\left(\mathbf{z}_{3}\right) \ell_{23}} \\
\times \frac{b\left(\mathbf{z}_{1}, \mathbf{z}_{3}\right) b\left(\mathbf{z}_{3}, \mathbf{z}_{1}\right) b\left(\mathbf{z}_{2}, \mathbf{z}_{3}\right) b\left(\mathbf{z}_{3}, \mathbf{z}_{2}\right)}{h\left(\mathbf{z}_{1}, \mathbf{z}_{2}\right) h\left(\mathbf{z}_{2}, \mathbf{z}_{1}\right)}
\end{gathered}
$$

where the last line describes the interactions between different sets of variables $\mathbf{z}_{1}$ and $\mathbf{z}_{2}$. The numerator in the last line is equal to one due to the property $b(u, v) b(v, u)=1$, and thus the integration over the third set of variables $\mathbf{z}_{3}$ completely decouples. This is what is expected, since the left and the right $\mathfrak{s u}(2)$ fields do not feel each other perturbatively.

The integral (4.9) splits into three independent integrals of the type already studied in [9], if it were not for the bi-local factor entangling the groups $\mathbf{z}_{1}$ and $\mathbf{z}_{2}$ of variables. Remarkably, in the semiclassical limit $\epsilon \rightarrow 0$ and $\ell \epsilon$ finite, the integration contours for the variables $\mathbf{z}_{1}$ and $\mathbf{z}_{2}$ are at macroscopic distance and $h\left(\mathbf{z}_{1}, \mathbf{z}_{2}\right) h\left(\mathbf{z}_{2}, \mathbf{z}_{1}\right)=1+o(\epsilon)$.

In conclusion, the asymptotic coupling constant is given in the semiclassical limit again by a product of determinants. This can be used to work out a systematic quasi-classical expansion, which is however out of the scope of this paper. Our goal here is to compute the leading term and compare it with the result obtained on the string theory side [5]. In the semiclassical limit the structure constant factorises as

$$
\left[\mathcal{C}_{123}^{\bullet \bullet \bullet}\right]^{\text {asympt }} \propto \mathscr{A}_{1} \times \mathscr{A}_{2} \times \mathscr{A}_{3}
$$

where the integrals $\mathscr{A}_{1}, \mathscr{A}_{2}$ and $\mathscr{A}_{3}$, defined as

$$
\begin{aligned}
& \mathscr{A}_{1}=\sum_{n=0}^{\infty} \frac{1}{n !} \oint_{\mathcal{C}_{\mathbf{u}_{1}}} \prod_{j=1}^{n} \frac{\mu\left(z_{j}\right) d z_{j}}{2 \pi \epsilon} \times \frac{h^{\neq}(\mathbf{z}, \mathbf{z})}{h\left(\mathbf{z}, \mathbf{u}_{1}\right)} \times \frac{h\left(\mathbf{z}, \mathbf{u}_{2}\right)}{b\left(\mathbf{u}_{3}, \mathbf{z}\right)} e^{-i p(\mathbf{z}) \ell_{13}} \\
& \mathscr{A}_{2}=\sum_{n=0}^{\infty} \frac{1}{n !} \oint_{\mathcal{C}_{\mathbf{u}_{2}}} \prod_{j=1}^{n} \frac{\mu\left(z_{j}\right) d z_{j}}{2 \pi \epsilon} \times \frac{h^{\neq}(\mathbf{z}, \mathbf{z})}{h\left(\mathbf{z}, \mathbf{u}_{2}\right)} \times \frac{h\left(\mathbf{u}_{1}, \mathbf{z}\right)}{b\left(\mathbf{z}, \mathbf{u}_{3}\right)} e^{-i p(\mathbf{z}) \ell_{12}} \\
& \mathscr{A}_{3}=\sum_{n=0}^{\infty} \frac{1}{n !} \oint_{\mathcal{C}_{\mathbf{u}_{3}}} \prod_{j=1}^{n} \frac{\mu\left(z_{j}\right) d z_{j}}{2 \pi \epsilon} \times \frac{h^{\neq}(\mathbf{z}, \mathbf{z})}{h\left(\mathbf{z}, \mathbf{u}_{3}\right)} \times \frac{1}{b\left(\mathbf{u}_{2}, \mathbf{z}\right) b\left(\mathbf{z}, \mathbf{u}_{1}\right)} e^{-i p(\mathbf{z}) \ell_{23}}
\end{aligned}
$$


Neglecting the subleading factors in the product of the scalar factors, we can approximate the functionals $\mathscr{A}_{k}$ by the objects we have already computed in the previous section,

$$
\mathscr{A}_{k} \propto \sum_{n=0}^{\infty} \frac{1}{n !} \oint_{\mathcal{C}_{\mathbf{u}_{k}}} \prod_{j=1}^{n} \frac{d z_{j} \Lambda_{k}\left(z_{j}\right)}{2 \pi i} \prod_{i<j}^{n} \Delta\left(z_{i}, z_{j}\right)
$$

where the functions $\Lambda_{1}, \Lambda_{2}, \Lambda_{3}$ assemble the local factors for the three groups of integration variables:

$$
\begin{gathered}
\Lambda_{1}(z)=\frac{e^{-i \ell_{31} p(z)} h\left(z, \mathbf{u}_{2}\right)}{h\left(z, \mathbf{u}_{1}\right) b\left(\mathbf{u}_{3}, z\right)}, \quad \Lambda_{2}(z)=\frac{e^{-i \ell_{12} p(z)} h\left(\mathbf{u}_{1}, z\right)}{h\left(z, \mathbf{u}_{2}\right) b\left(z, \mathbf{u}_{3}\right)} \\
\Lambda_{3}(z)=\frac{e^{-i \ell_{23} p(z)}}{h\left(z, \mathbf{u}_{3}\right) b\left(\mathbf{u}_{2}, z\right) b\left(z, \mathbf{u}_{1}\right)}
\end{gathered}
$$

The three factors in the product (4.10) are exponentially small and the exponent of the product is given by

$$
\log \left[\mathcal{C}_{123}^{\bullet \bullet \bullet}\right]^{\text {asympt }}=\frac{1}{\epsilon}\left(\mathscr{Y}_{1}+\mathscr{Y}_{2}+\mathscr{Y}_{3}+o(\epsilon)\right) .
$$

where $\mathscr{Y}$ is a contour integral of a dilogarithm

$$
\mathscr{Y}_{i}= \pm \oint_{\mathcal{C}_{\mathbf{u}_{i}}} \frac{d u}{2 \pi} \operatorname{Li}_{2}\left(\Lambda_{i}(u)\right), \quad i=1,2,3 .
$$

where the $(+) \operatorname{sign}$ is for $\mathfrak{s u}(2)$ and the $(-) \operatorname{sign}$ is for $\mathfrak{s l}(2)$.

\subsection{Taking the semiclassical limit, $\mathfrak{s u}(2)$}

To obtain explicit expressions, we will express the local factors $\Lambda_{a}(u)$ in terms of the three quasi-momenta (4.19). Consider first the $\mathfrak{s u}(2)$ case where $b(u, v)=\sigma(u, v)$. In the leading order in $\epsilon$ we have (see Appendix B)

$$
\begin{aligned}
& \log h(u, v) \rightarrow-\frac{i \epsilon y^{\prime}}{x-y}+\frac{i p(x)}{y^{2}-1}=i \frac{\epsilon x^{\prime}}{y-x}-i \frac{p(y)}{x^{2}-1} \\
& \log b(u, v) \rightarrow-\frac{i \epsilon y^{\prime}}{1 / x-y}-\frac{i p(x)}{y^{2}-1}-i p(y)=\frac{i \epsilon x^{\prime}}{1 / y-x}+\frac{i p(y)}{x^{2}-1}+i p(x)
\end{aligned}
$$

or, after taking the product with $x_{j}=x\left(u_{j}\right), u_{j} \in \mathbf{u}$

$$
\begin{aligned}
& \log h(u, \mathbf{u}) \rightarrow-i \mathcal{G}_{\mathbf{u}}(x)+i \frac{\Delta-L}{2} p(x) \\
& \log b(u, \mathbf{u}) \rightarrow-i \mathcal{G}_{\mathbf{u}}(1 / x)-i \frac{\Delta-L}{2} p(x) \\
& \log h(\mathbf{u}, u) \rightarrow i \mathcal{G}_{\mathbf{u}}(x)-i \frac{\Delta-L}{2} p(x) \\
& \log b(\mathbf{u}, u) \rightarrow i \mathcal{G}_{\mathbf{u}}(1 / x)+i \frac{\Delta-L}{2} p(x),
\end{aligned}
$$


where the resolvent for the set $\mathbf{u}$ is defined $b y^{8}$

$$
\mathcal{G}_{\mathbf{u}}(x)=\sum_{j} \frac{x_{j}^{\prime}}{x-x_{j}}, \quad \sum_{j} \frac{1}{x_{j}^{2}-1}=\frac{\Delta-L}{2} .
$$

The next step is to express the measure factors (4.13) in terms of the quasi-momenta of the three operators

$$
\begin{aligned}
& \tilde{p}^{(1)}(x)=\frac{1}{2} \Delta_{1} p(x)-\mathcal{G}_{\mathbf{u}_{1}}(x), \\
& \tilde{p}^{(2)}(x)=\frac{1}{2} \Delta_{2} p(x)-\mathcal{G}_{\mathbf{u}_{2}}(x), \\
& \tilde{p}^{(3)}(x)=\frac{1}{2} \Delta_{3} p(x)-\mathcal{G}_{\mathbf{u}_{3}}(x) .
\end{aligned}
$$

Substituting (4.17) in (4.13) we get

$$
\begin{aligned}
& \Lambda_{1}(x) \rightarrow \exp \left(+i \tilde{p}^{(2)}(x)-i \tilde{p}^{(1)}(x)+i \tilde{p}^{(3)}(1 / x)\right) \\
& \Lambda_{2}(z) \rightarrow \exp \left(-i \tilde{p}^{(1)}(x)-i \tilde{p}^{(2)}(x)-i \tilde{p}^{(3)}(1 / x)\right) \\
& \Lambda_{3}(z) \rightarrow \exp \left(-i \tilde{p}^{(3)}(x)-i \tilde{p}^{(1)}(1 / x)+i \tilde{p}^{(2)}(1 / x)\right)
\end{aligned}
$$

Using the classical Bethe equations on the cut of $\tilde{p}^{(1)}$, we can change the sign of $\tilde{p}^{(1)}$ in the exponent and write $\mathscr{Y}_{1}$, eq. (4.15), as

$$
\mathscr{Y}_{1}=-\oint_{\mathcal{C}_{\mathbf{u}_{1}}} \frac{d u}{2 \pi} \operatorname{Li}_{2}\left(e^{i \tilde{p}^{(1)}(x)+i \tilde{p}^{(2)}(x)+i \tilde{p}^{(3)}(1 / x)}\right) .
$$

Here we took into account that the contour of integration changes its orientation when deformed to the second sheet. We also change the sign of the exponents in the other two integrals using the functional equation for the dilogarithm, $\operatorname{Li}_{2}\left(X^{-1}\right)=-\mathrm{Li}_{2}(X)-\frac{\pi^{2}}{6}-$ $\frac{1}{2} \log ^{2}(-X)$. This again leads to a minus sign in front of the integrals:

$$
\begin{aligned}
& \mathscr{Y}_{2}=-\oint_{\mathcal{C}_{\mathbf{u}_{2}}} \frac{d u}{2 \pi} \operatorname{Li}_{2}\left(e^{i \tilde{p}^{(1)}(x)+i \tilde{p}^{(2)}(x)+i \tilde{p}^{(3)}(1 / x)}\right) \\
& \mathscr{Y}_{3}=-\oint_{\mathcal{C}_{\mathbf{u}_{3}}} \frac{d u}{2 \pi} \operatorname{Li}_{2}\left(e^{-i \tilde{p}^{(2)}(1 / x)+i \tilde{p}^{(1)}(1 / x)+i \tilde{p}^{(3)}(x)}\right) .
\end{aligned}
$$

The final formula is

$$
\begin{aligned}
\log \left[\mathcal{C}_{123}^{\bullet \bullet \bullet \bullet}\right]_{\mathfrak{s u}(2)}^{\text {asympt }} & =-\frac{1}{\epsilon} \oint_{\mathcal{C}_{\mathbf{u}_{1} \cup \mathbf{u}_{2}}} \frac{d u}{2 \pi} \operatorname{Li}_{2}\left(e^{i \tilde{p}^{(1)}(x)+i \tilde{p}^{(2)}(x)+i \tilde{p}^{(3)}(1 / x)}\right) \\
& -\frac{1}{\epsilon} \oint_{\mathcal{C}_{\mathbf{u}_{3}}} \frac{d u}{2 \pi} \operatorname{Li}_{2}\left(e^{-i \tilde{p}^{(3)}(1 / x)+i \tilde{p}^{(2)}(x)-i \tilde{p}^{(1)}(x)}\right)+\text { subleading in } \epsilon .
\end{aligned}
$$

\footnotetext{
${ }^{8}$ We have set the mode numbers to zero for simplicity.
} 
This expression gives, up to subleading $o\left(\epsilon^{0}\right)$ terms, the exponent for the all-loop perturbative structure constant for three heavy fields.

Let us interpret this expression from the point of view of the spectral curves of the three heavy states which is written in termes of the classical monodromy matrix

$$
\Omega(u)=\operatorname{Diag}\left(e^{i \hat{p}_{1}(u)}, e^{i \hat{p}_{2}(u)}, e^{i \hat{p}_{3}(u)}, e^{i \hat{p}_{4}(u)} \mid e^{i \hat{p}_{1}(u)}, e^{i \hat{p}_{2}(u)}, e^{i \hat{p}_{3}(u)}, e^{i \hat{p}_{4}(u)}\right) .
$$

The finite zone solutions in this sector are characterised by cuts between 1-4 and 2-3 sheets of the Riemann surface. The Bethe equations give boundary conditions on these cuts for the combinations $\tilde{p}_{L}=\frac{1}{2}\left(\tilde{p}_{1}-\tilde{p}_{4}\right)$ and $\tilde{p}_{R}=\frac{1}{2}\left(\tilde{p}_{2}-\tilde{p}_{3}\right)$, representing the quasi-momenta in the left and in the right $\mathfrak{s u}(2)$ sectors. The spectral curve of the $S O(4)$ sector is invariant under the inversion symmetry $x \leftrightarrow 1 / x$, which exchanges $\tilde{p}_{L}$ and $\tilde{p}_{R}$

$$
\tilde{p}_{R}(x)=-\tilde{p}_{L}(1 / x) .
$$

This allows to go from the four-sheeted Riemann surface in the $u$-parametrization to a two-sheet Riemann surface in the $x$-parametrization

$$
\tilde{p}_{R}(x)=-\left.\tilde{p}(1 / x)\right|_{|x|>1}, \quad \tilde{p}_{L}(x)=\left.\tilde{p}(x)\right|_{|x|>1} .
$$

In the notations $p_{L, R}(u)$ via (4.25), the unnormalised structure constant takes the form

$$
\begin{aligned}
\log \left[\mathcal{C}_{123}^{\bullet \bullet \bullet}\right]_{\mathfrak{s u}(2)}^{\text {asympt }} & =-\frac{1}{\epsilon} \oint_{\mathcal{C}_{\mathbf{u}_{1} \cup \mathbf{u}_{2}}} \frac{d u}{2 \pi} \operatorname{Li}_{2}\left(e^{i \tilde{p}_{L}^{(1)}+i \tilde{p}_{L}^{(2)}-i \tilde{p}_{R}^{(3)}}\right)-\frac{1}{\epsilon} \oint_{\mathcal{C}_{\mathbf{u}_{3}}} \frac{d u}{2 \pi} \operatorname{Li}_{2}\left(e^{i \tilde{p}_{R}^{(3)}+i \tilde{p}_{L}^{(2)}-i \tilde{p}_{L}^{(1)}}\right) \\
& + \text { subleading in } \epsilon .
\end{aligned}
$$

In the strong coupling limit this expression reproduces exactly the the result of the string theory computation, eq. (2.12).

\subsection{Taking the semiclassical limit, $\mathfrak{s l}(2)$}

In the case of $\mathfrak{s l}(2)$ fields the scalar factors $h(u, v)$ have asymptotics, $c f$. appendix B,

$$
\begin{aligned}
\log h_{\mathfrak{s l}(2)}(u, v) & \rightarrow \frac{i \epsilon y^{\prime}}{x-y}-\frac{i p(y)}{x^{2}-1}=\frac{i \epsilon x^{\prime}}{x-y}+\frac{i p(x)}{y^{2}-1} \\
\log b_{\mathfrak{s l}(2)}(u, v) & \rightarrow \frac{i \epsilon y^{\prime}}{1 / x-y}+\frac{i p(y)}{1-1 / x^{2}}=\frac{i \epsilon x^{\prime}}{x-1 / y}-\frac{i p(x)}{1-1 / y^{2}}
\end{aligned}
$$

which gives

$$
\begin{aligned}
\log h(u, \mathbf{u}) & \rightarrow i \mathcal{G}_{\mathbf{u}}(x), \\
\log h(\mathbf{u}, u) & \rightarrow-i \mathcal{G}_{\mathbf{u}}(1 / x) \\
\log b(u, \mathbf{u}) & \rightarrow i \mathcal{G}_{\mathbf{u}}(1 / x) \\
\log b(\mathbf{u}, u) & \rightarrow-i \mathcal{G}_{\mathbf{u}}(1 / x)
\end{aligned}
$$


Substituting in (4.13), we obtain

$$
\begin{aligned}
& \Lambda_{1}(x) \rightarrow \exp \left(+i \hat{p}^{(2)}(x)-i \hat{p}^{(1)}(x)+i \hat{p}^{(3)}(1 / x)\right) \\
& \Lambda_{2}(z) \rightarrow \exp \left(-i \hat{p}^{(1)}(x)-i \hat{p}^{(2)}(x)-i \hat{p}^{(3)}(1 / x)\right) \\
& \Lambda_{3}(z) \rightarrow \exp \left(-i \hat{p}^{(3)}(x)-i \hat{p}^{(1)}(1 / x)+i \hat{p}^{(2)}(1 / x)\right)
\end{aligned}
$$

where $\hat{p}^{(k)}$ are the $\mathfrak{s l}(2)$ quasi-momenta,

$$
\hat{p}^{(k)}(x)=\frac{1}{2} L_{k} p(x)+\mathcal{G}_{\mathbf{u}_{k}}(x), \quad k=1,2,3 .
$$

The rest is in complete analogy with the $\mathfrak{s u}(2)$ sector. Taking into account the opposite sign of the dilogarithm, we write it as

$$
\begin{aligned}
\log \left[\mathcal{C}_{123}^{\bullet \bullet \bullet}\right]_{\mathfrak{s l}(2)}^{\text {asympt }} & =\frac{1}{\epsilon} \oint_{\mathcal{C}_{\mathbf{u}_{1} \cup \mathbf{u}_{2}}} \frac{d u}{2 \pi} \operatorname{Li}_{2}\left[e^{i \hat{p}_{L}^{(1)}+i \hat{p}_{L}^{(2)}-i \hat{p}_{R}^{(3)}}\right]+\frac{1}{\epsilon} \oint_{\mathcal{C}_{\mathbf{u}_{3}}} \frac{d u}{2 \pi} \operatorname{Li}_{2}\left[e^{i \hat{p}_{R}^{(3)}+i \hat{p}_{L}^{(2)}-i \hat{p}_{L}^{(1)}}\right] \\
& + \text { subleading in } \epsilon .
\end{aligned}
$$

which is what is expected from the strong coupling result [21] in (2.14).

\section{Bottom mirror excitations}

The full result of the structure constant requires taking into account mirror excitations on all the three edges. The general expression is too complicated to be treated here; moreover, the interaction of mirror particles in crossed channels is affected by singularities which need careful regularisation. The simplest, tractable case of mirror contribution is that of the structure constant with only one non-BPS operatos, in the channel opposed to the on the opposite edge of the physical excitations, or bottom channel, as shown in figure 2.3. These mirror particles do not enter the sum over partitions and they do not interact with the other mirror excitations, so they can be factorised out and considered separately. Written schematically in terms of the fundamental excitations, the integrand is given by [25]

$$
\mu\left(\mathbf{w}^{\gamma}\right) e^{i p\left(\mathbf{w}^{\gamma}\right) \ell_{B}} T\left(\mathbf{w}^{\gamma}\right) h^{\neq}\left(\mathbf{w}^{\gamma}, \mathbf{w}^{\gamma}\right) h\left(\mathbf{u}, \mathbf{w}^{-3 \gamma}\right)
$$

with $\ell_{B}=\frac{1}{2}\left(L_{2}+L_{3}-L_{1}\right)$ the length of the bottom bridge of the correlator, opposed to the operator $\mathcal{O}_{1}$. The last factor can also be transformed to the same mirror dynamics by using (4.6), $h\left(\mathbf{u}, \mathbf{w}^{-3 \gamma}\right)=1 / h\left(\mathbf{w}^{\gamma}, \mathbf{u}\right)$. The mirror transformation $\gamma$ is defined as the analytical continuation through the branch cut of the variable $x^{+}$, namely $x^{+} \rightarrow 1 / x^{+}$, as shown in 5.8 .

In [1] the contribution of a single mirror particle was analysed, and shown to reproduce lowest order contribution of the expected strong coupling answer [5, 21]. The full mirror 
corrections involve all the bound states, and in the integrand (2.5) all the quantities should be replaced by their bound state counterparts. Here we are able to sum all the bound state contribution, in the strong coupling limit, and to retrieve part of the strong coupling result. This imply summing over all the configurations $\vec{n}=\left\{n_{1}, n_{2}, \ldots\right\}$, where $n_{a}$ is the number of bound states of a magnons,

$$
\left[\mathcal{C}^{\bullet \circ \circ}\right]^{\text {bottom }}=\sum_{\vec{n}} \frac{\mathrm{B}[\vec{n}]}{\prod_{a} n_{a} !} .
$$

The contribution of the configuration $\vec{n}$ is given by ${ }^{9}$

$$
\begin{aligned}
\mathrm{B}[\vec{n}]=(-1)^{n} & \int_{-\infty}^{\infty} \prod_{a} \prod_{j=1}^{n_{a}} \frac{d z_{j}^{a}}{2 \pi \epsilon} \mu_{a}^{\gamma}\left(z_{j}^{a}\right) g_{a}^{\gamma}\left(z_{j}^{a}\right) T_{a}^{\gamma}\left(z_{j}^{a}\right) \\
& \times \prod_{\substack{a \\
1 \leq i<j \leq n_{a}}} H_{a a}^{\gamma}\left(z_{i}^{a}, z_{j}^{a}\right) \prod_{\substack{a<b \\
1 \leq i \leq n_{a} \\
1 \leq j \leq n_{b}}} H_{a b}^{\gamma}\left(z_{i}^{a}, z_{j}^{b}\right), \quad n=\sum_{a} n_{a} a .
\end{aligned}
$$

The integration contour is along the real axis in the mirror regime shown in 5.8. The bi-local factors $H_{a b}^{\gamma}\left(z_{i}^{a}, z_{j}^{b}\right)$ coupling two bound states of length $a$ and $b$ are given by

$$
H_{a b}^{\gamma}(u, v) \equiv h_{a b}\left(u^{\gamma}, v^{\gamma}\right) h_{b a}\left(v^{\gamma}, u^{\gamma}\right)
$$

where $h_{a b}(u, v)$ is the bound state counterpart of $h(u, v)$ and is defined in (5.7). The functions $g_{a}^{\gamma}(u) \equiv g_{a}\left(u^{\gamma}\right)$ and $\mu_{a}^{\gamma}(u) \equiv \mu_{a}\left(u^{\gamma}\right)$ are mirror transforms respectively of the local weight factor $g_{a}(u)$ and the measure $\mu_{a}(u)$ defined as

$$
g_{a}(u)=\frac{e^{i p_{a}(u) \ell_{B}}}{h_{a, 1}(u, \mathbf{u})}, \quad \mu_{a}(u)=\frac{1}{a} \frac{\left(1-1 / x^{[-a]} x^{[+a]}\right)^{2}}{\left(1-1 / x^{[+a]} x^{[+a]}\right)\left(1-1 / x^{[-a]} x^{[-a]}\right)} .
$$

Throughout this chapter we are using the notation $x^{[k]}=x(u+i k \epsilon / 2)$. We want to take the semiclassical limit of (5.2) and (5.3), focusing on the strong coupling limit $g \rightarrow \infty$. Since the $\mathfrak{s u}(2)$ and the $\mathfrak{s l}(2)$ cases are treated in almost identical way, we will focus on the $\mathfrak{s u}(2)$ case and will briefly summarise the $\mathfrak{s l}(2)$ case at the end.

\subsection{Quantities for bound states at strong coupling}

In this section, we determine the strong coupling limit expressions of the various quantities in the integrand (5.3). The bound state counterparts can be obtained by fusing the corresponding fundamental quantities. Notice that when we perform strong coupling expansion,

\footnotetext{
${ }^{9}$ The $(-1)^{n}$ factor comes from the crossing transformation of the mirror magnons $\Phi^{a \dot{a}} \stackrel{2 \gamma}{\rightarrow}-\Phi^{\dot{a} a}$.
} 
there are different regimes in the complex $x$-plane. Since the integration contours for the rapidities $\mathbf{z}$ are situated on the real axis in the mirror dynamics, it is enough to analyse the strong coupling limit in this regime [32]. The near-flat-space regime, where $u$ is situated close to the singularities $x(u)= \pm 1$ is not relevant for this case, as we are concerned with semiclassical strings. We have mainly to check the mirror giant magnon regime $|x(u)|>1$ and the mirror BMN regime $|x(u)|=1^{10}$. The integrals over mirror particles contain the factor

$$
e^{-E_{a}(u) \ell_{B}} \sim \frac{1}{x^{2 \ell_{B}}}, \quad|x(u)|>1
$$

which strongly suppresses the contribution of the mirror giant magnon particles for large values of the bridge length $\ell_{B}$. We are therefore going to concentrate on the BMN mirror regime $|x|=1$.

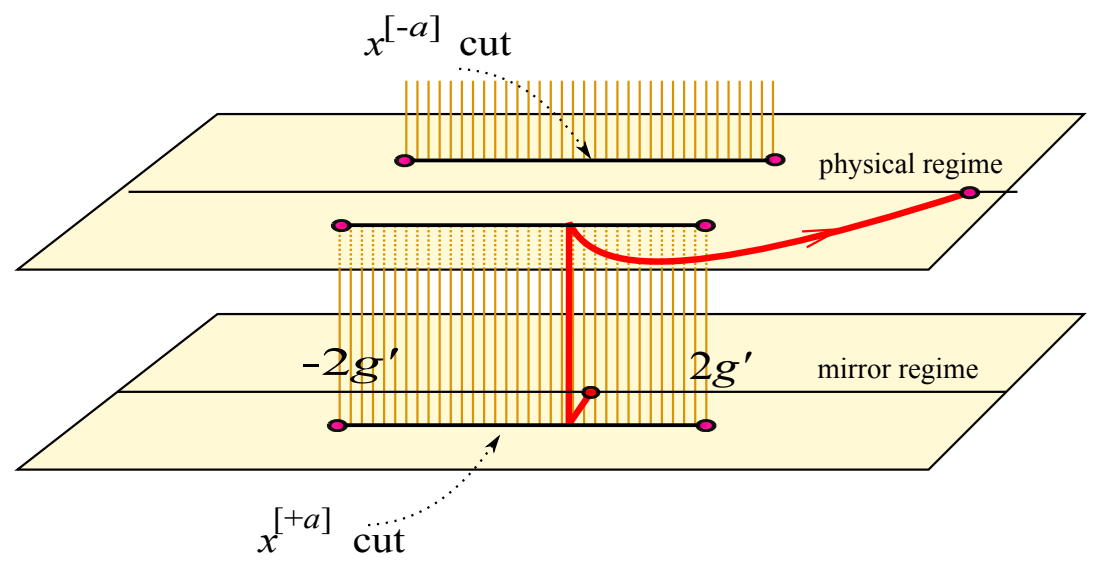

Figure 5.8: The rule for analytic continuation from the BMN mirror regime to BMN physical regime at strong coupling, when the real axis is pinched between the branch cuts of the Zhukovsky variables $x^{+}$and $x^{-}$.

As illustrated in 5.8, the contributions from the mirror BMN regime at strong coupling can be determined by first taking the strong-coupling limit of the relevant quantities in the physical regime and then analytically continuing them to the lower half of the unit circle $|x|=1$. This simple rule should be applied with care for the bound-state quantities, which may have an array of branch cuts. In this case, the passage to the mirror regime of an object associated to a bound state of size $a$ is done by substituting $x^{[+a]}(u)$ by $1 / x^{[+a]}(u)$, that is by analytically continuing $u$ through the branch cut of the Zhukovsky variable $x^{[+a]}(u)$ and leaving the other cut untouched.

\footnotetext{
10 The denomination of the various regimes follows the analytical continuation of the corresponding ones in the physical dynamics.
} 
In the strong coupling limit, the different branch cuts collapse on each other and on the real axis and the dependence on the rapidities will be given by variable with a single branch cut $x(u)$. In the mirror giant magnon regime, $x^{[+a]} \simeq x^{[-a]} \simeq x$, while in the mirror BMN regime, where the branch cut is situated, $x^{[+a]} \simeq 1 / x^{[-a]} \rightarrow x$. The net result is that after both mirror transformation and strong coupling limit, $x^{[ \pm a]} \rightarrow 1 / x$, which is equivalent to continuing $x$ to the lower half unit circle $U_{-}$.

Special care has to be devoted to the continuation of the dressing phase for bound states to the mirror dynamics, where extra cuts appear. As we explain in appendix D based on $[41,42]$, the dressing phase appears in combination with other functions which cancel exactly the cuts on the real axis and all the other cuts between those of $x^{[-a]}(u)$ and $x^{[+a]}(u)$. The same combination has no branch cut below that of $x^{[+a]}(u)$ in the physical dynamics, therefore we are again in the situation represented in figure 5.8 and we can use the analytical continuation from the BMN physical regime to the BMN mirror regime.

The scalar factor $H_{a b}(u, v)$. The scalar factor for scattering of two bound states of length $a$ and $b$ is given by

$$
h_{a b}(u, v)=\prod_{k=-\frac{a-1}{2}}^{\frac{a-1}{2}} \prod_{l=-\frac{b-1}{2}}^{\frac{b-1}{2}} h\left(u^{[2 k]}, v^{[2 l]}\right) .
$$

The symmetric scalar factor $H_{a b}(u, v)=h_{a b}(u, v) h_{b a}(v, u)$ is then given by

$$
H_{a b}(u, v)=\frac{x^{[-a]}-y^{[-b]}}{x^{[+a]}-y^{[-b]}} \frac{x^{[+a]}-y^{[+b]}}{x^{[-a]}-y^{[+b]}} \frac{1-1 / x^{[-a]} y^{[+b]}}{1-1 / x^{[+a]} y^{[+b]}} \frac{1-1 / x^{[+a]} y^{[-b]}}{1-1 / x^{[-a]} y^{[-b]}} .
$$

The dressing factor dropped out from the expression of the symmetric factor. In the strong coupling limit in the mirror dynamics $H^{\gamma}(u, v)$ takes the simple form

$$
H_{a b}^{\gamma}(u, v) \simeq \frac{u-v-i \epsilon \frac{a-b}{2}}{u-v-i \epsilon \frac{a+b}{2}} \quad \frac{u-v+i \epsilon \frac{a-b}{2}}{u-v+i \epsilon \frac{a+b}{2}} .
$$

We notice that the pairwise interaction takes in the strong coupling limit the same form as the interaction of the bound states (3.19) in the asymptotic structure constant,

$$
H_{a b}^{\gamma}(u, v) \simeq \Delta_{a b}\left(u^{[-a]}, v^{[-b]}\right)=\Delta_{a b}\left(u-\frac{1}{2} i a \epsilon, v-\frac{1}{2} i b \epsilon\right),
$$

the only difference being that the position of the pole is shifted to $v=u \pm i(a+b) \epsilon / 2$.

The measure $\mu_{a}(u)$. The expression for the measure for a bound state, eq.(5.5), is

$$
\mu_{a}(u)=\frac{1}{a} \frac{\left(1-1 / x^{[-a]} x^{[+a]}\right)^{2}}{\left(1-1 / x^{[+a]} x^{[+a]}\right)\left(1-1 / x^{[-a]} x^{[-a]}\right)} .
$$


Performing mirror transformation for $\mu_{a}(u)$ and expanding at strong coupling in the mirror BMN regime $|x|=1$, we find

$$
\mu_{a}\left(u^{\gamma}\right) \simeq \frac{1}{a}
$$

The factor $g_{a}(u)$. Recall that

$$
g_{a}(u)=\frac{e^{i p_{a}(u) \ell_{B}}}{h_{a 1}(u, \mathbf{u})}
$$

with

$$
h_{a 1}(u, v)=\frac{u^{[-a]}-v^{-}}{u^{[+a]}-v^{-}} \frac{1-1 / x^{[-a]} y^{+}}{1-1 / x^{[+a]} y^{+}} \frac{1-1 / x^{[+a]} y^{-}}{1-1 / x^{[-a]} y^{-}} \frac{1}{\sigma_{a, 1}} .
$$

After the continuation to the mirror dynamics, $\sigma_{a, 1}\left(u^{\gamma}, v\right)$ has extra cuts between those situated at $u-i a \epsilon / 2$ and $u+i a \epsilon / 2$ with $u \in\left[-2 g^{\prime}, 2 g^{\prime}\right]$. In particular, for even $a$ one of those cuts is situated on the real axis, i.e. on the contour of integration for the mirror particle contribution. These cuts are compensated by an extra factor coming from the normalisation of the transfer matrix matrix, as we will show below. The quantity we have to consider is

$$
\tilde{g}_{a}=g_{a}(u) \frac{R^{(-)[2-a]}}{R^{(+)[2-a]}} \cdots \frac{R^{(-)[a]}}{R^{(+)[a]}} .
$$

Here and below we use the notation

$$
\begin{aligned}
& R^{( \pm)}(u)=\left(x-\mathbf{x}^{\mp}\right) \equiv \prod_{j}\left(x(u)-x^{\mp}\left(u_{j}\right)\right) \\
& B^{( \pm)}(u)=\left(1 / x-\mathbf{x}^{\mp}\right) \equiv \prod_{j}\left(1 / x(u)-x^{\mp}\left(u_{j}\right)\right) .
\end{aligned}
$$

where the functions $R^{( \pm)}(u), B^{( \pm)}(u)$ play the role of the Baxter polynomials in the Zhukovsky plane and encode the rapidities of the incoming state. The simplest strategy to take the strong coupling limit of the quantity above is to compute it first in the BMN physical dynamics and the analytically continue to the BMN mirror dynamics. This can be done because there is no singularity to be met on the path of the analytical continuation. Taking the strong coupling limit, one can express $g_{a}(u)$ in terms of the three quasi-momenta (3.49)

$$
g_{a}(u) \rightarrow e^{i a p(x) \ell_{B}+i a \mathcal{G}(x)-i a \frac{\Delta-L_{1}}{2} p(x)}=e^{i a\left(\tilde{p}_{2}(x)+\tilde{p}_{3}(x)-\tilde{p}_{1}(x)\right)}
$$

and, after the continuation to the mirror regime one has

$$
\tilde{g}_{a}\left(u^{\gamma}\right)=\rightarrow e^{i a p(1 / x) \ell_{B}-i a \frac{\Delta-L_{1}}{2} p(1 / x)}=e^{i a\left(\tilde{p}_{2}(1 / x)+\tilde{p}_{3}(1 / x)-\tilde{p}_{1}(1 / x)\right)} e^{-i a \mathcal{G}(1 / x)} .
$$


We have used that at strong coupling we have, in the physical BMN regime

$$
f^{[a]}(u) \equiv \frac{R^{(+)[+a]}}{R^{(-)[+a]}} \rightarrow e^{i \mathcal{G}(x)}, \quad \bar{f}^{[a]}(u) \equiv \frac{B^{(-)[+a]}}{B^{(+)[+a]}} \rightarrow e^{-i \mathcal{G}(1 / x)}
$$

where

$$
\mathcal{G}(x)=\frac{1}{i} \sum_{j} \ln \frac{x-x_{j}^{-}}{x-x_{j}^{+}} \rightarrow \epsilon \sum_{j} \frac{x^{\prime}\left(u_{j}\right)}{x-x\left(u_{j}\right)}
$$

is the resolvent in the $x$-plane, while in the mirror BMN regime we have

$$
f^{[a]}\left(u^{\gamma}\right) \rightarrow e^{i \mathcal{G}(1 / x)}, \quad \bar{f}^{[a]}\left(u^{\gamma}\right) \rightarrow e^{-i \mathcal{G}(x)}
$$

The transfer matrix at strong coupling. Another important element for the integrand is the transfer matrix, arising after summing over the various polarisations of the mirror particles. The transfer matrix in the $\mathfrak{s u}(2)$ sector is given, up to a global factor, by $[26,43-46])$

$$
\begin{aligned}
& \sum_{a=0}^{\infty} \bar{T}_{a}^{[a-1]}(u) \mathrm{D}^{2 a}=\left(1-Y_{2,2} \mathrm{D}^{2}\right)\left(1-X_{2,1} \mathrm{D}^{2}\right)^{-1}\left(1-X_{1,1} \mathrm{D}^{2}\right)^{-1}\left(1-Y_{0,1} \mathrm{D}^{2}\right) \\
& \text { with } \quad X_{2,1}=X_{1,1}=1, \quad Y_{2,2}=\frac{R^{(-)}}{R^{(+)}}=\frac{1}{f^{-}}, \quad Y_{0,1}=\frac{B^{(+)+}}{B^{(-)+}}=\frac{1}{\bar{f}^{+}} \quad \text { and } \quad \mathrm{D}^{2}=e^{i \epsilon \partial_{u}}
\end{aligned}
$$

The bar on the function $T_{a}$ means complex conjugation, and assuming the rapidity $u$ to be real this means just changing the sign of the imaginary shifts.

A change in the normalisation of the transfer matrix can be obtained by multiplying the shift operator by an arbitrary function, $\mathrm{D}^{2} \rightarrow-\bar{N}(u) \mathrm{D}^{2}$ in the generating functional. Since we are using the $\mathfrak{s u}(2)$ hexagon form factor as the dynamical part, we should normalise the transfer matrices in such a way that the component in the $\mathfrak{s u}(2)$ sector is just 1 . This corresponds to taking $N(u)$ to be $R^{(-)+} / R^{(+)+}=1 / f^{+}$. Expanding (5.22) and taking into account the normalization, one obtains (see e.g. eq. (8.67) of [47])

$$
T_{a}(u)=(-1)^{a} N_{a}(u)\left[(a+1)-a \frac{R^{(+)[+a]}}{R^{(-)[+a]}}-a \frac{B^{(-)[-a]}}{B^{(+)[+a]}}+(a-1) \frac{R^{(+)[+a]}}{R^{(-)[+a]}} \frac{B^{(-)[-a]}}{B^{(+)[-a]}}\right]
$$

where $N_{a}(u)=N^{[1-a]} N^{[1-a+2]} \ldots N^{[a-1]}$. Notice that the bound-state quantities entering $g_{a}(u)$ in (5.5) have exactly the same structure as the prefactor $N_{a}(u)$. Therefore we can absorb $g_{a}(u)$ into the normalisation factor. This amounts to replacing $N_{a}(u)$ in $(5.23)$ with

$$
\tilde{g}_{a}(u) \equiv N_{a}(u) g_{a}(u) .
$$


We have prefered to keep the sign $(-1)^{a}$ out of the normalisation factor, since it will exactly compensate the factor $(-1)^{n}$ in (5.3). Thus the re-normalised transfer matrix $\mathrm{T}_{a}$ takes the form

$$
\mathrm{T}_{a}(u) \equiv g_{a}(u) T_{a}(u)=\tilde{g}_{a}(u)\left[(a+1)-a f^{[a]}-a \bar{f}^{[a]}+(a-1) f^{[a]} \bar{f}^{[a]}\right] .
$$

As it was discussed in the beginning of this chapter, the re-normalised transfer matrix $\mathrm{T}_{a}(u)$ does not have any cut beyond the cuts of $x^{[ \pm a]}$ on the physical sheet, and no cuts within the strip $|\Im u|<\frac{1}{2} a \epsilon$ on the mirror sheet. Therefore, the strong coupling limit of (5.25) in the BMN mirror dynamics can be obtained by simply substituting $x$ with $1 / x$ in the physical BMN expression,

$$
\mathrm{T}_{a}\left(u^{\gamma}\right) \rightarrow \tilde{g}^{a}\left(u^{\gamma}\right)[(a+1)-a f-a \bar{f}+(a-1) f \bar{f}],
$$

with $f$ and $\bar{f}$ being those from (5.21). Let us further define a quantity

$$
\mathrm{t}_{n}=\tilde{g}^{n}\left(2-f^{n}-\bar{f}^{n}\right) .
$$

It is interesting that only these quantities will appear in the final result of semiclassical limit. They can be expressed in terms of the transfer matrix by the following relations ${ }^{11}$

$$
\begin{aligned}
& \mathrm{t}_{1}=\mathrm{T}_{1} \\
& \mathrm{t}_{2}=2 \mathrm{~T}_{2}-\mathrm{T}_{1}^{2} \\
& \mathrm{t}_{3}=3 \mathrm{~T}_{3}-3 \mathrm{~T}_{2} \mathrm{~T}_{1}+\mathrm{T}_{1}^{3} \\
& \mathrm{t}_{4}=4 \mathrm{~T}_{4}-4 \mathrm{~T}_{3} \mathrm{~T}_{1}-2 \mathrm{~T}_{2}^{2}+4 \mathrm{~T}_{2} \mathrm{~T}_{1}^{2}-\mathrm{T}_{1}^{4} \\
& \mathrm{t}_{5}=5 \mathrm{~T}_{1}-5 \mathrm{~T}_{4} \mathrm{~T}_{1}-5 \mathrm{~T}_{3} \mathrm{~T}_{2}+5 \mathrm{~T}_{3} \mathrm{~T}_{1}^{2}+5 \mathrm{~T}_{2}^{2} \mathrm{~T}_{1}-5 \mathrm{~T}_{2} \mathrm{~T}_{1}^{3}+\mathrm{T}_{1}^{5},
\end{aligned}
$$

which can be derived from the generating functionals,

$$
\begin{aligned}
\operatorname{Sdet}(1-z \mathrm{G})^{-1} & =\frac{\left(1-z y_{1}\right)\left(1-z y_{2}\right)}{\left(1-z x_{1}\right)\left(1-z x_{2}\right)}=\sum_{a} z^{a} \mathrm{~T}_{a} \\
\operatorname{Str}(1-z \mathrm{G})^{-1} & =z \frac{\mathrm{d}}{\mathrm{d} z} \log \operatorname{Sdet}(1-z \mathrm{G})^{-1}=\sum_{a} z^{a} \mathrm{t}_{a} .
\end{aligned}
$$

Here "Sdet" and "Str" denote super-determinant and super-trace respectively and G a supergroup element with eigenvalues $\left(x_{1}, x_{2} \mid y_{1}, y_{2}\right)=\tilde{g}(1,1 \mid f, \bar{f})$. By inserting the first equation of (5.29) into the second equation of (5.29) and comparing the coefficient of $z^{n}$, we obtain the relations $(5.28)$. In general, the result reads

$$
\frac{\mathrm{t}_{n}}{n}=\sum_{\vec{n}: \sum_{a} n_{a} a=n}(-1)^{k-1}(k-1) ! \prod_{a} \frac{\mathrm{T}_{a}^{n_{a}}}{n_{a} !}, \quad k \equiv \sum_{a} n_{a} .
$$

\footnotetext{
${ }^{11}$ The relevance of this type of relations to clustering of mirror bound states was pointed out to us by Benjamin Basso. These relations are valid in the semiclassical limit only, both in the BMN and mirror dynamics, therefore we have dropped the arguments which specify the dynamics.
} 


\subsection{Clustering the mirror particles}

We have now prepared all the ingredients necessary to take the strong coupling limit of the contribution of mirror particles. After substituting the strong coupling quantities in the BMN mirror regime evaluated in the previous chapter, we obtain

$$
\mathrm{B}_{s c}[\vec{n}]=\int_{\mathrm{U}_{-}} \prod_{a} \prod_{j=1}^{n_{a}} \frac{d z_{j}^{a}}{2 \pi a \epsilon} \mathrm{T}_{a}\left(z_{j}^{a}\right) \prod_{\substack{a \\ 1 \leq i<j \leq n_{a}}} \Delta_{a a}\left(z_{i}^{a}, z_{j}^{a}\right) \times \prod_{\substack{a<b \\ 1 \leq i \leq n_{a} \\ 1 \leq j \leq n_{b}}} \Delta_{a b}\left(z_{i}^{a}-\frac{1}{2} i a \epsilon, z_{j}^{b}-\frac{1}{2} i b \epsilon\right)
$$

Here $\mathrm{B}_{s c}[\vec{n}]$ stands for the strong coupling limit of $\mathrm{B}[\vec{n}]$ in (5.3). Since in the strong coupling limit $\mathrm{T}_{a}(z)$ has a single branch cut, the one of the Zhukovsky variable $x(z)$ situated on the real axis, one has to specify the contour of integration. By convention we denote by $x$ the determination $x(z+i 0)$ and by $1 / x$ the determination $x(z-i 0)$, when $z$ is real. In agreement with the argument on the analytical continuation we have employed in the previous chapter, the integrals in (5.31) run on the contour just below the Zhukovsky cut, $\mathrm{U}_{-}=\left[-2 g^{\prime}-i 0,2 g^{\prime}-i 0\right]$, where the determination of the Zhukovsky variable is $1 / x$. The resummation of (5.2) will employ a method closely related to the clustering method from the asymptotic case.

The mechanism of clustering for mirror particles. In the asymptotic case, the contour of integration is closely surrounding the roots $\mathbf{u}$. By deforming the contour, we pick up poles $x_{k}=x_{j}+i m \epsilon$ which leads to clustering and makes it possible to safely take the semiclassical limit. Here the situation is slightly different since the contour is along the real axis in the complex $z$-plane. The integrals are independent except for the factors $\Delta_{a b}$ which become important at $\left|z_{j}-z_{k}\right| \sim \epsilon$. At strong coupling, it is convenient to use as rescaling factor $\epsilon=1 / 2 g$. When $g \rightarrow \infty$, the two poles of $\Delta_{a b}$ are approaching each other and pinch the integral contours on the real axis ${ }^{12}$. In order to avoid this singularity, we can deform the contours. Again the deformation of contours will catch poles and leads to clustering. Here we are interested in obtaining only the limit $g \rightarrow \infty$ and not the $1 / g$ corrections, which are more involved and which are left for a future work. A more straightforward, equivalent way to obtain the result is to notice that

$$
\Delta_{a b}\left(z_{1}-i a \epsilon / 2, z_{2}-i b \epsilon / 2\right)=1-\delta_{a b}\left(z_{1}-z_{2}\right)
$$

with $\delta_{a b}(z)$ the contribution of the singularities pinching the integration contour, which becomes a delta-like function as $g \rightarrow \infty$,

$$
\int \frac{d z}{2 \pi \epsilon} \delta_{a b}(z)=\frac{a b}{a+b} .
$$

\footnotetext{
${ }^{12}$ This is the contour pinching which is alluded to in [10].
} 
Evaluating the contribution from $\delta_{a, b}(z)$ will be equivalent to the clustering procedure.

Let us analyse some simple examples in order to illustrate the idea. Let us organise the sum of (5.2) as

$$
\left[\mathcal{C}^{\bullet \bullet 0}\right]^{\text {bottom }}=\sum_{n=1}^{\infty} \mathrm{B}_{n}, \quad \mathrm{~B}_{n}=\sum_{\vec{n}: \sum_{a} n_{a} a=n} \frac{\mathrm{B}_{s c}[\vec{n}]}{\prod_{a} n_{a} !}
$$

and consider $\mathrm{B}_{1}, \mathrm{~B}_{2}$ and $\mathrm{B}_{3}$. We have

$$
\begin{aligned}
\mathrm{B}_{1} & =\int \frac{d z}{2 \pi \epsilon} \mathrm{T}_{1}(z)=\int \frac{d z}{2 \pi \epsilon} \mathrm{t}_{1}(z) \\
\mathrm{B}_{2} & =\frac{1}{2} \int \frac{d z_{1} d z_{2}}{(2 \pi \epsilon)^{2}} \mathrm{~T}_{1}\left(z_{1}\right) \mathrm{T}_{1}\left(z_{2}\right) \Delta_{11}\left(z_{1}, z_{2}\right)+\frac{1}{2} \int \frac{d z}{2 \pi \epsilon} \mathrm{T}_{2}(z) .
\end{aligned}
$$

Using (5.33) the first term of $\mathrm{B}_{2}$ can be re-written as

$$
\frac{1}{2} \int \frac{d z_{1} d z_{2}}{(2 \pi \epsilon)^{2}} \mathrm{~T}_{1}\left(z_{1}\right) \mathrm{T}_{1}\left(z_{2}\right)-\frac{1}{4} \int \frac{d z_{1}}{2 \pi \epsilon} \mathrm{T}_{1}\left(z_{1}\right)^{2}
$$

where in the first term of (5.36), the integrals for $z_{1}$ and $z_{2}$ are independent. The second term of (5.36) comes from taking the pole or clustering. Using the second line in $(5.28), \mathrm{B}_{2}$ can be written as

$$
\mathrm{B}_{2}=\frac{1}{2}\left(\int \frac{d z}{2 \pi \epsilon} \mathrm{t}_{1}(z)\right)^{2}+\frac{1}{4} \int \frac{d z}{2 \pi \epsilon} \mathrm{t}_{2}(z) .
$$

In appendix $\mathrm{E}$ we show that the third term in the expansion is equal to

$$
\mathrm{B}_{3}=\frac{1}{3 !}\left(\int \frac{d z}{2 \pi \epsilon} \mathrm{t}_{1}(z)\right)^{3}+\frac{1}{4}\left(\int \frac{d z}{2 \pi \epsilon} \mathrm{t}_{1}(z)\right)\left(\int \frac{d z}{2 \pi \epsilon} \mathrm{t}_{2}(z)\right)+\frac{1}{9} \int \frac{d z}{2 \pi \epsilon} \mathrm{t}_{3}(z) .
$$

These three terms are consistent with the expansion of the exponential of a sum of dilogarithms. In the next section we prove that the full expression is indeed the exponential of a sum of dilogarithms.

Combinatorics of clusters of bound states. Now we can perform the clustering of bound states in the mirror channel in full generality following the pattern we have just explained. We start with the sum over bound states with the interactions $\Delta_{a b}$. After taking into account the contribution of the poles $\delta_{a b}$, which is equivalent to clustering, we re-organise the bound states. The final result is again a sum over bound states, but without the interactions $\delta_{a b}$ and with a different combinatorics factor. It is this combinatorics factor that we are going to determine next. 
As a first step, we consider the problem of clustering $k$ bound states into a single bound state. The initial bound state configuration can be labeled by $\vec{n}=\left\{n_{1}, n_{2}, \cdots\right\}$ where $n_{a}$ is the number of the bound states of length $a$. Let us denote

$$
n=\sum_{a} n_{a} a, \quad k=\sum_{a} n_{a}
$$

so that $k$ is the initial number of bound states and $n$ is the length of the resulting bound state. The big bound state is obtained by clustering the following product

$$
\prod_{a} \prod_{j=1}^{n_{a}} \frac{\mathrm{T}_{a}\left(z_{j}^{a}\right)}{a}
$$

The clustering rule for the bound states of length $a$ and $b$ is given, according to (5.33), by

$$
\frac{\mathrm{T}_{a}\left(z_{j}\right)}{a} \times \frac{\mathrm{T}_{b}\left(z_{k}\right)}{b} \rightarrow-\frac{\mathrm{T}_{a}\left(z_{j}\right) \mathrm{T}_{b}\left(z_{j}\right)}{a+b} .
$$

Therefore, the clustering of $k$ bound states with the initial configuration $\vec{n}$ gives

$$
\prod_{a} \prod_{j=1}^{n_{a}} \frac{\mathrm{T}_{a}\left(z_{j}^{a}\right)}{a} \rightarrow(-1)^{k-1} \frac{(k-1) !}{n} \prod_{a} \mathrm{~T}_{a}\left(z_{1}\right)^{n_{a}}
$$

where the factorial $(k-1)$ ! takes into account different orders of clustering. The last expression, when summed over all the configurations of initial bound states with weight $n$, gives, upon using (5.30),

$$
\sum_{\vec{n}: \sum_{a} n_{a} a=n}(-1)^{k-1} \frac{(k-1) !}{n} \prod_{a} \frac{\mathrm{T}_{a}(z)^{n_{a}}}{n_{a} !}=\frac{\mathrm{t}_{n}}{n^{2}} .
$$

We recognise here the factor $1 / n^{2}$ which is necessary to reconstruct the sought-off dilogarithm, and which appears as a non-trivial combination of the $1 / n$ factor from the measure of integration $\mu_{n}(z)$ and the $1 / n$ factor in the $\mathrm{t}-\mathrm{T}$ relations (5.30).

In order to complete the proof, we have to also consider the generic case when the set of bound states $\vec{n}=\left\{n_{1}, n_{2}, \cdots\right\}$ cluster into bound states $\vec{d}=\left\{d_{1}, d_{2}, \cdots\right\}$ where $d_{l}$ is the number of bound state of length $l$. Again, as in (3.33), we find it helpful to use alternatively the non-decreasing sequence $\left\{q_{1}, q_{2}, \cdots, q_{m}\right\}$ to caracterise $\vec{d}$. The total number of cluster in the final state is denoted by $m=\sum_{l} d_{l}$. Each bound state $q_{j}$ in the final set is obtained from fusing a subset $\vec{n}^{(j)}=\left\{n_{1}^{(j)}, n_{2}^{(j)}, \cdots\right\}$ of the initial bound states $\vec{n}=\left\{n_{1}, n_{2}, \cdots\right\}$. To prepare the clustering we split the initial factor as

$$
\mathrm{T}_{1}^{n_{1}} \mathrm{~T}_{2}^{n_{2}} \mathrm{~T}_{3}^{n_{3}} \ldots=\prod_{j=1}^{m}\left(\mathrm{~T}_{1}^{n_{1}^{(j)}} \mathrm{T}_{2}^{n_{2}^{(j)}} \mathrm{T}_{3}^{n_{3}^{(j)}} \ldots\right)
$$


such that

$$
\sum_{a} n_{a}^{(j)} a=q_{j}, \quad \sum_{a} n_{a}^{(j)}=k_{j}, \quad \sum_{j=1}^{m} n_{a}^{(j)}=n_{a}
$$

Let us now count the symmetry factors. First, there is the factor from the definition (5.2). Second, a permutation of the bound states of the same length in $\left\{q_{1}, q_{2}, \cdots, q_{m}\right\}$ leads to the same representation $\vec{d}$ and we have to take care of this redundancy as well. Together, these symmetry factors are given by

$$
\prod_{a} \frac{1}{n_{a} !} \prod_{l} \frac{1}{d_{l} !}
$$

Next, there is a factor

$$
\frac{n_{a} !}{\prod_{j=1}^{k} n_{a}^{(j) !}}=\left(\begin{array}{c}
n_{a} \\
n_{a}^{(1)}
\end{array}\right)\left(\begin{array}{c}
n_{a}-n_{a}^{(1)} \\
n_{a}^{(2)}
\end{array}\right) \ldots
$$

coming from distributing $n_{a}$ bound states of type $a$ into the different sets $n_{a}^{(j)}$. By clustering $\vec{n}^{(j)}$ into a single bound state of length $q_{j}$ one gets the same factor as in (5.43) for each $j$. We have then

$$
\begin{gathered}
\mathrm{B}_{\vec{d}}=\prod_{l} \frac{1}{d_{l} !} \times \prod_{j=1}^{m} \int \frac{d z_{j}}{2 \pi \epsilon} \sum_{\vec{n}^{(j)}: \sum_{a} n_{a}^{(j)} a=q_{j}}(-1)^{k_{j}-1} \frac{\left(k_{j}-1\right) !}{q_{j}} \prod_{a} \frac{\mathrm{T}_{a}^{n_{a}^{(j)}}\left(z_{j}\right)}{n_{a}^{(j)} !} \\
=\prod_{l} \frac{1}{d_{l} !} \times \prod_{j=1}^{m} \int \frac{d z_{j}}{2 \pi \epsilon} \frac{\mathrm{t}_{q_{j}}\left(z_{j}\right)}{q_{j}^{2}}
\end{gathered}
$$

where we have used (5.30) repeatedly. $\mathrm{B}_{\vec{d}}$ is the contribution of the bound states after clustering $\vec{d}$, not to be confused with the contribution before clustering, $\mathrm{B}[\vec{n}]$. Using the same argument as in section 3.4 we can write the final answer as

$$
\left[\mathcal{C}^{\bullet \circ}\right]^{\text {bottom }}=\sum_{\vec{d}} \mathrm{~B}_{\vec{d}}=\exp \int \frac{d z}{2 \pi \epsilon} \sum_{n} \frac{\mathrm{t}_{n}(z)}{n^{2}}
$$

\subsection{The $\mathfrak{s u}(2)$ bottom mirror contribution}

The result after clustering is remarkably similar to the result for the asymptotic case (3.36), with the exception that we are now in the zero shift limit and that $\mathrm{t}_{n}(z)$ is composed from four terms. In the $\mathfrak{s u}(2)$ sector $^{13}$

$$
\mathrm{t}_{n}^{\mathfrak{s u}(2)}=\tilde{g}^{n}\left(1+1-f^{n}-\bar{f}^{n}\right) .
$$

\footnotetext{
${ }^{13}$ The combination of these four terms for $n=1$ in the $\mathfrak{s l}(2)$ sector was considered in appendix $\mathrm{M}$ of [1].
} 
Here we obtain the full expansion of the dilogarithm

$$
\begin{aligned}
\log \left[\mathcal{C}^{\bullet \circ 0}\right]^{\text {bottom }} & =\int_{-1}^{1} \frac{d z}{2 \pi \epsilon}\left(\operatorname{Li}_{2}\left[e^{i\left(\hat{p}^{(2)}(1 / x)+\hat{p}^{(3)}(1 / x)-\hat{p}^{(1)}(1 / x)\right)}\right]-\operatorname{Li}_{2}\left[e^{i\left(\tilde{p}^{(2)}(1 / x)+\tilde{p}^{(3)}(1 / x)-\tilde{p}^{(1)}(1 / x)\right)}\right]\right) \\
& -\int_{-1}^{1} \frac{d z}{2 \pi \epsilon}\left(\operatorname{Li}_{2}\left[e^{i\left(\hat{p}^{(2)}(x)+\hat{p}^{(3)}(x)-\hat{p}^{(1)}(x)\right)}\right]-\operatorname{Li}_{2}\left[e^{i\left(\tilde{p}^{(2)}(x)+\tilde{p}^{(3)}(x)-\tilde{p}^{(1)}(x)\right)}\right]\right)
\end{aligned}
$$

where $\hat{p}_{i}(z), \tilde{p}_{i}(z)$ with $i=1,2,3$ are the AdS and the sphere parts of the quasi-momenta of the three operators respectively. Contrary to [1], here it is the sphere part of the quasimomenta which is non-trivial,

$$
\begin{aligned}
& \tilde{p}^{(1)}(x)=\frac{1}{2} \Delta p(x)-\mathcal{G}_{\mathbf{u}}(x), \quad \tilde{p}^{(k)}(x)=\frac{1}{2} L_{k} p(x), \quad k=2,3, \\
& \hat{p}^{(1)}(x)=\frac{1}{2} \Delta p(x), \quad \hat{p}^{(k)}(x)=\frac{1}{2} L_{k} p(x), \quad k=2,3,
\end{aligned}
$$

where $p(x)=x / g\left(x^{2}-1\right)$. In writing (5.51) we also have used $p(1 / x)=-p(x)$, as well as the functional equation of the dilogarithm. The integration path in (5.51) is understood as follows: choosing the determination $1 / x(z)$ for the Zhukovsky variable in the first line means that we integrate below the cut, on the contour $U_{-}$, while choosing the determination $x(z)$ means we are integrating on the contour $U_{+}$above the cut. The integral (5.51) can be recast as a contour integral on the contour $U$ surrounding the cut counterclockwise,

$\log \left[\mathcal{C}^{\bullet \circ \circ}\right]^{\text {bottom }}=\oint_{U} \frac{d z}{2 \pi \epsilon}\left(\operatorname{Li}_{2}\left[e^{i\left(\hat{p}^{(2)}(x)+\hat{p}^{(3)}(x)-\hat{p}^{(1)}(x)\right)}\right]-\operatorname{Li}_{2}\left[e^{i\left(\tilde{p}^{(2)}(x)+\tilde{p}^{(3)}(x)-\tilde{p}^{(1)}(x)\right)}\right]\right)$.

This result agrees with the second line of the strong coupling string result (2.13) obtained in [21].

\subsection{The $\mathfrak{s l}(2)$ bottom mirror contribution}

The computation of the wrapping corrections in the $\mathfrak{s l}(2)$ sector goes along the lines of the computation in the $\mathfrak{s u}(2)$ sector, the only difference being that the hexagon amplitude $h(u, v)$ and transfer matrix get replaced with their $\mathfrak{s l}(2)$ counterparts. The measure $\mu(u)$ and the factor $H_{a, b}(u, v)$ are exactly the same as in the $\mathfrak{s u}(2)$ case, while $h_{\mathfrak{s l}(2)}(u, v)$ is defined in $(3.50)$, so that

$$
g_{a}^{\mathfrak{s l}(2)}(u) \equiv \frac{e^{i p_{a}(u) \ell_{B}}}{h_{a 1}^{\mathfrak{s l}(2)}(u, \mathbf{u})}=\frac{R^{(-)[-a]}}{R^{(-)[+a]}} \tilde{g}_{a}^{\mathfrak{s u}(2)}(u)
$$


We deduce that the analyticity properties of $g_{a}^{\mathfrak{s l}(2)}(u)$ are the same as the ones for $\tilde{g}_{a}^{\mathfrak{s u}(2)}(u)$. The transfer matrices $T_{a}^{\mathfrak{s l}(2)}(u)$ are defined in terms of the generating functional

$$
\sum_{a=0}^{\infty}(-1)^{a} T_{a}^{[a-1]}(u) \mathrm{D}^{2 a}=\left(1-Y_{0,1} \mathrm{D}^{2}\right)^{-1}\left(1-X_{1,1} \mathrm{D}^{2}\right)\left(1-X_{2,1} \mathrm{D}^{2}\right)\left(1-Y_{2,2} \mathrm{D}^{2}\right)^{-1}
$$

$$
\text { with } \quad X_{2,1}=X_{1,1}=\frac{R^{(+)-}}{R^{(-)-}}=f^{-}, \quad Y_{2,2}=1, \quad Y_{0,1}=\frac{B^{(+)+}}{B^{(-)+}} \frac{R^{(+)-}}{R^{(-)}-}=\frac{f^{-}}{\bar{f}^{+}}, \quad \mathrm{D}^{2}=e^{i \epsilon \partial_{u}} .
$$

The explicit expression of the $\mathfrak{s l}(2)$ transfer matrices, correctly normalised, is more complicated than (5.23) and it is given by equation (H1) in [1]. Even if it's not immediately obvious, the matrices $T_{a}(u)$ have only two cuts situated at distance $i a \epsilon$. The remaining cuts vanish by virtue of the symmetry of $T_{a}(u)$ with respect to the exchange $x^{[k]} \leftrightarrow 1 / x^{[k]}$ for $k \neq \pm a$. Again, we can redefine the quantities

$$
\mathrm{T}_{a}^{\mathfrak{s l}(2)}(u) \equiv\left[g_{a}(u) T_{a}(u)\right]^{\mathfrak{s l}(2)}
$$

which have the required analytical properties to allow continuation to the mirror dynamics. In the strong coupling limit, the matrices $\mathrm{T}_{a}(u)$ defined as above obey again the $\mathrm{t}-\mathrm{T}$ relations (5.28), this time with

$$
\mathrm{t}_{n}^{\mathfrak{s l}(2)}=(-1)^{n+1}(g / f)^{n}\left(2-f^{n}-\bar{f}^{n}\right) .
$$

The sign $(-1)^{n}$ compensates the one in (5.3), while the extra minus sign accounts for the exchange of the sector carrying the non trivial excitations. All in all, the result of summing out the mirror excitation gives an expression identical to (5.51) and (5.53), with the quasimomenta being now

$$
\begin{gathered}
\hat{p}^{(1)}(x)=\frac{1}{2} \Delta p(x)+\mathcal{G}_{\mathbf{u}}(x), \quad \hat{p}^{(k)}(x)=\frac{1}{2} L_{k} p(x), \quad k=2,3 \\
\tilde{p}^{(k)}(x)=\frac{1}{2} L_{k} p(x), \quad k=1,2,3,
\end{gathered}
$$

Again, the result agrees with the second line of the strong coupling string result (2.15) obtained in [21].

\section{$6 \quad$ Fredholm determinants and free fermions}

As it was already remarked in [1], at strong coupling the bi-local scalar factors become the same as those at tree level. This allows to recycle the techniques developed for the tree-level structure constant in $[6-9,48]$. The techniques in question, which we will refer 
to as determinant methods, reformulate the problem in terms of several equivalent objects, Fredholm determinants, free fermions and chiral Toda theory, which are related to the fermionic system by bosonization.

Although we already obtained the solution of our problem by the combinatorics of clusters, it is potentially useful to give an alternative derivation, which will recast the solution in a nice operator form and potentially give us intuition about how to adjust our method to more general class of correlation functions. To our surprise, the determinant methods work with remarkable efficiency for the resummation of the mirror channel in section 5.2, where they led naturally to an object which could be named "quantum spectral determinant". The quantum spectral determinant is the determinant in the functional space of a finite-difference operator, obtained by replacing the spectral parameter in the spectral (super)determinant with the shift operator $\mathrm{D}^{2}$. The finite-difference operator in question already appeared in the literature [43-45] in the guise of exact generating function for the transfer matrices, $c f . \quad$ (5.22) and (5.54). Our analysis shows that this operator is not just a formal expansion, but has a deeper meaning and will certainly play important role in building analytic methods to study the correlation functions.

It is worth mentioning that the fermionic formalism allows to reveal a hidden integrable structure of the structure constant and identify it (at strong coupling) as a $\tau$-function of the KP integrable hierarchy ${ }^{14}$. This means that the structure constant satisfies an infinite series of non-linear PDE as a function of the conserved quantities characterizing the operators $\mathcal{O}_{1}, \mathcal{O}_{2}$ and $\mathcal{O}_{3}$. This integrable structure is not really necessary in the context of this work, because we have already found the explicit solution, but it could be useful for studying more complicated objects as the I-I-I type structure constant.

In this section we will focus on the description via Fredholm determinants. We will show that not only the leading term in the semiclassical limit, but the whole semiclassical series can be given a condensed formulation in terms of a Fredholm determinant. We will only briefly mention the fermionic and the bosonic QFT realizations of the structure constant. In particular, we will give an interpretation of the semiclassical result (3.40) as the grand canonical partition function of free fermions living on a line. More details about the representation in terms of chiral fermions can be found in $[9,50]$. Our goal in this section is mainly to give an intuitive explanation of the combinatorial factors obtained in sections 3 and 5.

\footnotetext{
${ }^{14}$ Such a statement has been made in [49]; we evoke a different realization of the $\tau$-function.
} 


\subsection{Multiple contour integrals as a Fredholm determinant}

With the help of the Cauchy identity

$$
\frac{1}{\epsilon^{n}} \prod_{j<k}^{n} \frac{\left(z_{j}-z_{k}\right)^{2}}{\left(z_{j}-z_{k}\right)^{2}+\epsilon^{2}}=\operatorname{det}_{j, k}\left(\frac{i}{z_{j}-z_{k}+i \epsilon}\right)
$$

the $n$-th term in the expansion (3.16) can be put in the form

$$
I_{n}=\frac{(-1)^{n}}{n !} \int_{\mathcal{C}} \prod_{j=1}^{n} \frac{d z_{j}}{2 \pi i} \operatorname{det}\left(\frac{F\left(z_{j}\right)}{z_{j}-z_{k}+i \epsilon}\right) .
$$

Then the sum $\mathscr{A}=1+I_{1}+I_{2}+\ldots$ in (3.16) takes the form of the expansion of a Fredholm determinant,

$$
\mathscr{A}=\operatorname{Det}(1-K) \equiv \sum_{n=0}^{\infty} \frac{(-1)^{n}}{n !} \oint_{\mathcal{C} \times n} d z_{1} \ldots d z_{n} \operatorname{det}_{j k} K\left(z_{j}, z_{k}\right)
$$

with the integral operator $K$ defined as ${ }^{15}$

$$
K \psi(u)=\oint_{\mathcal{C}} d z K(u, z) \psi(z), \quad K(u, v)=\frac{1}{2 \pi i} \frac{F(u)}{u-v+i \epsilon} .
$$

The logarithm of the Fredholm determinant is a series of multiple integrals

$$
\begin{aligned}
\log \operatorname{Det}(1-K) & =-\oint_{\mathcal{C}} d z K(z, z)-\frac{1}{2} \oint_{\mathcal{C}^{\times 2}} d z_{1} d z_{2} K\left(z_{1}, z_{2}\right) K\left(z_{2}, z_{1}\right) \\
& -\frac{1}{3} \oint_{\mathcal{C}^{\times 3}} d z_{1} d z_{2} d z_{3} K\left(z_{1}, z_{2}\right) K\left(z_{2}, z_{3}\right) K\left(z_{3}, z_{1}\right)+\ldots
\end{aligned}
$$

The Fredholm operator in (6.4) can be represented in the factorised form

$$
K=F \mathrm{D}^{2} \mathrm{P}_{+}
$$

where $\mathrm{D}^{2}=e^{i \epsilon \partial}$ is the shift operator and $\mathrm{P}_{+}$is the Cauchy transform

$$
\mathrm{P}_{+} \psi(u)=\oint_{\mathcal{C}} d z \frac{1}{2 \pi i} \frac{1}{u-z+i 0} \psi(z) .
$$

The semiclassical limit of (6.5) has been studied in [9], where the first two terms of the semiclassical expansion

$$
\log \mathscr{A}=\frac{1}{\epsilon} \mathscr{F}_{0}+\mathscr{F}_{1}+\epsilon \mathscr{F}_{2}+\ldots
$$

have been obtained. Compared to the analysis in [9], the method of clustering described in section 3.3 is more powerful because it allows to obtain, after some combinatorics, the whole semiclassical expansion.

\footnotetext{
${ }^{15}$ In the particular case when $F$ is a constant in the interval $[-L / 2, L / 2]$ and vanishes outside this interval, the Fredholm determinant has been computed for by Michel Gaudin [51].
} 


\subsection{The leading term by Fredholm determinant}

The operator formalism provides an alternative (shorter, but not rigorous) derivation of the leading term of the semiclassical expansion. It is based on the following approximation for the $n$-th power of the operator $K$,

$$
K^{n} \equiv\left(F \mathrm{D}^{2} \mathrm{P}_{+}\right)^{n}=\left(F \mathrm{D}^{2}\right)^{n} \mathrm{P}_{+}+\text {subleading. }
$$

Replacing $\mathrm{P}_{+}$with the identity ${ }^{16}$ on the rhs is equivalent to retaining only the contribution of the poles at $z_{j+1}=z_{j}+i \epsilon$. The operator $K^{n}$ in this approximation is an integral operator with kernel

$$
K^{n}\left(z_{1}, z_{2}\right)=\frac{1}{2 \pi i} \frac{F_{n}\left(z_{1}\right)}{z_{1}-z_{2}+i n \epsilon},
$$

where the function $F_{n}(u)$ is defined in (3.25). Now we can evaluate the sum in (6.5) as

$$
\operatorname{Tr} \log (1-K)=-\sum_{n=1}^{\infty} \int d z K^{n}(z, z)=\frac{1}{\epsilon} \oint_{\mathcal{C}} \frac{d z}{2 \pi} \sum_{n \geq 1} \frac{F_{n}(u)}{n^{2}},
$$

which coincides with the expression (3.38) obtained by the clustering method. This operator representation will be particularly useful when computing the wrapping corrections.

\subsection{The leading term as the free energy of a Fermi gas}

The above result can be given an intuitive explanation by the analogy with a grand ensemble of one-dimensional fermions living on the contour $\mathcal{C}$. We place for simplicity the integration contour $\mathcal{C}$ along the real axis, from left to right, and close it at infinity in the upper half-plane. This simplifying assumption does not change the short distance behavior, in particular the mechanism for clustering. The Cauchy transform (6.7) acts as the projection operator to the functions analytic in the upper half-plane, which we choose as our Hilbert space. Such are the wave functions of the right-moving fermions. The Hilbert space $\mathcal{H}_{+}$of the right-movinf fermions is spanned by the plane waves

$$
\psi_{k}(z) \equiv\langle z \mid k\rangle=e^{i k z / \epsilon} \quad \text { with } k>0,
$$

while the dual Hilbert space $\mathcal{H}_{-}$of the left-moving fermions is spanned by the plane waves

$$
\bar{\psi}_{k}(z) \equiv\langle k \mid z\rangle=e^{-i k z / \epsilon} \quad \text { with } k>0 .
$$

\footnotetext{
${ }^{16}$ Imagine that the contour $\mathcal{C}$ is deformed so that the new contour obtained from $\mathcal{C}$ by translation $z \rightarrow z+i \epsilon$ is inside $\mathcal{C}$. For that the contour should be stretched to $+i \infty$. Then the operator $\mathrm{P}_{+}$is the identity operator in the space $\mathcal{H}_{+}$of the functions analytic inside $\mathcal{C}$.
} 
The plane wave decomposition of the identity operator in $\mathcal{H}_{+}$is

$$
\int_{0}^{\infty}|k\rangle\langle k|=\mathrm{P}_{+}, \quad\left\langle z_{1}\left|\mathrm{P}_{+}\right| z_{2}\right\rangle \equiv \int_{0}^{\infty} \frac{d k}{2 \pi \epsilon}\left\langle z_{1} \mid k\right\rangle\left\langle k \mid z_{2}\right\rangle=\frac{1}{2 \pi i} \frac{1}{z_{1}-z_{2}+i 0} .
$$

The fermions are allowed to have only positive energy $k$ and their wave functions are the plane waves (6.12). The grand canonical partition function of the fermionic ensemble is given by the Fredholm determinant (6.3), with the Fredholm kernel (6.4) playing the role of a density matrix in coordinate representation:

$$
K(z, u)=\int_{0}^{\infty} \frac{d k}{2 \pi \epsilon} F(z)\langle z \mid k\rangle e^{-k}\langle k \mid u\rangle .
$$

The coordinate $u$ and the momentum $\hat{k}=-i \epsilon \partial / \partial u$ are canonically canonically conjugate operators

$$
[u, \hat{k}]=i \epsilon
$$

and the limit $\epsilon \rightarrow 0$ corresponds precisely to the semiclassical limit of this free fermion system. In the semiclassical limit the logarithm of the function $F$ can be considered as an external potential which adds to the kinetic energy $k$ of the fermion:

$$
E_{\mathrm{cl}}(u, k)=k-\log F(u) .
$$

As is well-known, the grand potential in the semiclassical limit is given by the integral over the phase space of the right-moving fermions,

$$
\ln \mathscr{A} \simeq-\frac{1}{\epsilon} \oint_{\mathcal{C}} \frac{d u}{2 \pi} \int_{0}^{\infty} d k \ln \left(1-e^{-E_{\mathrm{cl}}(u, k)}\right) .
$$

Performing the integration over $k$, we reproduce the leading term in (3.39). Note that the discussion here is in complete parallel with the Fermi gas approach to the ABJM matrix model [19]. In that context, the subleading corrections can be determined by the WignerKirkwood expansion $[52,53]$, which can be carried out e.g. by the method of co-adjoint orbits [54]. It would be an interesting future problem to apply it to the three-point function and try to compute the subleading corrections systematically ${ }^{17}$.

\subsection{Semiclassical expansion by nested Fredholm determinant}

It happens that the exact result (3.36) obtained by the nesting method can be expressed in terms of a (different) Fredholm determinant. Applying the Cauchy identity as in (6.1), we

\footnotetext{
${ }^{17}$ Conversely, we can apply the clustering method to the AB JM partition function and obtain the leading term in the M-theory regime. This is demonstrated in Appendix F.
} 
can write the integrand/summand in the $n$-th term of the series (3.36) as

$$
\prod_{j=1}^{n} \frac{F_{q_{j}}\left(z_{j}\right)}{\epsilon q_{j}^{2}} \prod_{j<l}^{n} \Delta_{q_{j}, q_{l}}\left(z_{j}, z_{l}\right)=(-1)^{n} \operatorname{det}_{j, l} K_{q_{j}, q_{l}}\left(z_{j}, z_{l}\right),
$$

where the matrix kernel $\hat{K}=\left\{K_{q_{1}, q_{2}}\left(z_{1}, z_{2}\right)\right\}_{q_{1}, q_{2} \geq 1}$ is defined by

$$
K_{q_{1}, q_{2}}\left(z_{1}, z_{2}\right)=\frac{1}{2 \pi i} \frac{F_{q_{1}}\left(z_{1}\right)}{q_{1}} \frac{1}{z_{1}-z_{2}+i \epsilon q_{1}} .
$$

This turns (3.36) into the expansion of a generalised Fredholm determinant $\operatorname{Det}(1-\hat{K})$. In addition, this is a "nested Fredholm determinant" in the sense that the integration for the $n$-th term of the expansion is performed for a nested configuration of contours $\mathcal{C}_{1} \lessdot \mathcal{C}_{2} \lessdot \ldots \lessdot \mathcal{C}_{n}$ associated with the contour $\mathcal{C}$. The relation $\mathcal{C}_{1} \lessdot \mathcal{C}_{2}$ means that $\mathcal{C}_{1}$ is inside $\mathcal{C}_{2}{ }^{18}$ All contours $\mathcal{C}_{k}$ from the nested configuration are obtained from the contour $\mathcal{C}$ by a continuous deformation without crossing poles or other singularities of the function $F(z)$.

It is important that the matrix elements of the generalised Fredholm kernel (6.20) depend only on the first index $q_{1}$. This allows to replace the matrix kernel $\hat{K}$ with a scalar kernel, but of different functional form. Indeed, in the expansion

$$
\begin{aligned}
\log \operatorname{Det}(I-\hat{K})_{\text {nested }}= & -\sum_{q \geq 1} \oint_{\mathcal{C}} d z K_{q, q}(z, z) \\
& -\frac{1}{2} \sum_{q_{1}, q_{2} \geq 1} \oint_{\mathcal{C}_{1}<\mathcal{C}_{2}} d z_{1} d z_{2} K_{q_{1}, q_{2}}\left(z_{1}, z_{2}\right) K_{q_{2}, q_{1}}\left(z_{2}, z_{1}\right)-\ldots
\end{aligned}
$$

the sums over $q_{i}$ decouple and the matrix kernel $\hat{K}$ can be replaced with a scalar kernel

$$
\mathbb{K}\left(z_{1}, z_{2}\right)=\frac{1}{2 \pi i} \sum_{q=1}^{\infty} \frac{F_{q}\left(z_{1}\right)}{q} \frac{1}{z_{1}-z_{2}+i q \epsilon} .
$$

As a consequence, the quantity $\mathscr{A}$ is given by a nested Fredholm determinant of the scalar kernel $\mathbb{K}$ :

$$
\mathscr{A}=\operatorname{Det}(\mathrm{I}-\mathbb{K})_{\text {nested }}
$$

A convenient operator expression for $\mathscr{A}$ is obtained by representing the kernel $\mathbb{K}$ in a factorised form, as we did for $K$ in (6.6),

$$
\mathbb{K}=\sum_{q=1}^{\infty} \frac{F_{q}}{q} \mathrm{D}^{2 q} \mathrm{P}_{+}=\sum_{q=1}^{\infty} \frac{\left(F \mathrm{D}^{2}\right)^{q}}{q} \mathrm{P}_{+}=-\log \left(1-F \mathrm{D}^{2}\right) \cdot \mathrm{P}_{+}
$$

\footnotetext{
${ }^{18}$ This also means that the two contours remain separated at finite distance in the limit $\epsilon \rightarrow 0$.
} 


\subsection{CFT representation}

The representation as a determinant enables us to use the formalism of quantum field theory and 2D conformal field theory as in the case of random matrix models [55]. To formulate the semiclassical expansion in QFT terms, we first identify the fermionic system associated with the determinant (6.23) and transform it into a bosonic collective field theory. Let us first remind the representation, given in [56], of the original "unnested" Fredholm determinant (6.3) as an expectation value of two-dimensional chiral fermion $\psi, \bar{\psi}$ with with two-point function $\langle 0|\bar{\psi}(z) \psi(u)| 0\rangle=1 /(z-u)$ and interacting with a common external potential determined by the function $F$. We have (for the details we refer to $[9,56]$ )

$$
\mathscr{A}=\left\langle 0\left|\exp \oint_{\mathcal{C}} \frac{d z}{2 \pi i} F(z) \bar{\psi}(z) \mathrm{D}^{2} \psi(z)\right| 0\right\rangle .
$$

The nested Fredholm determinant (6.23) has the Fock space representation

$$
\mathscr{A}=\left\langle 0\left|\left(\exp \int_{\mathcal{C}} \frac{d z}{2 \pi i} \bar{\psi}(z) \log \left(1-F(z) \mathrm{D}^{2}\right) \psi(z)\right)_{\text {nested }}\right| 0\right\rangle,
$$

where the "nested exponent" is defined as

$$
\left(\exp \int_{\mathcal{C}} d z f(z)\right)_{\text {nested }}=\sum_{k \geq 0} \frac{1}{k !} \oint_{\mathcal{C}_{1}<\ldots<\mathcal{C}_{k}} d z_{1} \ldots d z_{k} f\left(z_{1}\right) \ldots f\left(z_{k}\right) .
$$

This simple fermionic system can be transformed into a chiral Toda-like theory by twodimensional bosonization, $\psi \rightarrow e^{\phi}, \bar{\psi} \rightarrow e^{-\phi}$. In the bosonic theory, the $q$-clusters are represented by the vertex operators

$$
V_{q}(z)=e^{-\phi(z)} e^{\phi(z+i q \epsilon)}
$$

which are electrically neutral but have dipole charge $q \epsilon$. The function $\Delta_{m n}(u, v)$ defined by (3.19) is the correlation function of the dipoles $V_{m}(u)$ and $V_{n}(v)$. The leading term is the free energy of the gas of dipoles in the external potential $-\log F$, in the dilute gas approximation. Taking into account the interaction generates a series of connected graphs which is the QFT formulation of the cumulant, or Mayer, expansion. For instance, the subdominant term in (3.39) is given by the connected correlation function of two vertex operators.

\subsection{Wrapping corrections by Fredholm determinant}

The sum over the mirror particles has a form very similar to the asymptotic contribution after being reformulated in terms of multiple contour integrals. It is possible that this latter 
formulation is more fundamental than the sum over partitions. Remarkably, the strong coupling limit of the contribution of the mirror particles associated with the bottom edge, eq. (5.2), can also be converted to a Fredholm determinant. The derivation is again based on converting the bi-local products into determinants with the help of Cauchy identity. All statements and derivations in this subsection are valid only to the leading order in the semiclassical strong coupling limit. It is of course desirable to develop these techniques beyoud the leading order, but this is far beyond the scope of the research presented in this paper. Since we are interested only in the leading behavior, we can rescale $\epsilon$ so that $2 g \epsilon=1$ and then take the limit $\epsilon \rightarrow 0$.

Let us consider for definiteness the case $\mathfrak{s u}(2)$. The analytical properties of the Tmatrices allow to perform a shift $T_{a} \rightarrow T_{a}^{[-a]}$ in the original integral along the real axis in the mirror dynamics. This leads to (5.31) with $z^{a} \rightarrow z^{a}+i a \epsilon / 2$. After the shift, the bi-local factors $\Delta_{a b}$, which give the asymptotics of $H_{a, b}^{\gamma}$, transform to

$$
\Delta_{a b}(z-i a \epsilon / 2, z-i b \epsilon / 2) \rightarrow \Delta_{a b}\left(z-i a \epsilon, z^{\prime}-i b \epsilon\right)=\Delta_{b a}\left(z, z^{\prime}\right)
$$

and (5.31) can be written equivalently as ${ }^{19}$

$$
\mathrm{B}_{s c}[\vec{n}] \rightarrow \int_{U_{-}} \prod_{a} \prod_{j=1}^{n_{a}} \frac{d z_{j}^{a}}{2 \pi a \epsilon} \mathrm{T}_{a}^{[-a]}\left(z_{j}^{a}\right) \prod_{\substack{a \\ 1 \leq i<j \leq n_{a}}} \Delta_{a, a}\left(z_{i}^{a}, z_{j}^{a}\right) \times \prod_{\substack{a<b \\ 1 \leq i \leq n_{a} \\ 1 \leq j \leq n_{b}}} \Delta_{b, a}\left(z_{i}^{a}, z_{j}^{b}\right)
$$

The next step is to write the re-organise the sum of all $B[\vec{n}]$ in $(5.2)$ as a sum over all possible sets $I$ of double indices $\{i, a\}$, with $a$ and $i$ being otherwise unrestricted positive integers. Then for any set $I$ we express the product of the factors $\Delta$ in the rhs of (5.31) as a Cauchy determinant of size $k=|I|$. This allows to write the sum over all $B[\vec{n}]$ as

$$
\left[\mathcal{C}_{123}^{\bullet \circ 0}\right]_{\mathfrak{s u}(2)}^{\text {bottom }} \rightarrow \sum_{k=0}^{\infty} \frac{1}{k !} \sum_{|I|=k} \int_{U_{-}} \prod_{\{i, a\} \in I} \frac{d z_{j}^{a}}{2 \pi i} \operatorname{det}_{k \times k} \frac{\mathrm{T}_{a}^{[-a]}\left(z_{j}^{a}\right)}{z_{i}^{a}-z_{j}^{b}-i a \epsilon}
$$

Reasoning in the same way as in the derivation of (6.23), we express the right-hand side as a Fredholm determinant

$$
\operatorname{rhs} \text { of }(6.30)=\operatorname{Det}\left(\mathrm{I}+\sum_{a=1}^{\infty} \mathrm{K}_{a}\right)
$$

where the term $\mathrm{K}_{a}$ in the Fredholm kernel is associated with the transfer matrix $\mathrm{T}_{a}$ :

$$
\mathrm{K}_{a}\left(z_{1}, z_{2}\right)=\frac{1}{2 \pi i} \frac{\mathrm{T}_{a}^{[-a]}(v)}{u-v-i a \epsilon} .
$$

\footnotetext{
${ }^{19}$ In the $\mathfrak{s l}(2)$ case the factor $(-1)^{n}$ in (5.2) is not compensated and should be added in (6.30). This will lead to a factor $(-1)^{a}$ in (6.32), (6.33) and (6.35).
} 
The Fredholm determinant (6.31) can be computed with the help of the explicit expression for the generating function for the transfer matrices, generating function for the transfer matrices, (5.22) for $\mathfrak{s u}(2)$ or $(5.55)$ for $\mathfrak{s l}(2)$. For that we represent the integral operator with kernel (6.32) can be written in a factorised form

$$
\mathrm{K}_{a}=\mathrm{T}_{a}^{[-a]} \mathrm{D}^{-2 a} \mathrm{P}_{-}
$$

where the operator $\mathrm{P}_{-}$, which plays the role of the identity operator, ${ }^{20}$ acts as

$$
\mathrm{P}_{-} \psi(u)=\int_{U_{-}} \frac{d z}{2 \pi i} \frac{\psi(z)}{u-z-i 0} \text {. }
$$

Now the sum in (6.31) turns out to be exactly the generating function for the transfer matrices, multiplied from the right by the operator $\mathrm{P}_{-}$,

$$
\left[\mathcal{C}_{123}^{\bullet \circ o}\right]_{\mathfrak{s u}(2)}^{\text {bottom }} \rightarrow \operatorname{Det}\left(\sum_{a=0}^{\infty} \mathrm{T}_{a}^{[-a]} \mathrm{D}^{-2 a} \mathrm{P}_{-}\right)
$$

Both generating functions are products of operators whose determinants have been already computed in the semiclassical limit $\epsilon \rightarrow 0$. This fact allows us to write the determinant (6.31) as a product of simpler determinants for which we already know the semiclassical limit. (Since the operators act in a functional space, the factorisation to a product of determinants is not exact, but it is corrected by subleading factors.) Let us see that this indeed reproduces the result obtained by clustering.

The $\mathfrak{s u}(2)$ bottom mirror contribution. To evaluate the sum in (6.35), we take the complex conjugate of the generating function (5.22),

$$
\sum_{a=0}^{\infty} \mathrm{T}_{a}^{[-a]} \mathrm{D}^{-2 a}=\left(1-g \mathrm{D}^{-2}\right)\left(1-g f^{-1} \mathrm{D}^{-2}\right)^{-2}\left(1-g f^{-1} \mathrm{D}^{-2} \bar{f}\right) .
$$

where $f=R^{(+)} / R^{(-)}, \bar{f}=B^{(-)} / B^{(+)}$and the function $g(u)$ is defined (5.13). Following the prescription of section 5.1, which is valid at strong coupling, will first take the semiclassical limit of these functions and then continue analytically to the lower edge of the Zhukovsky cut. This amounts to replacing

$$
\sum_{a=0}^{\infty} \mathrm{T}_{a}^{[-a]} \mathrm{D}^{-2 a} \rightarrow\left(1-\bar{Y}^{-1} \mathrm{D}^{-2}\right)\left(1-X^{-1} \mathrm{D}^{-2}\right)^{-2}\left(1-Y^{-1} \mathrm{D}^{-2}\right), \quad u \in U_{-}
$$

\footnotetext{
${ }^{20} \mathrm{Up}$ to exponentially smal terms the operator $\mathrm{P}_{-}$is the identity operator in the space $\mathcal{H}_{-}$of functions analytic in the lower half-plane and decaying exponentially for $x<-1$ or $x>+1$ on the real axis (in the normalization $2 g \epsilon=1$ ).
} 
where

$$
Y(x) \rightarrow e^{i \tilde{p}_{2}(x)+i \tilde{p}_{3}(x)-i \tilde{p}_{1}(x)}, \bar{Y}(x) \rightarrow e^{i \tilde{p}_{1}(1 / x)-i \tilde{p}_{2}(1 / x)-i \tilde{p}_{3}(1 / x)}, X(x) \rightarrow e^{-i p(x) \frac{\Delta_{1}-L_{2}-L_{3}}{2}} .
$$

In the limit we are concerned with, the last factor in (6.33) acts as the identity operator and the product (6.37) is a product of operators of the type (6.9) whose determinants we know how to compute. Since in the leading approximation the determinant of a product of operators equals the product of their determinants, the contribution of the bottom particles is given by the product

$$
\begin{aligned}
{\left[\mathcal{C}_{123}^{\bullet \circ \circ}\right]_{\mathfrak{s u}(2)}^{\text {bottom }} } & \rightarrow \frac{\operatorname{Det}\left(1-\bar{Y}^{-1} \mathrm{D}^{-2}\right) \operatorname{Det}\left(1-Y^{-1} \mathrm{D}^{-2}\right)}{\operatorname{Det}\left(1-X^{-1} \mathrm{D}^{-2}\right) \operatorname{Det}\left(1-X^{-1} \mathrm{D}^{-2}\right)} \\
& \simeq \frac{\operatorname{Det}\left(1-\mathrm{D}^{2} \bar{Y}\right) \operatorname{Det}\left(1-\mathrm{D}^{2} Y\right)}{\operatorname{Det}\left(1-\mathrm{D}^{2} X\right) \operatorname{Det}\left(1-\mathrm{D}^{2} X\right)}
\end{aligned}
$$

Evaluating each of the factors by (6.11), we write

$$
\log \left[\mathcal{C}_{123}^{\bullet \circ \circ}\right]_{\mathfrak{s u}(2)}^{\text {bottom }} \rightarrow \frac{1}{\epsilon} \int_{U_{-}} \frac{d u}{2 \pi}\left(\operatorname{Li}_{2}[Y(x)]+\operatorname{Li}_{2}[\bar{Y}(x)]-2 \operatorname{Li}_{2}[X(x)) .\right.
$$

Using the relations $\bar{Y}(u-i 0)=-1 / Y(u+i 0)$ and $X(u-i 0)=1 / X(u+i 0)$, this linear integral can be further expressed as a contour integral around the Zhukovsky cut (or along the unit circle in the $x$-plane)

$$
\log \left[\mathcal{C}_{123}^{\bullet \circ \circ}\right]_{\mathfrak{s u}(2)}^{\text {bottom }} \rightarrow \frac{1}{\epsilon} \oint_{U} \frac{d u}{2 \pi}\left(\operatorname{Li}_{2}(Y(x))-\operatorname{Li}_{2}(X(x))\right)
$$

which coincides with the semiclassical expression (5.53) obtained by clustering.

We have found that the contribution of the bottom mirror particles is given in the strong coupling, semiclassical limit by the determinant the generating function for the transfer matrices considered as a difference operator:

$$
\left[\mathcal{C}_{123}^{\bullet \bullet \circ}\right]_{\mathfrak{s u}(2)}^{\text {bottom }}=\operatorname{Det}^{-1}\left(\operatorname{sdet}\left(1-G D^{2}\right)\right), \quad G=\operatorname{diag}(X, X \mid Y, \bar{Y})
$$

When the operator $\mathrm{D}^{2}$ is considered as a classical expansion parameter as in (5.29), the superdeterminant in the parenthesis gives the equation of the classical spectral curve $W(z, u) \equiv$ sdet $(z-G(u))=0$. The fact that the spectral curve appears as an operator (sometimes called "quantum spectral curve") is very intriguing and merit to be better understood.

The $\mathfrak{s l}(2)$ bottom mirror contribution. Proceeding in the same way as in the $\mathfrak{s u}(2)$ case, we first write the $\mathfrak{s l}(2)$ analog of (6.29), which differs only by a sign $(-1)^{n}$. Then we 
express the determinant in terms of the generating function for the T-matrices (5.56), which can be written in the form

$$
\sum_{a \geq 0}(-1)^{a} \mathrm{~T}_{a}^{[-a]} \mathrm{D}^{-2 a}=\left(1-g^{-} \mathrm{D}^{-2}\right)^{-1}\left(1-g^{-} \mathrm{D}^{-2} f\right)^{2}\left(1-g^{-} \bar{f}^{-1} \mathrm{D}^{-2} f\right)^{-1}
$$

Evaluating the determinant as in the $\mathfrak{s u}(2)$ case and taking into account the asymptotics (5.17) and (5.21), we obtain an expression identical to (5.53), with the quasimomenta given by $(5.58)$.

There is no principal difficulty in computing the subleading term on the rhs of (6.41), which is of order $\epsilon^{0}$. The subleading comes from the $\epsilon^{2}$ correction to the approximation (5.9), as well as from the subleading term in the expansion of the logarithm of the functional determinant (6.42). The latter will contain diagonal terms of the form (3.39) with $F$ replaced by $X$ and $Y$, as well as a cross term, which represent a correction to the factorised form (6.39) of the determinant.

\section{Conclusion and outlook}

In this paper, we developed two different methods to take the semiclassical limit of threepoint functions in the $\mathfrak{s u}(2)$ and $\mathfrak{s l}(2)$ sectors of $\mathcal{N}=4$ SYM theory. The first method is based on contour deformations of the multiple contour integral representation of the structure constant, which leads to formation of bound states, a phenomena which we call clustering. The second method applies the generalised Fredholm determinant and the Fermi gas approach. Using these methods, we analyse in detail the semiclassical limit of two configurations of three-point functions.

For the type-I-I-II case where all the three operators are non-BPS, we take the semiclassical limit of the asymptotic structure constant at any coupling both for $\mathfrak{s u}(2)$ and $\mathfrak{s l}(2)$ sectors. For the simpler case where two operators are BPS and the third is non-BPS we are able to achieve more. Apart from the semiclassical limit of the asymptotic structure constant, which is a special case of the previous type, we are able to take into account the contribution from all the bottom mirror excitations. The multiple contour integral is considerably more complicated compared to the asymptotic part and we can only take the semiclassical limit at strong coupling. We compare our results of both configurations at strong coupling with the string theoretic calculation and find that they match precisely.

Our work leaves many interesting open questions to be explored in the near future. One immediate problem is to take into account the contributions from mirror excitations on all the three mirror edges in order to obtain a complete result of the structure constant. It 
is known that when the rapidities of two mirror excitations on two different mirror edges coincide, the integrand is divergent. Once these divergences are properly resolved, we can further develop our method and try to reproduce the full result at strong coupling.

The results obtained in this work for asymptotic part are valid at any coupling while for the bottom mirror contribution we only take the semiclassical limit at strong coupling. It is desirable to fix this imbalance by studying in more detail the semiclassical limit for the mirror parts at finite coupling. The Zhukovsky cut disappears at finite coupling since $g^{\prime}=g \epsilon \rightarrow 0$, but it is not clear whether the wrapping correction will disappear as well. We are planning to address this problem elsewhere.

We also restricted our analysis to the simplest set-ups, namely the type-I-I-II case and the BPS-BPS-non BPS case. Results both at strong coupling and weak coupling based on classical integrability techniques strongly suggest that our method can be generalised to more general set-ups, in particular the type-I-I-I case. This is one of the most interesting and at the same time challenging configuration where all the three operators are entangled with each other and the result in general does not factorise into simpler building blocks. At weak coupling, the semiclassical limit was obtained very recently in [21] by using similar methods as those used at strong coupling. Reproducing this result directly from the sum over partitions, both at tree-level and at finite coupling, remains an important open question.

Another direction of generalisation is to consider three-point functions with operators in other sectors, especially those higher rank sectors. The spectral problem has an elegant solution in the semiclassical limit in terms of algebraic curves. Our result in the semiclassical limit shows that the structure constants in this limit also takes a compact form and the dependence of three operators are only through the quasi-momenta. It is tempting to conjecture that this compact form survives for higher rank sectors. If this were the case, we will have an equally elegant description of semiclassical three-point functions in terms of the spectral curves.

It is also important to take into account the subleading corrections in a systematic manner. In our approach, the computation of the subleading corrections is straightforward and is given by the Mayer expansion of the gas of clusters. In the fermionic formalism, the Mayer expansion becomes the standard semiclassical expansion for the bosonised system. It would be interesting to compare our formalism with the Fermi gas approach developed in [19], where the subleading corrections can be worked out by the Wigner-Kirkwood expansion. The efficiency of the two methods can be compared on models which exhibit the clustering phenomenon, but whose subleading corrections (at all order) are nonetheless completely determined by the Wigner-Kirkwood expansion. An example of of such a model is considered in Appendix F. 
We would like to emphasise that we consider the methods developed here as more important than the final results, which just confirm what has been widely expected. First, in our formalism the asymptotic part and the wrapping corrections look very similarly and are evaluated by the same method. Second, we were successful in computing the sum over the mirror particles due to the appearance of the "quantum spectral curve" as finite difference operator whose classical limit is the classical spectral curve. The contribution of the mirror particles is given by the determinant of this operator in the functional space. The fact that the final result depends only on the eigenvalues of the monodromy matrix could give us a hint how to generalise the approach to the whole symmetry group, shortcutting the intimidating sums that appear in the hexagon proposal.

Finally, it is worth mentioning that similar integral representation shows up in the study of stochastic processes. In particular, the integral appears in the resolution of identity. This may provide us with new insights in understanding the origin of integral representation of structure constants in the present work.

\section{Acknowledgements}

The authors are highly indebted to Benjamin Basso for numerous insightful discussions. D.S. would like to thank Andrei Belitsky for discussions concerning the strong coupling limit of the amplitudes, and to Alexei Borodin, Ivan Corwin, Jan de Gier, Alexander Povolotsky and Tomohiro Sasamoto for discussions concerning role of integral representations in the context of stochastic processes. Y.J. would like to thank Zoltan Bajnok and Alessandro Sfondrini for discussions on the analytic structure of the dressing phase. Research at the Perimeter Institute is supported by the Government of Canada through NSERC and by the Province of Ontario through MRI. The research of Y.J. is partially supported by the Swiss National Science Foundation through the NCCR SwissMap. The research of I.K. and D.S. leading to these results has received funding from the People Programme (Marie Curie Actions) of the European Union's Seventh Framework Programme FP7/2007-2013/ under REA Grant Agreement No 317089. I.K. gratefully acknowledges support from the Simons Center for Geometry and Physics, Stony Brook University. D.S. thanks Arizona

State University and KITP Santa Barbara for warm hospitality and acknowledges support by the National Science Foundation under Grant No. NSF PHY11-25915. 


\section{A An example for clustering}

In this appendix, we give an explicit example of clustering. Consider the case $n=3$, we have

$$
\mathrm{I}_{3}=\oint_{\mathcal{C}_{\mathbf{u}}} \frac{d z_{1} d z_{2} d z_{3}}{(2 \pi \epsilon)^{3}} F\left(z_{1}\right) F\left(z_{2}\right) F\left(z_{3}\right) \Delta\left(z_{1}, z_{2}\right) \Delta\left(z_{1}, z_{3}\right) \Delta\left(z_{2}, z_{3}\right)
$$

1. Deform contour of $z_{3}$, we obtain

$$
\begin{aligned}
\mathrm{I}_{3}= & \oint_{\mathcal{C}_{3}} \frac{d z_{3}}{2 \pi \epsilon} \oint_{\mathcal{C}_{\mathbf{u}}} \frac{d z_{1} d z_{2}}{(2 \pi \epsilon)^{2}} F\left(z_{1}\right) F\left(z_{2}\right) F\left(z_{3}\right) \Delta\left(z_{1}, z_{2}\right) \Delta\left(z_{1}, z_{3}\right) \Delta\left(z_{2}, z_{3}\right) \\
& +\frac{1}{2} \oint_{\mathcal{C}_{\mathbf{u}}} \frac{d z_{1} d z_{2}}{(2 \pi \epsilon)^{2}} F\left(z_{1}\right) F_{2}\left(z_{2}\right) \Delta_{12}\left(z_{1}, z_{2}\right) \\
& +\frac{1}{2} \oint_{\mathcal{C}_{\mathbf{u}}} \frac{d z_{1} d z_{2}}{(2 \pi \epsilon)^{2}} F_{2}\left(z_{1}\right) F\left(z_{2}\right) \Delta_{21}\left(z_{1}, z_{2}\right) .
\end{aligned}
$$

2. Deform contour of $x_{2}$ in (A.2), we obtain

$$
\begin{aligned}
\mathrm{I}_{3}= & \oint_{\mathcal{C}_{3}} \frac{d z_{3}}{2 \pi \epsilon} \oint_{\mathcal{C}_{2}} \frac{d z_{2}}{2 \pi \epsilon} \oint_{\mathcal{C}_{\mathbf{u}}} \frac{d z_{1}}{2 \pi \epsilon} F\left(z_{1}\right) F\left(z_{2}\right) F\left(z_{3}\right) \Delta\left(z_{1}, z_{2}\right) \Delta\left(z_{1}, z_{3}\right) \Delta\left(z_{2}, z_{3}\right) \\
& +\frac{1}{2} \oint_{\mathcal{C}_{3}} \frac{d z_{3}}{2 \pi \epsilon} \oint_{\mathcal{C}_{\mathbf{u}}} \frac{d z_{1}}{2 \pi} F_{2}\left(z_{1}\right) F\left(z_{3}\right) \Delta_{21}\left(z_{1}, z_{3}\right) \\
& +\frac{1}{2} \oint_{\mathcal{C}_{2}} \frac{d z_{2}}{2 \pi \epsilon} \oint_{\mathcal{C}_{\mathbf{u}}} \frac{d z_{1}}{2 \pi} F\left(z_{1}\right) F_{2}\left(z_{2}\right) \Delta_{12}\left(z_{1}, z_{2}\right) \\
& +\frac{1}{2} \oint_{\mathcal{C}_{2}} \frac{d z_{2}}{2 \pi \epsilon} \oint_{\mathcal{C}_{\mathbf{u}}} \frac{d z_{1}}{2 \pi i} F_{2}\left(z_{1}\right) F\left(z_{2}\right) \Delta_{21}\left(z_{1}, z_{2}\right) \\
& +2 \times \frac{1}{3} \oint_{\mathcal{C}_{\mathbf{u}}} \frac{d z_{1}}{2 \pi \epsilon} F_{3}\left(z_{1}\right)
\end{aligned}
$$

The three terms containing a double integral are equal, so that we therefore obtain the

multiplicities in figure 3.7. The procedure can be generalised to any number of integrals.

\section{B Scaling limit of the $\mathfrak{s l}(2)$ and $\mathfrak{s u}(2)$ scalar factors}

Here we give the crossing transforms of the scalar factor $h_{\mathfrak{s l}(2)}=h_{D D}$ and $h_{\mathfrak{s u}(2)}=h_{Y Y}$ and their semiclassical limit. We recall first the definitions of the various objects, together with their properties under the crossing transformation,

$$
\begin{aligned}
& h_{\mathfrak{s}(2)}(u, v)=\frac{x^{-}-y^{-}}{x^{-}-y^{+}} \frac{1-\frac{1}{x^{-} y^{+}}}{1-\frac{1}{x^{+} y^{+}}} \frac{1}{\sigma(u, v)}, \\
& h_{\mathfrak{s l}(2)}\left(u^{2 \gamma}, v\right)=\frac{1-\frac{1}{x^{+} y^{-}}}{1-\frac{1}{x^{-} y^{+}}} \sigma(u, v)=\frac{\sigma(u, v)}{A(u, v)},
\end{aligned}
$$




$$
\begin{aligned}
& h_{\mathfrak{s u}(2)}(u, v)=\frac{x^{-}-y^{-}}{x^{+}-y^{-}} \frac{1-\frac{1}{x^{-} y^{+}}}{1-\frac{1}{x^{+} y^{+}}} \frac{1}{\sigma(u, v)}, \\
& h_{\mathfrak{s u}(2)}\left(u^{2 \gamma}, v\right)=\frac{y^{+}}{y^{-}} \sigma(u, v), \quad b_{\mathfrak{s u}(2)}=e^{-i p(v)} h_{\mathfrak{s u}(2)}\left(u^{2 \gamma}, v\right)=\sigma(u, v) .
\end{aligned}
$$

The other transformation properties used are

$$
\begin{aligned}
h_{\mathfrak{s l}(2)}\left(u^{4 \gamma}, v\right) & =1 / h_{\mathfrak{s l}(2)}(v, u) \\
h_{\mathfrak{s l}(2)}\left(u^{2 \gamma}, v^{2 \gamma}\right) & =h_{\mathfrak{s l}(2)}(u, v), \\
h_{\mathfrak{s u}(2)}\left(u^{4 \gamma}, v\right) & =1 / h_{\mathfrak{s u}(2)}(v, u) \\
h_{\mathfrak{s u}(2)}\left(u^{2 \gamma}, v^{2 \gamma}\right) & =e^{i p(u)-i p(v)} h_{\mathfrak{s u}(2)}(u, v) .
\end{aligned}
$$

The semiclassical limit $\epsilon \rightarrow 0$ of the various quantities is

$$
\begin{aligned}
& \log h_{\mathfrak{s u}(2)}(u, v) \rightarrow-\frac{i\left(x^{2} y^{2}-x^{2}+x y-y^{2}\right)}{g\left(x^{2}-1\right)\left(y^{2}-1\right)(x-y)} \\
&=-\frac{i \epsilon y^{\prime}}{x-y}+\frac{i p(x)}{y^{2}-1}=\frac{i \epsilon x^{\prime}}{y-x}-\frac{i p(y)}{x^{2}-1}, \\
& \log b_{\mathfrak{s u}(2)}(u, v)= \log \sigma(u, v) \\
& \rightarrow \log h_{\mathfrak{s u}(2)}(1 / x, y)-i p(y)=\log h_{\mathfrak{s u}(2)}(x, 1 / y)+i p(x) \\
&=-\frac{i \epsilon y^{\prime}}{1 / x-y}-\frac{i p(x)}{y^{2}-1}-i p(y)=\frac{i \epsilon x^{\prime}}{1 / y-x}+\frac{i p(y)}{x^{2}-1}+i p(x) \\
& \log h_{\mathfrak{s l}(2)}(u, v) \rightarrow \frac{i x y(x y-1)}{g\left(x^{2}-1\right)\left(y^{2}-1\right)(x-y)} \frac{i \epsilon y^{\prime}}{x-y}-\frac{i p(y)}{x^{2}-1}=\frac{i \epsilon x^{\prime}}{x-y}+\frac{i p(x)}{y^{2}-1}, \\
& \log b_{\mathfrak{s l}(2)}(u, v) \rightarrow \frac{\log h_{\mathfrak{s l}(2)}(1 / x, y)}{1 / x-y}-\frac{i p(y)}{1 / x^{2}-1}=\frac{i \epsilon x^{\prime}}{x-1 / y}-\frac{i p(x)}{1-1 / y^{2}} .
\end{aligned}
$$

\section{Computing the phase factor for the asymptotic $C_{123}^{\bullet \bullet}$}

In this appendix, we compute the phase factor denoted by phase in (4.5) in the main text and show explicitly how to derive (4.8) from (4.5). This phase factor comes from changing between spin chain and string frames when we perform crossing transformations. The phase factor is trivial for $\mathfrak{s l}(2)$ case but is non-trivial for the $\mathfrak{s u}(2)$ case. In what follows, it is convenient to write the hexagon form factor in the form

$$
\mathrm{H}\left(\alpha_{1}\left|\alpha_{3}\right| \alpha_{2}\right)=\left\langle\mathfrak{h} \mid \alpha_{1}\right\rangle\left|\alpha_{2}\right\rangle\left|\alpha_{3}\right\rangle .
$$


The excitations in the two frames are related by

$$
\mathcal{D}_{\text {string }}=\mathcal{D}_{\text {spin }}, \quad \Phi_{\text {string }}=\sqrt{Z} \Phi_{\text {spin }} \sqrt{Z} .
$$

We see that the derivative excitation is the same in both frames which is the reason that the phase factor is trivial. A state with $n$ scalar excitations in the two frames are related by

$$
\begin{aligned}
\left|\Phi_{1} \cdots \Phi_{n}\right\rangle_{\text {string }} & =\left|\sqrt{Z} \Phi_{1} Z \Phi_{2} Z \cdots Z \Phi_{n} \sqrt{Z}\right\rangle_{\text {spin }} \\
& =F_{n}\left|Z^{n} \Phi_{1} \cdots \Phi_{n}\right\rangle_{\text {spin }}
\end{aligned}
$$

where in the second line we have moved all the $Z$-markers to the leftmost by the rule

$$
\left|\cdots \Phi_{k} Z^{a} \cdots\right\rangle=e^{i p_{k} a}\left|\cdots Z^{a} \Phi_{k} \cdots\right\rangle .
$$

It is straightforward to derive that

$$
F_{n}=\prod_{j=1}^{n} \frac{e^{i p_{j} / 2} \zeta_{j}}{\zeta}, \quad \zeta_{k}=e^{i p_{k}} \zeta_{k-1}, \quad \zeta_{1}=\zeta
$$

We can also write (C.3) as

$$
\left|\Phi_{1} \cdots \Phi_{n}\right\rangle_{\mathrm{spin}}=F_{n}^{-1}\left|Z^{-n} \Phi_{1} \cdots \Phi_{n}\right\rangle_{\mathrm{string}} .
$$

Substituting (C.6) into the hexagon form factor (C.1)

$$
\left\langle\mathfrak{h} \mid \alpha_{1}\right\rangle\left|\alpha_{2}\right\rangle\left|\alpha_{3}\right\rangle_{\text {spin }}=\left(F_{\alpha_{1}} F_{\alpha_{2}} F_{\alpha_{3}}\right)^{-1}\left\langle\mathfrak{h} \mid Z^{-\left|\alpha_{1}\right|} \alpha_{1}\right\rangle\left|Z^{-\left|\alpha_{2}\right|} \alpha_{2}\right\rangle\left|Z^{-\left|\alpha_{3}\right|} \alpha_{3}\right\rangle_{\text {string }} .
$$

The next step is to move all the excitations on the same edge and then pull out the $Z$ makers by the rule

$$
\left\langle\mathfrak{h} \mid Z^{n} \psi\right\rangle=z^{n}\langle\mathfrak{h} \mid \psi\rangle, \quad z=e^{-i P / 2},
$$

where $P$ is the total momentum of the state $|\psi\rangle$. This leads to

$$
\begin{aligned}
\left\langle\mathfrak{h} \mid \alpha_{1}\right\rangle\left|\alpha_{2}\right\rangle\left|\alpha_{3}\right\rangle_{\text {spin }}= & e^{\frac{i}{2}\left[p\left(\alpha_{1}\right)\left(\left|\alpha_{1}\right|+\left|\alpha_{3}\right|-\left|\alpha_{2}\right|\right)+p\left(\alpha_{2}\right)\left(\left|\alpha_{2}\right|+\left|\alpha_{1}\right|-\left|\alpha_{3}\right|\right)+p\left(\alpha_{3}\right)\left(\left|\alpha_{3}\right|+\left|\alpha_{2}\right|-\left|\alpha_{1}\right|\right)\right]} \\
& \times\left(F_{\alpha_{1}} F_{\alpha_{2}} F_{\alpha_{3}}\right)^{-1}\left\langle\mathfrak{h} \mid \alpha_{1}\right\rangle\left|\alpha_{2}\right\rangle\left|\alpha_{3}\right\rangle_{\text {string }},
\end{aligned}
$$

where $\left|\alpha_{1}\right|,\left|\alpha_{2}\right|,\left|\alpha_{3}\right|$ denote the cardinality and $p\left(\alpha_{1}\right), p\left(\alpha_{2}\right), p\left(\alpha_{3}\right)$ denote the total momenta of the sets $\alpha_{1}, \alpha_{2}$ and $\alpha_{3}$ respectively. Now that we are in the string frame, we can perform the crossing transformation and obtain the fundamental hexagon form factor where all excitations are on the same edge

$$
\left\langle\mathfrak{h} \mid \alpha_{1}\right\rangle\left|\alpha_{2}\right\rangle\left|\alpha_{3}\right\rangle_{\text {string }}=(-1)^{\left|\alpha_{3}\right|}\left\langle\mathfrak{h} \mid \alpha_{1}^{4 \gamma}, \alpha_{3}^{2 \gamma}, \alpha_{2}\right\rangle_{\text {string }} .
$$


The factor $(-1)^{\left|\alpha_{3}\right|}$ appears due to crossing transformation $\left(\Phi^{a \dot{b}}\right)^{2 \gamma} \rightarrow-\Phi^{b \dot{a}}$.

In order to compute the fundamental hexagon form factor, we need to change back to the spin chain frame. We apply the rules (C.2) and (C.4) again

$$
\begin{aligned}
\left\langle\mathfrak{h} \mid \alpha_{1}^{4 \gamma}, \alpha_{3}^{2 \gamma}, \alpha_{2}\right\rangle_{\text {string }}= & F_{\alpha_{1}} F_{\alpha_{2}} F_{\alpha_{3}}^{-1}\left\langle\mathfrak{h} \mid Z^{\left|\alpha_{1}\right|} \alpha_{1}^{4 \gamma}, Z^{\left|\alpha_{3}\right|} \alpha_{3}^{2 \gamma}, Z^{\left|\alpha_{2}\right|} \alpha_{2}\right\rangle_{\text {spin }}, \quad \text { (C.11) } \\
= & F_{\alpha_{1}} F_{\alpha_{2}} F_{\alpha_{3}}^{-1} e^{i\left|\alpha_{2}\right|\left(-p\left(\alpha_{3}\right)+p\left(\alpha_{1}\right)\right)+i\left|\alpha_{3}\right| p\left(\alpha_{1}\right)-\frac{i}{2}\left(\left|\alpha_{1}\right|+\left|\alpha_{2}\right|+\left|\alpha_{3}\right|\right)\left(p\left(\alpha_{1}\right)-p\left(\alpha_{3}\right)+p\left(\alpha_{2}\right)\right)} \\
& \times\left\langle\mathfrak{h} \mid \alpha_{1}^{4 \gamma}, \alpha_{3}^{2 \gamma}, \alpha_{2}\right\rangle_{\text {spin }},
\end{aligned}
$$

where in the first line, we move the $Z$-markers corresponds to the excitations $\alpha_{1}, \alpha_{2}$ and $\alpha_{3}$ to the leftmost of the corresponding set, which gives rise to the factors $F_{\alpha_{1}}, F_{\alpha_{2}}$ and $F_{\alpha_{3}}^{-1}=F_{\alpha_{3}^{2 \gamma}}$. In the second line, we further move the $Z$-markers $Z^{\left|\alpha_{1}\right|}, Z^{\left|\alpha_{2}\right|}$ and $Z^{\left|\alpha_{3}\right|}$ to the leftmost and then pull out the $Z$-markers by the rule (C.8). Note that $p\left(\alpha_{3}\right)^{2 \gamma}=-p\left(\alpha_{3}\right)$.

Combining all the phase factors from (C.9), (C.10) and (C.11)

$$
\left\langle\mathfrak{h} \mid \alpha_{1}\right\rangle\left|\alpha_{2}\right\rangle\left|\alpha_{3}\right\rangle_{\text {spin }}=(-1)^{\left|\alpha_{3}\right|} F_{\alpha_{3}}^{-2} e^{i\left|\alpha_{3}\right|\left(p\left(\alpha_{1}\right)-p\left(\alpha_{2}\right)+p\left(\alpha_{3}\right)\right)}\left\langle\mathfrak{h} \mid \alpha_{1}^{4 \gamma}, \alpha_{3}^{2 \gamma}, \alpha_{2}\right\rangle_{\text {spin }} .
$$

Therefore the phase factors in (4.4) read

$$
\begin{aligned}
& \text { phase }_{1}=(-1)^{\left|\alpha_{3}\right|} F_{\alpha_{3}}^{-2} e^{i\left|\alpha_{3}\right|\left(p\left(\alpha_{1}\right)-p\left(\alpha_{2}\right)+p\left(\alpha_{3}\right)\right)}, \\
& \text { phase }_{2}=(-1)^{\left|\bar{\alpha}_{3}\right|} F_{\bar{\alpha}_{3}}^{-2} e^{i\left|\bar{\alpha}_{3}\right|\left(p\left(\bar{\alpha}_{2}\right)-p\left(\bar{\alpha}_{1}\right)+p\left(\bar{\alpha}_{3}\right)\right)} .
\end{aligned}
$$

The total phase factor phase $=$ phase $_{1} \times$ phase $_{2}$. This ends the derivation of the phase factor.

In order to obtain the more compact result (4.8), we need to combine the phase factor (C.13) with the other phase factors which comes from the crossing transformations of the scalar factors of the $\mathfrak{s u}(2)$ sector. There are two sources of the other phase factors. The first source comes from passing from (4.5) to (2.3), where we extract the global factor $(-1)^{N} h^{<}\left(\mathbf{u}_{1}, \mathbf{u}_{1}\right) h^{<}\left(\mathbf{u}_{2}, \mathbf{u}_{2}\right) h^{<}\left(\mathbf{u}_{3}, \mathbf{u}_{3}\right)$ and neglect it systematically. In order to extract this global factor, we used the crossing relation $h_{\mathfrak{s u}(2)}\left(u^{2 \gamma}, v^{2 \gamma}\right)=e^{i p(u)-i p(v)} h_{\mathfrak{s u}(2)}(u, v)$ which leads to

$$
\begin{aligned}
& h_{\mathfrak{s u}(2)}^{<}\left(\alpha_{3}^{2 \gamma}, \alpha_{3}^{2 \gamma}\right)=\text { phase }_{\alpha_{3}} h_{\mathfrak{s u}(2)}^{<}\left(\alpha_{3}, \alpha_{3}\right), \\
& h_{\mathfrak{s u}(2)}^{<}\left(\bar{\alpha}_{3}^{2 \gamma}, \bar{\alpha}_{3}^{2 \gamma}\right)=\text { phase }_{\bar{\alpha}_{3}} h_{\mathfrak{s u}(2)}^{<}\left(\bar{\alpha}_{3}, \bar{\alpha}_{3}\right),
\end{aligned}
$$

with

$$
\text { phase }_{\alpha_{3}}=\prod_{\substack{j, k \in \alpha_{3} \\ j<k}} e^{i p_{j}-i p_{k}}, \quad \text { phase }_{\bar{\alpha}_{3}}=\prod_{\substack{j, k \in \bar{\alpha}_{3} \\ j<k}} e^{i p_{j}-i p_{k}}
$$


Notice that for $\mathfrak{s l}(2)$ sector we have $h\left(u^{2 \gamma}, v^{2 \gamma}\right)=h(u, v)$ and the phase factor is again trivial.

The second source of phase factor comes from rewriting the following terms in (4.5)

$$
\begin{aligned}
& h_{\mathfrak{s u}(2)}\left(\alpha_{1}^{4 \gamma}, \alpha_{3}^{2 \gamma}\right) h_{\mathfrak{s u}(2)}\left(\alpha_{3}^{2 \gamma}, \alpha_{2}\right)=\frac{h_{\mathfrak{s u}(2)}\left(\alpha_{3}^{2 \gamma}, \alpha_{2}\right)}{h_{\mathfrak{s u}(2)}\left(\alpha_{3}^{2 \gamma}, \alpha_{1}\right)}=e^{i\left(p\left(\alpha_{2}\right)-p\left(\alpha_{1}\right)\right)\left|\alpha_{3}\right|} \frac{\sigma\left(\alpha_{3}, \alpha_{2}\right)}{\sigma\left(\alpha_{3}, \alpha_{1}\right)}, \\
& h_{\mathfrak{s u}(2)}\left(\bar{\alpha}_{2}^{4 \gamma}, \bar{\alpha}_{3}^{2 \gamma}\right) h_{\mathfrak{s u}(2)}\left(\bar{\alpha}_{3}^{2 \gamma}, \bar{\alpha}_{1}\right)=\frac{h_{\mathfrak{s u}(2)}\left(\bar{\alpha}_{3}^{2 \gamma}, \bar{\alpha}_{1}\right)}{h_{\mathfrak{s u}(2)}\left(\bar{\alpha}_{3}^{2 \gamma}, \bar{\alpha}_{2}\right)}=e^{i\left(p\left(\bar{\alpha}_{1}\right)-p\left(\bar{\alpha}_{2}\right)\right)\left|\bar{\alpha}_{3}\right|} \frac{\sigma\left(\bar{\alpha}_{3}, \bar{\alpha}_{1}\right)}{\sigma\left(\bar{\alpha}_{3}, \bar{\alpha}_{2}\right)}
\end{aligned}
$$

where we have used the crossing property $h_{\mathfrak{s u}(2)}\left(u^{2 \gamma}, v\right)=e^{i p(v)} \sigma(u, v)$. The phase factors in (C.15) and (C.16) combines nicely with (C.13)

$$
\text { phase }_{1} \times \text { phase }_{2} \times \text { phase }_{\alpha_{3}} \times \text { phase }_{\bar{\alpha}_{3}} \times e^{i\left(p\left(\alpha_{2}\right)-p\left(\alpha_{1}\right)\right)\left|\alpha_{3}\right|} \times e^{i\left(p\left(\bar{\alpha}_{1}\right)-p\left(\bar{\alpha}_{2}\right)\right)\left|\bar{\alpha}_{3}\right|}=1 .
$$

The final result can be written neatly

$$
\mathcal{C}_{123}^{\bullet \bullet \bullet}=\sum_{\alpha_{i} \cup \bar{\alpha}_{i}=\mathbf{u}_{i}} \prod_{i=1}^{3}(-1)^{\left|\bar{\alpha}_{i}\right|} \frac{e^{i p_{\bar{\alpha}_{i}} l_{i-1, i}}}{h_{\mathfrak{s u}(2)}\left(\alpha_{i}, \bar{\alpha}_{i}\right)} \times \frac{1}{h_{\mathfrak{s u}(2)}\left(\alpha_{2}, \alpha_{1}\right) h_{\mathfrak{s u}(2)}\left(\bar{\alpha}_{1}, \bar{\alpha}_{2}\right)} \frac{\sigma\left(\alpha_{3}, \alpha_{2}\right)}{\sigma\left(\alpha_{3}, \alpha_{1}\right)} \frac{\sigma\left(\bar{\alpha}_{3}, \bar{\alpha}_{1}\right)}{\sigma\left(\bar{\alpha}_{3}, \bar{\alpha}_{1}\right)}
$$

which is (4.8). For completeness, we also give the result for the $\mathfrak{s l}(2)$ sector which takes a very similar form

$$
\mathcal{C}_{123}^{\bullet \bullet \bullet}=\sum_{\alpha_{i} \cup \bar{\alpha}_{i}=\mathbf{u}_{i}} \prod_{i=1}^{3}(-1)^{\mid \bar{\alpha}_{i} i} \frac{e^{i p_{\bar{\alpha}_{i}} l_{i-1, i}}}{h\left(\alpha_{i}, \bar{\alpha}_{i}\right)} \times \frac{1}{h\left(\alpha_{2}, \alpha_{1}\right) h\left(\bar{\alpha}_{1}, \bar{\alpha}_{2}\right)} \frac{h\left(\alpha_{3}^{2 \gamma}, \alpha_{2}\right)}{h\left(\alpha_{3}^{2 \gamma}, \alpha_{1}\right)} \frac{h\left(\bar{\alpha}_{3}^{2 \gamma}, \bar{\alpha}_{1}\right)}{h\left(\bar{\alpha}_{3}^{2 \gamma}, \bar{\alpha}_{2}\right)} .
$$

\section{The analytic structure of the transfer matrices in mirror kinematics}

The purpose of this appendix is to show that the combination of the dressing phase with the extra factors from (5.15),

$$
\sigma_{a 1}(u, \mathbf{u}) \prod_{k=1}^{a-1} \frac{R^{(-)[a-2 k]}}{R^{(+)[a-2 k]}}(u)
$$

does not have any cut between the cuts $x^{[ \pm a]}$ when continued to the mirror dynamics in the variable $u$. To do so, we compute the jump of the logarithm of the function given above, between $u=u_{0}-i \epsilon(a / 2-k)+i 0$ and $u=u_{0}-i \epsilon(a / 2-k)-i 0$ on the interval $u_{0} \in[-1,1]^{21}$. $x^{[a-2 k]}(u)$ has a second order branch cut and we call $x^{[a-2 k]}$ the determination $u_{0}+i 0$ and

\footnotetext{
${ }^{21}$ We choose $\epsilon=1 / 2 g$ for simplicity.
} 
$1 / x^{[a-2 k]}$ the determination $u_{0}-i 0$. It is clear that the jump of the logarithm of the product part is given by

$$
\log \left(\frac{x^{[a-2 k]}-\mathbf{y}^{+}}{x^{[a-2 k]}-\mathbf{y}^{-}} \frac{1 / x^{[a-2 k]}-\mathbf{y}^{-}}{1 / x^{[a-2 k]}-\mathbf{y}^{+}}\right) .
$$

To compute the jump of the dressing phase we use the DHM representation [38].

DHM representation. The dressing phase has the following structure:

$$
\log \sigma(u, v)=i\left(\chi\left(x^{+}, y^{-}\right)-\chi\left(x^{-}, y^{-}\right)-\chi\left(x^{+}, y^{+}\right)+\chi\left(x^{-}, y^{+}\right)\right) .
$$

For two bound states of size $a$ and $b$, it is given by

$$
\log \sigma_{a b}(u, v)=i\left(\chi\left(x^{[a]}, y^{[-b]}\right)-\chi\left(x^{[-a]}, y^{[-b]}\right)-\chi\left(x^{[a]}, y^{[b]}\right)+\chi\left(x^{[-a]}, y^{[b]}\right)\right)
$$

The function $\chi(x, y)$ is given by the following integral expression first obtained by Dorey, Hofman and Maldacena [38]

$$
\chi(x, y)=-i \oint_{|z|=1} \frac{d z}{2 \pi i} \oint_{|w|=1} \frac{d w}{2 \pi i} \frac{1}{x-z} \frac{1}{y-w} \log \frac{\Gamma\left[1+i g\left(z+\frac{1}{z}-w-\frac{1}{w}\right)\right]}{\Gamma\left[1-i g\left(z+\frac{1}{z}-w-\frac{1}{w}\right)\right]} .
$$

This expression is valid in the physical kinematics; namely when $|x|>1$ and $|y|>1$. If we want to compute the quantities in the mirror kinematics, we need to analytically continue this expression, for example to the regime $|x|<1$. Under the analytic continuation, the poles of the integrand cross the unit circle and yield extra contribution. For instance, the dressing phase $\sigma_{a b}\left(u^{\gamma}, v\right)$ in the mirror-physical kinematics has extra contributions coming from the analytic continuation of $\chi\left(x^{[a]}, y^{[-b]}\right)-\chi\left(x^{[a]}, y^{[b]}\right)$. The other terms are unaffected.

Analytic continuation. Let us explicitly perform such analytic continuation. More precisely, we analytically continue $x$ in (D.5) to $|x|<1$. In the process, the integral picks up a pole from $1 /(x-z)$. The expression after the analytic continuation is

$$
\chi_{\text {mirror }}(x, y)=\chi_{0}(x, y)+\chi_{\text {jump }}(x, y), \quad|x|<1
$$

where $\chi_{0}$ and $\chi_{\text {jump }}$ are given by

$$
\begin{aligned}
\chi_{0}(x, y) & =-i \oint_{|z|=1} \frac{d z}{2 \pi i} \oint_{|w|=1} \frac{d w}{2 \pi i} \frac{1}{x-z} \frac{1}{y-w} \log \frac{\Gamma\left[1+i g\left(z+\frac{1}{z}-w-\frac{1}{w}\right)\right]}{\Gamma\left[1-i g\left(z+\frac{1}{z}-w-\frac{1}{w}\right)\right]}, \\
\chi_{\text {jump }}(x, y) & =-i \oint_{|w|=1} \frac{d w}{2 \pi i} \frac{1}{y-w} \log \frac{\Gamma\left[1+i g\left(x+\frac{1}{x}-w-\frac{1}{w}\right)\right]}{\Gamma\left[1-i g\left(x+\frac{1}{x}-w-\frac{1}{w}\right)\right]} .
\end{aligned}
$$

$\chi_{0}$ has no singularity as a function of $x$ except the one at $|x|=1$. As we show below, the function $\chi_{\text {jump }}(x, y)$ contains an infinite arrays of branch cuts, which show up in the mirror dynamics of the bound states dressing phase. 

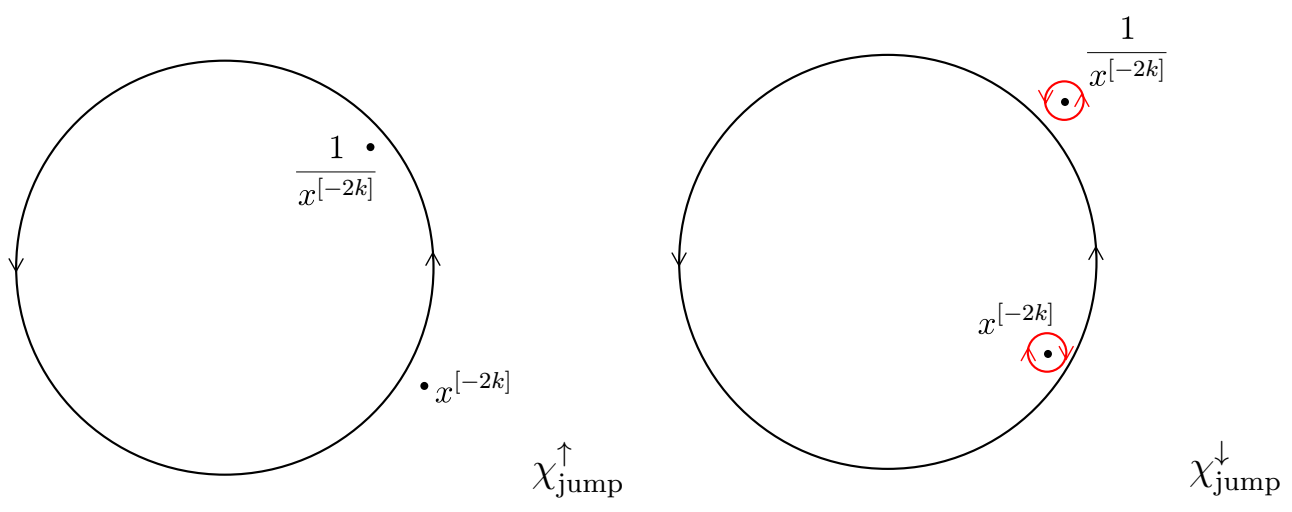

Figure D.9: The integration contours for $\chi_{\text {jump }}^{\uparrow}$ and $\chi_{\text {jump }}^{\downarrow}$ (both denoted in black). For $\chi_{\text {jump }}^{\uparrow}$, $x^{[-2 k]}$ is outside the integration contour whereas $1 / x^{[-2 k]}$ is outside. On the other hand, the situation is opposite for $\chi_{\text {jump }}^{\downarrow}$. The discontinuity $\chi_{\text {jump }}^{\uparrow}-\chi_{\text {jump }}^{\downarrow}$ is given by the integrations around $x^{[-2 k]}$ and $1 / x^{[-2 k]}$, which are denoted in red.

Analytic structure of $\chi_{\text {jump }}$. Let us now study the analytic structure of $\chi_{\text {jump }}$. For this purpose, it is useful to re-express (D.8) by integration by parts as

$$
\begin{aligned}
\chi_{\text {jump }}(x, y) & =-g \oint_{|w|=1} \frac{d w}{2 \pi i} \log (w-y)\left(1-\frac{1}{w^{2}}\right) \\
& \times\left[\Psi\left(1+i g\left(x+\frac{1}{x}-w-\frac{1}{w}\right)\right)+\Psi\left(1-i g\left(x+\frac{1}{x}-w-\frac{1}{w}\right)\right)\right],
\end{aligned}
$$

where

$$
\Psi(z+1)=\frac{d \log \Gamma(z+1)}{d z}=-\sum_{k \geq 1}\left(\frac{1}{z+k}-\frac{1}{k}\right)-\gamma_{E}
$$

This enables us to write, ignoring the constant in the function $\Psi(z)$,

$$
\begin{aligned}
\chi_{\text {jump }}(x, y) & =i \oint_{|w|=1} \frac{d w}{2 \pi i} \log (w-y)\left(1-\frac{1}{w^{2}}\right) \\
& \times \sum_{k \geq 1}\left[\frac{1}{w+\frac{1}{w}-x^{[-2 k]}-\frac{1}{x^{[-2 k]}}}-\frac{1}{w+\frac{1}{w}-x^{[2 k]}-\frac{1}{x^{[2 k]}}}\right] \\
& =i \oint_{|w|=1} \frac{d w}{2 \pi i} \log (w-y) \sum_{k \neq 0} \operatorname{sgn}(k)\left[\frac{x^{[-2 k]}}{w\left(w-x^{[-2 k]}\right)}+\frac{1}{w-1 / x^{[-2 k]}}\right] .
\end{aligned}
$$

Obviously, $\chi_{\text {jump }}(x, y)$, as a function of $x$, has discontinuities whenever one of the poles in the sum above hits the contour of integration $|w|=1$. This happens whenever $x^{[2 k]}$ with $k$ a non-zero integer hits the unit circle. These are exactly the discontinuities we are looking 
for. Moreover, we are interested in the discontinuities of $\chi_{\text {jump }}\left(x^{[a]}, y\right)$ situated between $u_{0}+i a \epsilon / 2$ and $u_{0}-i a \epsilon / 2$. This means that we restrain ourselves to the terms with positive $k, k=1, \ldots, a-1$ in the last line of (D.11).

Let us now compute the discontinuity $\chi_{\text {jump }}^{\uparrow}-\chi_{\text {jump }}^{\downarrow}$ when $x^{[-2 k]}$ crosses the unit circle from going from outside to inside (see figure D.9). In the same time, the pole $1 / x^{[-2 k]}$ crosses the unit circle from inside to outside. The discontinuity is accounted for by the contribution of the two poles,

$$
\begin{aligned}
& \left.\chi^{\uparrow \downarrow}\right|_{u+i k \epsilon}(x, y) \equiv \chi_{\text {jump }}^{\uparrow}-\chi_{\text {jump }}^{\downarrow} \\
& =i\left(\oint_{1 / x^{[-2 k]}}-\oint_{x^{[-2 k]}}\right) \frac{d w}{2 \pi i} \log (w-y)\left[\frac{x^{[-2 k]}}{w\left(w-x^{[-2 k]}\right)}+\frac{1}{w-1 / x^{[-2 k]}}\right]=i \log \frac{1 / x^{[-2 k]}-y}{x^{[-2 k]}-y} .
\end{aligned}
$$

The small subtlety that, after crossing the unit circle, $x^{[-2 k]}$ gets exchanged with $1 / \bar{x}^{[-2 k]}$ instead of $1 / x^{[-2 k]}$ is resolved by noticing that $\chi_{\text {jump }}$ only depends on $x+1 / x$, which on the unit circle is the same as its complex conjugate $\bar{x}+1 / \bar{x}$. Combining everything, we obtain the discontinuity of the mirror dressing factor,

$$
\begin{aligned}
\left.\log \sigma_{a 1}^{\uparrow \downarrow}(x, \mathbf{y})\right|_{u-i(a / 2-k) \epsilon} & =\left.i\left(\chi^{\uparrow \downarrow}\left(x^{[a]}, y^{-}\right)-\chi^{\uparrow \downarrow}\left(x^{[a]}, y^{+}\right)\right)\right|_{u-i(a / 2-k) \epsilon} \\
& =\log \frac{1 / x^{[a-2 k]}-\mathbf{y}^{+}}{x^{[a-2 k]}-\mathbf{y}^{+}} \frac{x^{[a-2 k]}-\mathbf{y}^{-}}{1 / x^{[a-2 k]}-\mathbf{y}^{-}}
\end{aligned}
$$

which exactly compensates the one from (D.2). This completes the proof that the combination in (D.1) does not have any discontinuities in the desired region of the mirror regime.

\section{E The term $\mathrm{B}_{3}$ at strong coupling}

In this appendix we compute the terms $\mathrm{B}_{3}$

$$
\begin{aligned}
\mathrm{B}_{3} & =\frac{1}{3 !} \int \prod_{i=1}^{3} \frac{d z_{i}}{2 \pi \epsilon} \mathrm{T}_{1}\left(z_{i}\right) \Delta\left(z_{1}, z_{2}\right) \Delta\left(z_{1}, z_{3}\right) \Delta\left(z_{2}, z_{3}\right) \\
& +\frac{1}{2} \int \frac{d z_{1} d z_{2}}{(2 \pi \epsilon)^{2}} \mathrm{~T}_{1}\left(z_{1}\right) \mathrm{T}_{2}\left(z_{2}\right) \Delta_{1,2}\left(z_{1}, z_{2}+i \epsilon / 2\right)+\frac{1}{3} \int \frac{d z}{2 \pi \epsilon} \mathrm{T}_{3}(z) .
\end{aligned}
$$

To compute the first term of (E.1), we can recycle the result of the asymptotic integral $\mathrm{I}_{3}$ in appendix A. The combinatorics is the same, with the exception of signs, since in the present case every fusion of two already existing clusters generates a minus sign, $c f$. (5.32) and (5.33). This gives

$$
\frac{1}{3 !}\left(\int \frac{d z}{2 \pi \epsilon} \mathrm{T}_{1}(z)\right)^{3}-\frac{1}{4}\left(\int \frac{d z}{2 \pi \epsilon} \mathrm{T}_{1}(z)\right)\left(\int \frac{d z}{2 \pi \epsilon} \mathrm{T}_{1}(z)^{2}\right)+\frac{1}{9} \int \frac{d z}{2 \pi \epsilon} \mathrm{T}_{1}(z)^{3}
$$


For the second term of (E.1), it is easy to obtain

$$
\frac{1}{2}\left(\int \frac{d z}{2 \pi \epsilon} \mathrm{T}_{1}(z)\right)\left(\int \frac{d z}{2 \pi \epsilon} \mathrm{T}_{2}(z)\right)-\frac{1}{3} \int \frac{d z}{2 \pi \epsilon} \mathrm{T}_{1}(z) \mathrm{T}_{2}(z)
$$

Substituting (E.2) and (E.3) into (E.1), and using (5.28) we obtain as announced

$$
\mathrm{B}_{3}=\frac{1}{3 !}\left(\int \frac{d z}{2 \pi \epsilon} \mathrm{t}_{1}(z)\right)^{3}+\frac{1}{4}\left(\int \frac{d z}{2 \pi \epsilon} \mathrm{t}_{1}(z)\right)\left(\int \frac{d z}{2 \pi \epsilon} \mathrm{t}_{2}(z)\right)+\frac{1}{9} \int \frac{d z}{2 \pi \epsilon} \mathrm{t}_{3}(z) .
$$

\section{F The ABJM matrix model and clustering}

In this Appendix, we re-derive the M-theoretic large $N$ limit of the ABJM matrix model as an extra example of the utility of the clustering method explained in the main text.

The partition function of the $U(N) \times U(N)$ ABJM theory on $S^{3}$ can be computed by the localisation [57]. The result reads

$$
Z(N)=\frac{1}{N !} \int \prod_{i} \frac{d x_{i}}{2 \pi k} \frac{1}{2 \cosh \frac{x_{i}}{2}} \operatorname{det}\left(\frac{1}{2 \cosh \frac{x_{i}-x_{j}}{2 k}}\right)
$$

where $k$ is the Chern-Simons level. To study the M-theory limit $(N \rightarrow \infty, k$ fixed), it is convenient to consider the grand-canonical partition function,

$$
\Theta(z) \equiv \sum_{N} z^{N} Z(N)
$$

where $z$ is the fugacity. As explained in [19], this can be expressed as a Fredholm determinant

$$
\Theta(z)=\operatorname{det}(1+z \hat{\rho})=\exp \left(-\sum_{n=1}^{\infty} \frac{(-z)^{n}}{n} \operatorname{Tr}\left(\hat{\rho}^{n}\right)\right) .
$$

Here the action of the operator $\hat{\rho}$ and the spectral trace $\operatorname{Tr}\left(\hat{\rho}^{n}\right)$ are given by

$$
\begin{aligned}
\hat{\rho} \cdot f(x) & =\int \frac{d y}{2 \pi k} \rho(x, y) f(y), \\
\operatorname{Tr}\left(\hat{\rho}^{n}\right) & =\int \frac{d^{n} x}{(2 \pi k)^{n}} \rho\left(x_{1}, x_{2}\right) \cdots \rho\left(x_{n-1}, x_{n}\right) \rho\left(x_{n}, x_{1}\right),
\end{aligned}
$$

with

$$
\rho(x, y)=\frac{1}{2 \cosh \frac{x}{2}} \frac{1}{2 \cosh \frac{x-y}{2 k}} .
$$


To understand the M-theoretic large $N$ limit, we should understand the small $k$ expansion of the grand canonical partition function $\Theta$. Just as in the case of the three-point function, the kernel $\rho(x, y)$ develops a delta-function singularity in the $k \rightarrow 0$ limit and the integral exhibits the clustering. To see this, we first express the interaction term in the kernel as a sum over rational functions:

$$
\frac{1}{\cosh \frac{x-y}{2 k}}=\sum_{a=0}^{\infty} \frac{4 k^{2} \pi(-1)^{a}(a+1 / 2)}{(x-y)^{2}+4 k^{2} \pi^{2}(a+1 / 2)^{2}} .
$$

This summation over positive integer is reminiscent of the summation over the bound states appearing in the main text. To make clear the relation, we introduce the "bound-state kernel"

$$
\hat{\rho}_{a}=\hat{\rho}_{a+}+\hat{\rho}_{a-},
$$

with

$$
\begin{aligned}
& \hat{\rho}_{a+} \cdot f \equiv \int \frac{d y}{4 \pi \cosh \frac{y}{2}} \frac{i}{(x-y)+2 i k \pi(a+1 / 2)} f(y), \\
& \hat{\rho}_{a-} \cdot f \equiv \int \frac{d y}{4 \pi \cosh \frac{y}{2}} \frac{-i}{(x-y)-2 i k \pi(a+1 / 2)} f(y) .
\end{aligned}
$$

Then, the grand canonical partition function takes a form similar to (6.31),

$$
\ln \Theta=\ln \operatorname{det}\left[1+z\left(\sum_{a}(-1)^{a} \hat{\rho}_{a}\right)\right]=\sum_{n=1}^{\infty} \frac{(-1)^{n-1}}{n} z^{n} \operatorname{Tr}\left[\left(\sum_{a}(-1)^{a} \hat{\rho}_{a}\right)^{n}\right] .
$$

To compute (F.9), we need to evaluate the spectral traces

$$
j_{a_{1}, \cdots, a_{n}} \equiv \operatorname{Tr}\left(\hat{\rho}_{a_{1}} \cdots \hat{\rho}_{a_{n}}\right),
$$

which can be further decomposed as

$$
j_{a_{1}, \cdots, a_{n}}=\sum_{\epsilon_{k}= \pm} \operatorname{Tr}\left(\hat{\rho}_{a_{1} \epsilon_{1}} \cdots \hat{\rho}_{a_{n} \epsilon_{n}}\right)
$$

As mentioned above, one needs to take into account the pinching of the contour to obtain the correct result. One important difference from the analysis in the main text is that, for most of the choices of the signs $\epsilon_{k}$, in (F.11), there are some contours which are not pinched in the limit. When this happens, the contribution coming from such an integral will become subleading. The only ases where all the contours are pinched are the ones with $\epsilon_{k}=+$ for all $k$ or $\epsilon_{k}=-$ for all $k$. Thus, the leading result in the M-theory limit is given by

$$
j_{a_{1}, \cdots, a_{n}} \sim \operatorname{Tr}\left(\rho_{a_{1}+} \cdots \rho_{a_{n}+}\right)+\operatorname{Tr}\left(\rho_{a_{1}-} \cdots \rho_{a_{n}-}\right) .
$$


The contributions from these two terms can be determined using the clustering method explained in the main text and the result reads

$$
\begin{aligned}
j_{a_{1}, \cdots, a_{n}} & \sim \frac{1}{k \pi \sum_{i=1}^{n}\left(a_{i}+1 / 2\right)} \int \frac{d x}{2 \pi} \frac{1}{\left(2 \cosh \frac{x}{2}\right)^{n}} . \\
\ln \Theta & \sim \sum_{n=1}^{\infty} \frac{(-1)^{n-1} c_{n}}{k \pi n} \int \frac{d x}{2 \pi}\left(\frac{z}{2 \cosh \frac{x}{2}}\right)^{n},
\end{aligned}
$$

with the constant $c_{n}$ given by

$$
c_{n}=\sum_{a_{1}=0}^{\infty} \cdots \sum_{a_{n}=0}^{\infty} \frac{(-1)^{\sum_{i=1}^{n} a_{i}}}{\sum_{i=1}^{n}\left(a_{i}+1 / 2\right)} .
$$

To compute this sum, we convert the summand to the following integral

$$
c_{n}=\sum_{a_{1}=0}^{\infty} \cdots \sum_{a_{n}=0}^{\infty}(-1)^{\sum_{i=1}^{n} a_{i}} \int_{0}^{\infty} d p e^{-p \sum_{i=1}^{n}\left(a_{i}+1 / 2\right)} .
$$

Then, exchanging the order of the summations and the integration and performing the sums explicitly, we arrive at

$$
c_{n}=\int_{0}^{\infty} d p\left(\frac{e^{-p / 2}}{1+e^{-p}}\right)^{n}=\int_{-\infty}^{\infty} \frac{d p}{2} \frac{1}{\left(2 \cosh \frac{p}{2}\right)^{n}} .
$$

Now substituting (F.17) into (F.14), we obtain

$$
\begin{aligned}
\ln \Theta & \sim \sum_{n=1}^{\infty} \frac{(-1)^{n-1} z^{n}}{n} \int \frac{d x d p}{2 \pi \hbar}\left(\frac{1}{2 \cosh \frac{x}{2}} \frac{1}{2 \cosh \frac{p}{2}}\right) \\
& =\int \frac{d x d p}{2 \pi \hbar} \ln \left(1+z e^{-H(p, x)}\right)
\end{aligned}
$$

with

$$
\hbar \equiv 2 \pi k, \quad H(p, x) \equiv \ln \left(2 \cosh \frac{p}{2}\right)+\log \left(2 \cosh \frac{x}{2}\right) .
$$

This is precisely the expression derived in [19].

\section{G Separation of variables from clustering}

In this Appendix, we study the integral representation of the tree-level three-point function with one non-BPS operator in the $\mathfrak{s u}(2)$ and the $\mathfrak{s l}(2)$ sectors, and show that they are related to a totally different integral expression, which is based on the separation of variables (SoV) [56, 58]. 


\section{The $\mathfrak{s u}(2)$ sector}

We start from the integral expression ${ }^{22}$,

$$
\mathscr{A}=\sum_{n=0}^{\infty} \frac{\kappa^{n}}{n !} \oint_{\mathcal{C}_{\mathbf{u}}} \prod_{j=1}^{n} \frac{d z_{j}}{2 \pi} f_{\theta}\left(z_{j}\right) \prod_{j<k}^{n} \Delta\left(z_{j}, z_{k}\right)
$$

where, for later convenience, we introduced the twist $\kappa$ and deformed the potential term $f(z)$ as

$$
f_{\theta}(z)=\frac{1}{h(z, \mathbf{u})} \prod_{s=1}^{\ell} \frac{z-\theta_{s}-i / 2}{z-\theta_{s}+i / 2}=\prod_{i=1}^{M} \frac{z-u_{i}+i}{z-u_{i}} \prod_{s=1}^{\ell} \frac{z-\theta_{s}-i / 2}{z-\theta_{s}+i / 2}
$$

As the first step, we rewrite (G.1) using the Cauchy determinant identity as

$$
\mathscr{A}=\sum_{n=0}^{\infty} \frac{\kappa^{n}}{n !} \oint_{\mathcal{C}_{\mathbf{u}}} \prod_{j=1}^{n} \frac{d z_{j}}{2 \pi i} f_{\theta}\left(z_{j}\right) \operatorname{det}\left(\frac{1}{z_{i}-z_{j}-i}\right) .
$$

This expression is similar in form to the Fredholm determinant and therefore the logarithm of $\mathscr{A}$ admits the expansion:

$$
\begin{aligned}
\log \mathscr{A} & =-\sum_{n=1}^{\infty} \frac{(-\kappa)^{n}}{n} W_{n} \\
W_{n} & =\oint_{\mathcal{C}_{\mathbf{u}}} \frac{d^{n} z}{(2 \pi i)^{n}} \rho\left(z_{1}, z_{2}\right) \rho\left(z_{2}, z_{3}\right) \cdots \rho\left(z_{n}, z_{1}\right) .
\end{aligned}
$$

with

$$
\rho(u, v)=\frac{f_{\theta}(u)}{u-v-i}
$$

As (G.4) shows, the integrand of $W_{n}$ contains poles at $z_{j}=\theta_{s}-i / 2$. The basic idea to make connection with the SoV representation is to deform the contours and close them around these poles. Such deformation yields two additional contributions: The one comes from poles in the interaction term $\Delta\left(z_{i}, z_{j}\right)$ and the other comes from poles at infinity.

Let us first examine the contribution from interaction terms. Suppose that we already deformed some of the contours in (G.4) and are in a position to deform yet another contour, say the contour of $z_{k}$. In the process of deformation, the contour of $z_{k}$ first hits the poles at $z_{k}=z_{j}+i n(n \in \mathbb{Z})$, where $z_{j}$ is a variable whose contour is not yet deformed. The poles of this type are precisely the ones considered in section 3.3 and, as discussed there, it

\footnotetext{
${ }^{22}$ In this Appendix, we set $\epsilon=1$.
} 
produces the bound-state contributions. Now, when the contour of $z_{k}$ approahes $\theta_{s}+i / 2$, it hits another set of poles at $z_{k}=z_{m}+i n(n \in \mathbb{Z})$, where this time $z_{m}$ is a variable whose contour is already deformed. Interestingly, in the case of $\mathfrak{s u}(2)$, these two contributions precisely cancel out ${ }^{23}$ with each other. Thus we get

$$
\log \mathscr{A}=\log \tilde{\mathscr{A}}+\log \mathscr{A}_{\infty}
$$

with

$$
\begin{aligned}
\log \tilde{\mathscr{A}} & =-\sum_{n=1}^{\infty} \frac{\kappa^{n}}{n} \tilde{W}_{n} \\
\tilde{W}_{n} & =\oint_{\mathcal{C}_{\theta^{-}}} \frac{d^{n} z}{(2 \pi i)^{n}} \rho\left(z_{1}, z_{2}\right) \rho\left(z_{2}, z_{3}\right) \cdots \rho\left(z_{n}, z_{1}\right) .
\end{aligned}
$$

Here $\mathcal{C}_{\theta^{-}}$is the contour surrounding $\theta_{s}-i / 2$ counterclockwise and log $\mathscr{A}_{\infty}$ denotes the contribution from infinity.

We next study the contribution from infinity. To illustrate the basic mechanism, let us consider $W_{2}$,

$$
W_{2}=\oint_{\mathcal{C}_{\mathbf{u}}} \frac{d z_{1} d z_{2}}{(2 \pi i)^{2}} \frac{f_{\theta}\left(z_{1}\right)}{z_{1}-z_{2}-i} \frac{f_{\theta}\left(z_{2}\right)}{z_{2}-z_{1}-i} .
$$

Since the integrand scales as $1 / z_{1}^{2}$ when $z_{1} \sim \infty$, it may seem that the contribution from infinity is completely absent. However, this is actually not true: If we first take the pole of $1 /\left(z_{1}-z_{2}-i\right)$, we arrive at a single integral whose integrand is given by

$$
\frac{1}{2 i} \oint_{\mathcal{C}_{\mathbf{u}}} \frac{d z_{2}}{2 \pi i} f_{\theta}^{++}\left(z_{2}\right) f_{\theta}\left(z_{2}\right)
$$

Then, if we take the residue of this integrand at infinity, we get the contribution $-(\ell-M)$, where $M$ is the number of magnons. Repeating such analysis, we can prove the contribution from infinity for $W_{n}$ is given by

$$
-(-1)^{n}(\ell-M)
$$

Therefore we have

$$
\log \mathscr{A}_{\infty}=(\ell-M) \sum_{n=1}^{\infty} \frac{\kappa^{n}}{n}=\log \left((1-\kappa)^{-(\ell-M)}\right) .
$$

\footnotetext{
${ }^{23}$ One can show this by the combinatorial argument as in section 3.3. Since the argument is similar, we will not repeat it here.
} 
As (G.11) shows, $\mathscr{A}_{\infty}$ is divergent in the zero twist limit $\kappa \rightarrow 1$. As we see later, this divergence is canceled out by a vanishing factor coming from $\tilde{\mathscr{A}}$.

As the next step, we interpret (G.7) as the Fredholm determinant. As mentioned before, the form of (G.7) is similar to the expansion of the Fredholm determinant $\operatorname{det}(I+\kappa \hat{G})$. However, to make the relation more precise, we need to specify the definition of $\hat{G}$ and the Hilbert space it acts on $^{24}$. As the Hilbert space, we take a space of functions with simple poles at $\theta_{i}^{-}(i=1, \ldots, \ell)$ and infinity, $\mathcal{H}_{\theta}$. Obviously it is $\ell$-dimensional Hilbert space and a general vector belonging to $\mathcal{H}_{\theta}$ van be expressed as

$$
F(x) \equiv \sum_{j} \frac{f_{j}}{x-\theta_{j}^{-}} .
$$

The dual vector space $\mathcal{H}^{*}$ is given by a space of cycles spanned by $t_{j}(j=1, \ldots, \ell)$, where $t_{j}$ is the contour around $\theta_{j}^{-}$. The inner product is then defined by

$$
\langle\tilde{F} \mid F\rangle=\oint_{\tilde{F}} \frac{d x}{2 \pi i} F(x) .
$$

When $F$ and $\tilde{F}$ are given by

$$
F(x)=\sum_{j} \frac{f_{j}}{x-\theta_{j}^{-}}, \quad \tilde{F}=\sum_{k} \tilde{f}_{k} t_{k},
$$

the inner product coincides with the standard inner product between two vectors $\left(f_{1}, \ldots, f_{\ell}\right)^{t}$ and $\left(\tilde{f}_{1}, \ldots, \tilde{f}_{\ell}\right)$. Now, consider the operator $\hat{G}$ acting on this function space as

$$
\hat{G} \cdot F(x)=\oint_{\mathcal{C}_{\theta^{-}}} \frac{d y}{2 \pi i} \frac{f_{\theta}(y)}{x-y} F(y+i) .
$$

Then we can check that $\log \operatorname{det}(I+\kappa \hat{G})=\operatorname{Tr} \log (I+\kappa \hat{G})$ indeed yields the series (G.7). Thus in the end the structure constant can be expressed as

$$
\mathscr{A}=\frac{\operatorname{det}(I+\kappa \hat{G})}{(1-\kappa)^{\ell-M}} .
$$

Now, we relate (G.16) to the SoV integral formula. For this purpose, we first decompose the operator $I+\kappa \hat{G}$ as

$$
I+\kappa \hat{G}=\hat{K} \hat{L}
$$

\footnotetext{
${ }^{24}$ This was already clarified in [9] and what we explain below is the minor modification of their construction.
} 
where $\hat{K}$ and $\hat{L}$ are given by

$$
\begin{aligned}
& \hat{K} \cdot F(x)=\oint_{\mathcal{C}_{\theta^{-}}} \frac{d y}{2 \pi i} \frac{e^{-\phi y}}{x-y} \frac{Q_{\theta}^{-}(y)}{Q_{\mathbf{u}}(y)} F(y) \\
& \hat{L} \cdot F(x)=\oint_{\mathcal{C}_{\theta^{-}}} \frac{d y}{2 \pi i} \frac{e^{\phi y}}{x-y} \frac{Q_{\mathbf{u}}(y)}{Q_{\theta}^{-}(y)} F(y)+\oint_{\mathcal{C}_{\theta^{-}}} \frac{d y}{2 \pi} \frac{e^{\phi(y+i)}}{x-y} \frac{Q_{\mathbf{u}}^{++}(y)}{Q_{\theta}^{+}(y)} F(y+i)
\end{aligned}
$$

Here $Q_{\mathbf{u}}$ and $Q_{\theta}$ are the Baxter polynomials defined by

$$
Q_{\mathbf{u}}(u)=\prod_{i=1}^{M}\left(u-u_{i}\right), \quad Q_{\theta}(u)=\prod_{s=1}^{\ell}\left(u-\theta_{s}\right)
$$

and $\phi$ is related to the twist by $e^{i \phi}=-\kappa$. Since the operator $\hat{K}$ acts diagonally on the basis $\left\{1 /\left(x-\theta_{1}^{-}\right), \ldots, 1 /\left(x-\theta_{\ell}^{-}\right)\right\}$, its determinant can be easily computed as

$$
\operatorname{det} \hat{K}=e^{-\phi \sum_{s} \theta_{s}^{-}} \frac{\prod_{s, t}\left(\theta_{s}-\theta_{t}-i\right)}{\prod_{s, i}\left(\theta_{s}-u_{i}-i / 2\right)}
$$

To compute the determinant of $\hat{L}$, we first express the matrix element of $\hat{L}$ in the basis

$$
\left\{\frac{1}{Q_{\theta}^{+}(x)}, \frac{x}{Q_{\theta}^{+}(x)}, \ldots, \frac{x^{\ell-1}}{Q_{\theta}^{+}(x)}\right\} \text {. }
$$

Then we get

$$
\begin{aligned}
(\hat{L})_{n, m} & =\oint_{\theta_{n}^{-}} \frac{d x}{2 \pi i} \frac{e^{\phi x} Q_{\mathbf{u}}(x) x^{m-1}}{Q_{\theta}^{+}(x) Q_{\theta}^{-}(x)}+\oint_{\theta_{n}^{-}} \frac{d x}{2 \pi i} \frac{e^{\phi(x+i)} Q_{\mathbf{u}}^{++}(x)(x+i)^{m-1}}{Q_{\theta}^{++}(x) Q_{\theta}^{+}(x)} \\
& =\oint_{\theta_{n}^{-} \cup \theta_{n}^{+}} \frac{d x}{2 \pi i} \frac{e^{\phi x} Q_{\mathbf{u}}(x) x^{m-1}}{Q_{\theta}^{+}(x) Q_{\theta}^{-}(x)} .
\end{aligned}
$$

Now using the Vandermonde determinant formula, we can express the determinant of $\hat{L}$ as

$$
\operatorname{det} \hat{L}=J\left(\prod_{n=1}^{\ell} \oint_{\theta_{n}^{-} \cup \theta_{n}^{+}} \frac{d x_{n}}{2 \pi i}\right) \prod_{m=1}^{\ell} \frac{e^{\phi x_{m}} Q_{\mathbf{u}}\left(x_{m}\right)}{Q_{\theta}^{+}\left(x_{m}\right) Q_{\theta}^{-}\left(x_{m}\right)} \prod_{i<j}\left(x_{j}-x_{i}\right) .
$$

where $J$ is the Jacobian for the change of basis, which is given by

$$
J^{-1}=\operatorname{det}\left(\oint_{\theta_{n}^{-}} \frac{d x}{2 \pi i} \frac{x^{m}}{Q_{\theta}^{+}(x)}\right)_{n, m}=\frac{1}{\prod_{i<j}\left(\theta_{j}-\theta_{i}\right)} .
$$

The expression (G.23) is already very similar to the SoV integral formula. However, there are two important differences. First, in the SoV formula derived in [56], the twist $\phi$ is set to zero. Second, the number of integration variables in the SoV formula is $\ell-1$ 
whereas here we have $\ell$. Thus, to obtain the SoV formula, we need to integrate out one of the variables sending $\phi \rightarrow 0$. For this purpose, we first change the integration contour of (G.23) to $\mathcal{C}_{\theta^{ \pm}}$, which surround all the $\theta_{s} \pm i / 2$, at the cost of introducing an extra factor in the integrand ${ }^{25}$ :

$$
\operatorname{det} \hat{L}=\frac{J}{\prod_{i<j}\left(e^{2 \pi \theta_{j}}-e^{2 \pi \theta_{i}}\right)} \oint_{\mathcal{C}_{\theta^{-}}} \prod_{n=1}^{\ell} \frac{d x_{n}}{2 \pi i} \frac{e^{\phi x_{n}} Q_{\mathbf{u}}\left(x_{n}\right)}{Q_{\theta}^{+}\left(x_{n}\right) Q_{\theta}^{-}\left(x_{n}\right)} \prod_{i<j}\left(e^{2 \pi x_{j}}-e^{2 \pi x_{i}}\right)\left(x_{j}-x_{i}\right)
$$

Now using the Vandermonde determinant formula again, we can convert this multiple integral to the following determinant:

$$
\begin{aligned}
\operatorname{det} \hat{L} & =\frac{J}{\prod_{i<j}\left(e^{2 \pi \theta_{j}}-e^{2 \pi \theta_{i}}\right)} \operatorname{det} M_{n, m}, \\
M_{n, m} & =\oint_{\mathcal{C}_{\theta^{-}}} \frac{d x}{2 \pi i} \frac{e^{\phi x} Q_{\mathbf{u}}(x)}{Q_{\theta}^{+}(x) Q_{\theta}^{-}(x)} x^{m-1} e^{2 \pi(n-1) x} .
\end{aligned}
$$

We then perform the integral of $M_{1, m}$ in the regime $\phi \sim 0$ to get

$$
M_{1, m}=(-\phi)^{2 \ell-M-m}+\cdots
$$

Thus the leading contribution comes from $M_{1, \ell}$ and this precisely cancels the divergent factor $\mathscr{A}_{\infty}$ given by (G.11). As a result, the remaining contribution is given by the subdeterminant where the first row and the $\ell$-th column are omitted. Converting this sub-determinant back to the integral, we arrive at the following expression for the structure constant,

$$
\begin{aligned}
& \mathscr{A}=C \oint_{\mathcal{C}_{\theta^{ \pm}}} \prod_{n} \frac{d x_{n}}{2 \pi i} \frac{Q_{\mathbf{u}}\left(x_{n}\right) e^{2 \pi x_{n}}}{Q_{\theta}^{+}\left(x_{n}\right) Q_{\theta}^{-}\left(x_{n}\right)} \prod_{k<l}\left(x_{k}-x_{l}\right)\left(e^{2 \pi x_{k}}-e^{2 \pi x_{l}}\right), \\
& C=e^{-\phi \sum_{s} \theta_{s}^{-}} \frac{\prod_{s, t}\left(\theta_{s}-\theta_{t}-i\right)}{\prod_{s, i}\left(\theta_{s}-u_{i}-i / 2\right)} \prod_{i<j} \frac{\left(\theta_{i}-\theta_{j}\right)}{\left(e^{2 \pi \theta_{i}}-e^{2 \pi \theta_{j}}\right)} .
\end{aligned}
$$

which coincides with the formula in [56].

\section{The $\mathfrak{s l}(2)$ sector}

We next study the $\mathfrak{s l}(2)$ sector,

$$
\mathscr{A}=\sum_{n=0}^{\infty} \frac{(-\kappa)^{n}}{n !} \oint_{\mathcal{C}_{\mathbf{u}}} \prod_{j=1}^{n} \frac{d z_{j}}{2 \pi} f_{\theta}^{\mathfrak{s l}(2)}\left(z_{j}\right) \prod_{j<k}^{n} \Delta\left(z_{j}, z_{k}\right)
$$

\footnotetext{
${ }^{25}$ See [56] for details.
} 
where $f_{\theta}^{\mathfrak{s l}(2)}$ in this case is given by

$$
f_{\theta}^{\mathfrak{s l}(2)}(z)=\frac{1}{h_{\mathfrak{s l}(2)}(z, \mathbf{u})} \prod_{s=1}^{\ell} \frac{z-\theta_{s}-i / 2}{z-\theta_{s}+i / 2}=\prod_{i=1}^{M} \frac{z-u_{i}-i}{z-u_{i}} \prod_{s=1}^{\ell} \frac{z-\theta_{s}-i / 2}{z-\theta_{s}+i / 2} .
$$

The basic strategy is the same as in the $\mathfrak{s u}(2)$ sector. Namely, we write down the expansion for $\log \mathscr{A}$ as in (G.4) and deform the contours. The important difference is that unlike the $\mathfrak{s u}(2)$ sector, the contributions from poles in the interaction term do not cancel out each other but they add up. By the straightforward (but complicated and tedious) computation, we arrive at the following expression:

$$
\mathscr{A}=(1-\kappa)^{\ell+M} \sum_{\vec{n}} \frac{(-\kappa)^{\sum_{a} n_{a}} \tilde{B}[\vec{n}]}{\prod_{a} n_{a} !}
$$

Here the factor $(1-\kappa)^{\ell+M}$ comes from poles at infinity as in the $\mathfrak{s u}(2)$ case and the contribution from each configurartion $\vec{n}$ is given by

$$
\tilde{B}[\vec{n}]=\oint_{\mathcal{C}_{\theta^{-}}} \prod_{a} \prod_{j=1}^{n_{a}} \frac{d z_{j}^{a}}{2 \pi a i} f_{\theta ; a}^{\mathfrak{s l}(2)}\left(z_{j}^{a}\right) \prod_{\substack{a \\ a \leq i<j \leq n_{a}}} \Delta_{a, a}\left(z_{i}^{a}, z_{j}^{a}\right) \prod_{\substack{a<b \\ 1 \leq i \leq n_{a} \\ 1 \leq j \leq n_{b}}} \Delta_{a, b}\left(z_{i}^{a}, z_{j}^{b}\right)
$$

where $\Delta_{a, b}$ are defined by (3.19) and $f_{\theta ; a}$ is given by

$$
f_{\theta ; a}^{\mathfrak{s l}(2)}(z)=\frac{Q_{\mathbf{u}}^{[-2 a]}(z)}{Q_{\mathbf{u}}(z)} \frac{Q_{\theta}^{[1-2 a]}(z)}{Q_{\theta}^{+}(z)}
$$

Now, as in section 6.6, we can convert this to the following generalised Fredholm determinant $^{26}$ :

$$
\mathscr{A}=(1-\kappa)^{\ell+M} \sum_{\vec{n}} \operatorname{det}\left(I+\sum_{a=1}^{\infty}(-\kappa)^{a} \hat{G}_{a}\right)
$$

Here $\hat{G}_{a}$ is the operator acting on the space $\mathcal{H}$ as

$$
\hat{G}_{a} \cdot F(x)=\oint_{\mathcal{C}_{\theta}^{-}} \frac{d y}{2 \pi i} \frac{f_{\theta ; a}^{\mathfrak{s l}(2)}(y)}{x-y} F(y-a i)
$$

As in the $\mathfrak{s u}(2)$ sector, we can decompose the operator $I+\sum_{a} \kappa^{a} \hat{G}_{a}$ as

$$
I+\sum_{a} \kappa^{a} \hat{G}_{a}=\hat{K}_{\mathfrak{s l}(2)} \hat{L}_{\mathfrak{s l}(2)}
$$

\footnotetext{
${ }^{26}$ This can be verified by expanding the generalised Fredholm determinant and compareing with the series (G.31).
} 
where $\hat{K}_{\mathfrak{s l}(2)}$ and $\hat{L}_{\mathfrak{s l}(2)}$ are given by

$$
\begin{aligned}
\hat{K}_{\mathfrak{s l}(2)} \cdot F(x)= & \oint_{\mathcal{C}_{\theta^{-}}} \frac{d y}{2 \pi i} \frac{e^{(\phi-\pi) y}}{x-y} \frac{\cosh _{Q_{\theta}}(y)}{Q_{\mathbf{u}}(y) Q_{\theta}^{+}(y)}, \\
\hat{L}_{\mathfrak{s l}(2)} \cdot F(x)= & \oint_{\mathcal{C}_{\theta^{-}}} \frac{d y}{2 \pi i} \frac{e^{-(\phi-\pi) y}}{x-y} \frac{Q_{\mathbf{u}}(y) Q_{\theta}^{+}(y)}{\cosh _{Q_{\theta}}(y)} F(y) \\
& +\sum_{a=1}^{\infty} \oint_{\mathcal{C}_{\theta^{-}}} \frac{d y}{2 \pi i} \frac{e^{-(\phi-\pi)(y-a i)}}{x-y} \frac{Q_{\mathbf{u}}^{[2 a]}(y) Q_{\theta}^{[1-2 a]}(y)}{\cosh _{Q_{\theta}}(y)} F(y-a i)
\end{aligned}
$$

where $\cosh _{Q_{\theta}}(z)$ denotes

$$
\cosh _{Q_{\theta}}(z)=\prod_{s} \cosh \pi\left(z-\theta_{s}\right)
$$

As in the previous case, the determinant of $\hat{K}_{\mathfrak{s l}(2)}$ can be computed straightforwardly as follows since it acts diagonally on $\mathcal{H}_{\theta}$ :

$$
\operatorname{det} \hat{K}_{\mathfrak{s l}(2)}=e^{(\phi-\pi) \sum_{s} \theta_{s}^{-}} \frac{1}{\prod_{i, s}\left(\theta_{s}^{-}-u_{i}\right)} \prod_{s \neq t} \frac{\cosh \pi\left(\theta_{s}-\theta_{t}\right)}{\left(\theta_{s}-\theta_{t}\right)}
$$

To compute the determinant of $\hat{L}_{\mathfrak{s l}(2)}$, we again use the basis (G.21). In this basis, the matrix element reads

$$
\left(\hat{L}_{\mathfrak{s l}(2)}\right)_{n, m}=\sum_{a=0}^{\infty} \oint_{\theta_{n}^{[-2 a-1]}} \frac{d x}{2 \pi i} \frac{Q_{\mathbf{u}}(x) e^{-(\phi-\pi) x} x^{m-1}}{\cosh _{Q_{\theta}}(x)} .
$$

Converting this to the multiple integral using the Vandermode determinant formula and changing the integration contours by introducing the factor $\sinh \left(\pi\left(x_{i}-x_{j}\right)\right)$, we obtain

$$
\operatorname{det} \hat{L}_{\mathfrak{s l}(2)}=\frac{J}{\prod_{i<j} \sinh \left(\pi\left(\theta_{j}-\theta_{i}\right)\right)} \oint_{\tilde{\mathcal{C}}_{\theta}} \prod_{n=1}^{\ell} \frac{d x_{n}}{2 \pi i} \frac{e^{-(\phi-\pi) x_{n}} Q_{\mathbf{u}}\left(x_{n}\right)}{\cosh _{Q_{\theta}}\left(x_{n}\right)} \prod_{i<j} \sinh \left(\pi\left(x_{i}-x_{j}\right)\right)\left(x_{j}-x_{i}\right),
$$

where $\tilde{C}_{\theta}$ is the contour which encircles all the $\theta_{s}-i(a+1 / 2)$ with $a>0$. Now, assuming that $\phi$ has a small negative imaginary part, we can deform the contour $\tilde{\mathcal{C}}_{\theta}$ to the one along the real axis (see figure G.10). Then we get

$$
\operatorname{det} \hat{L}_{\mathfrak{s}(2)}=\frac{(-1)^{\ell} J}{\prod_{i<j} \sinh \left(\pi\left(\theta_{j}-\theta_{i}\right)\right)} \int_{-\infty}^{\infty} \prod_{n=1}^{\ell} \frac{d x_{n}}{2 \pi i} \frac{e^{-(\phi-\pi) x_{n}} Q_{\mathbf{u}}\left(x_{n}\right)}{\cosh _{Q_{\theta}}\left(x_{n}\right)} \prod_{i<j} \sinh \left(\pi\left(x_{i}-x_{j}\right)\right)\left(x_{j}-x_{i}\right),
$$




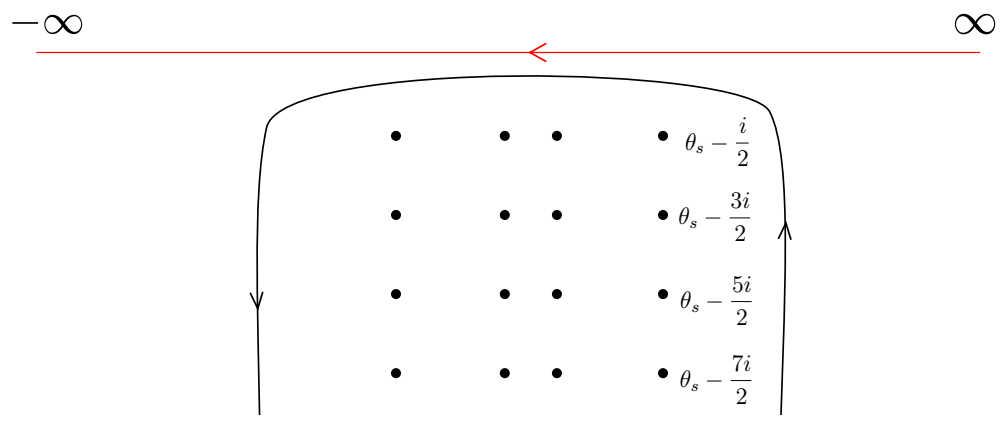

Figure G.10: The deformation of the contour. Initially, the contour encircles counterclockwise $\theta_{s}-i(a+1 / 2)$ with $a \geq 0$. The contour after the deformation is denoted in red and it runs along the real axis from $\infty$ to $-\infty$. $(-1)^{\ell}$ in the formula comes from the change of the direction of the contour.

To take the limit $\phi \rightarrow 0$, we rewrite it as

$$
\begin{aligned}
\operatorname{det} \hat{L}_{\mathfrak{s l}(2)} & =\frac{(-1)^{\ell} J}{\prod_{i<j}\left(e^{2 \pi \theta_{j}}-e^{2 \pi \theta_{i}}\right)} \operatorname{det} M_{n, m}^{\mathfrak{s l}(2)}, \\
M_{n, m}^{\mathfrak{s l}(2)} & =\int_{-\infty}^{\infty} \frac{d x}{2 \pi i} \frac{e^{-\phi x} Q_{\mathbf{u}}(x)}{\cosh _{Q_{\theta}}(x)} \frac{x^{m-1} e^{\pi(2 n-\ell) x}}{2^{\ell-1}} .
\end{aligned}
$$

By studying the asymptotic behavior of the integrand, it is easy to verify that only when $n=\ell$ does $M_{n, m}^{\mathfrak{s l}(2)}$ becomes singular in the limit $\phi \rightarrow 0$. Since the divergence comes from $x \sim \infty$, we can approximate the integrand as

$$
\begin{aligned}
M_{\ell, m}^{\mathfrak{s l}(2)} & \sim \int_{0}^{\infty} \frac{d x}{2 \pi i} \frac{Q_{\mathbf{u}}(x)}{\cosh _{Q_{\theta}}(x)} \frac{x^{m-1} e^{(\pi \ell-\phi) x}}{2^{\ell-1}} \\
& \sim \int_{0}^{\infty} \frac{d x}{4 \pi i} Q_{\mathbf{u}}(x) x^{m-1} e^{-\phi x} \\
& \sim \frac{(M+m-1) !}{4 \pi i \phi^{M+m}}
\end{aligned}
$$

Thus, $M_{\ell, \ell}^{\mathfrak{s I}(2)}$ cancels the prefactor in (G.34), when $\ell \rightarrow 0$. As a result, the remaining contribution is given by the subdeterminant where the $\ell$-th row and the $\ell$-th column are omitted.

Converting it back to the integral, we finally arrive at the following expression,

$$
\mathscr{A} \propto \int_{-\infty}^{\infty} \prod_{n=1}^{\ell-1} \frac{d x_{n}}{2 \pi} \frac{Q_{\mathbf{u}}\left(x_{n}\right)}{\cosh _{Q_{\theta}}\left(x_{n}\right)} \prod_{i<j} \sinh \left(\pi\left(x_{i}-x_{j}\right)\right)\left(x_{j}-x_{i}\right) .
$$

Upon setting $\theta_{s}=0$, this reduces to the SoV-integral expression for the spin $1 / 2 \mathfrak{s l}(2)$ chain obtained in [58]. 


\section{References}

[1] B. Basso, S. Komatsu, and P. Vieira, "Structure Constants and Integrable Bootstrap in Planar N=4 SYM Theory," arXiv:1505.06745 [hep-th].

[2] R. A. Janik and A. Wereszczynski, "Correlation functions of three heavy operators: The AdS contribution," JHEP 1112 (2011) 095, arXiv:1109.6262 [hep-th].

[3] Y. Kazama and S. Komatsu, "On holographic three point functions for GKP strings from integrability," JHEP 01 (2012) 110, arXiv:1110.3949 [hep-th]. [Erratum: JHEP06,150(2012)].

[4] Y. Kazama and S. Komatsu, "Wave functions and correlation functions for GKP strings from integrability," JHEP 09 (2012) 022, arXiv:1205.6060 [hep-th].

[5] Y. Kazama and S. Komatsu, "Three-point functions in the $\mathrm{SU}(2)$ sector at strong coupling," JHEP 1403 (2014) 052, arXiv:1312.3727 [hep-th].

[6] N. Gromov, A. Sever, and P. Vieira, "Tailoring Three-Point Functions and Integrability III. Classical Tunneling," JHEP 07 (2012) 044, arXiv:1111. 2349 [hep-th].

[7] I. Kostov, "Classical Limit of the Three-Point Function of N=4 Supersymmetric Yang-Mills Theory from Integrability," Phys. Rev. Lett. 108 (2012) 261604, arXiv:1203.6180 [hep-th].

[8] I. Kostov, "Three-point function of semiclassical states at weak coupling," J. Phys. A45 (2012) 494018, arXiv:1205.4412 [hep-th].

[9] E. Bettelheim and I. Kostov, "Semi-classical analysis of the inner product of Bethe states," J.Phys. A47 (2014) 245401, arXiv:1403.0358 [hep-th].

[10] B. Basso, A. Sever, and P. Vieira, "Spacetime and Flux Tube S-Matrices at Finite Coupling for N=4 Supersymmetric Yang-Mills Theory," Phys.Rev.Lett. 111 no. 9, (2013) 091602, arXiv:1303.1396 [hep-th].

[11] A. V. Belitsky, "Towards NMHV amplitudes at strong coupling," ArXiv e-prints (Sept., 2015), arXiv:1509.06054 [hep-th].

[12] D. Fioravanti, S. Piscaglia, and M. Rossi, "Asymptotic Bethe Ansatz on the GKP vacuum as a defect spin chain: scattering, particles and minimal area Wilson loops," Nucl. Phys. B898 (2015) 301-400, arXiv:1503.08795 [hep-th]. 
[13] N. A. Nekrasov and S. L. Shatashvili, "Quantization of integrable systems and four dimensional gauge theories," 0908.4052. http://arxiv.org/abs/0908. 4052.

[14] J.-E. Bourgine, "Confinement and Mayer cluster expansions," arXiv:1402.1626 [hep-th].

[15] C. Meneghelli and G. Yang, "Mayer-Cluster Expansion of Instanton Partition Functions and Thermodynamic Bethe Ansatz," JHEP 05 (2014) 112, arXiv:1312.4537 [hep-th].

[16] A. Borodin and I. Corwin, "Macdonald processes," Probab. Theo. Rel. Fields 158 (2014) 225-400, arXiv:1111.4408 [math.PR].

[17] A. M. Povolotsky, "On the integrability of zero-range chipping models with factorized steady states," Journal of Physics A Mathematical General 46 (Nov., 2013) 465205, arXiv:1308.3250 [math-ph].

[18] A. Borodin, I. Corwin, L. Petrov, and T. Sasamoto, "Spectral Theory for Interacting Particle Systems Solvable by Coordinate Bethe Ansatz," Communications in Mathematical Physics 339 (Nov., 2015) 1167-1245, arXiv:1407.8534 [math-ph].

[19] M. Mariño and P. Putrov, "ABJM theory as a Fermi gas," Journal of Statistical Mechanics: Theory and Experiment 3 (Mar., 2012) 1, arXiv:1110.4066 [hep-th].

[20] N. Drukker, M. Marino, and P. Putrov, "From weak to strong coupling in abjm theory," 1007.3837. http://arxiv.org/abs/1007.3837.

[21] Y. Kazama, S. Komatsu, and T. Nishimura, "Classical Integrability for Three-point Functions: Cognate Structure at Weak and Strong Couplings," arXiv:1603.03164 [hep-th].

[22] S. Codesido, A. Grassi, and M. Marino, "Spectral Theory and Mirror Curves of Higher Genus," arXiv:1507.02096 [hep-th].

[23] J. Maldacena, "The Large-N Limit of Superconformal Field Theories and Supergravity," Inter. J. Theor. Phys. 38 (1999) 1113-1133, hep-th/9711200.

[24] J. Escobedo, N. Gromov, A. Sever, and P. Vieira, "Tailoring three-point functions and integrability," JHEP 9 (2011) 28, arXiv:1012.2475.

[25] B. Basso, V. Goncalves, S. Komatsu, and P. Vieira, "Gluing Hexagons at Three Loops," arXiv:1510.01683 [hep-th]. 
[26] N. Beisert, "The Analytic Bethe Ansatz for a Chain with Centrally Extended su(2-2) Symmetry," J. Stat. Mech. 0701 (2007) P01017, arXiv:nlin/0610017 [nlin.SI].

[27] Y. Kazama, S. Komatsu, and T. Nishimura, "Novel construction and the monodromy relation for three-point functions at weak coupling," JHEP 1501 (2015) 095, arXiv:1410.8533 [hep-th].

[28] N. Drukker and J. Plefka, "Superprotected n-point correlation functions of local operators in N=4 super Yang-Mills," JHEP 0904 (2009) 052, arXiv:0901. 3653 [hep-th].

[29] N. Gromov, D. Serban, I. Shenderovich, and D. Volin, "Quantum folded string and integrability: from finite size effects to konishi dimension," 1102.1040. http://arxiv.org/abs/1102.1040.

[30] Y. Jiang and A. Petrovskii, "Diagonal form factors and hexagon form factors," ArXiv e-prints (Nov., 2015), arXiv:1511.06199 [hep-th].

[31] Y. Jiang, "Diagonal Form Factors and Hexagon Form Factors II. Non-BPS Light Operator," ArXiv e-prints (Jan., 2016), arXiv:1601.06926 [hep-th].

[32] N. Beisert, R. Hernandez, and E. Lopez, "A crossing-symmetric phase for AdS(5) x S**5 strings," JHEP 11 (2006) 070, arXiv:hep-th/0609044.

[33] N. Beisert, B. Eden, and M. Staudacher, "Transcendentality and crossing," J. Stat. Mech. 0701 (2007) P021, arXiv:hep-th/0610251.

[34] G. W. Moore, N. Nekrasov, and S. Shatashvili, "D particle bound states and generalized instantons," Commun.Math.Phys. 209 (2000) 77-95, arXiv:hep-th/9803265 [hep-th].

[35] V. Kazakov, I. Kostov, and N. A. Nekrasov, "D-particles, matrix integrals and KP hierarchy," Nucl. Phys. B557 (1999) 413-442, arXiv: hep-th/9810035.

[36] B. Sutherland, "Low-lying eigenstates of the one-dimensional heisenberg ferromagnet for any magnetization and momentum," Phys. Rev. Lett. 74 (Jan, 1995) 816-819. http://link.aps.org/doi/10.1103/PhysRevLett.74.816.

[37] N. Beisert, J. A. Minahan, M. Staudacher, and K. Zarembo, "Stringing spins and spinning strings," JHEP 09 (2003) 010, arXiv:hep-th/0306139. 
[38] N. Dorey, D. M. Hofman, and J. M. Maldacena, "On the Singularities of the Magnon S-matrix," Phys.Rev. D76 (2007) 025011, arXiv:hep-th/0703104 [HEP-TH].

[39] N. Beisert, V. A. Kazakov, K. Sakai, and K. Zarembo, "The Algebraic Curve of Classical Superstrings on $\mathrm{AdS}_{5} \times S^{5}$," Communications in Mathematical Physics 263 (May, 2006) 659-710, hep-th/0502226.

[40] S. Schäfer-Nameki, "Review of AdS/CFT Integrability, Chapter II.4: The Spectral Curve," Letters in Mathematical Physics 99 (Jan., 2012) 169-190, arXiv:1012.3989 [hep-th].

[41] G. Arutyunov and S. Frolov, "The dressing factor and crossing equations," Journal of Physics A Mathematical General 42 (Oct., 2009) 425401, arXiv:0904.4575 [hep-th].

[42] P. Vieira and D. Volin, "Review of ads/cft integrability, chapter iii.3: The dressing factor," 1012.3992. http://arxiv.org/abs/1012.3992.

[43] Z. Tsuboi, "Analytic bethe ansatz and functional relations related to tensor-like representations of type-ii lie superalgebras b $(\mathrm{r}-\mathrm{s})$ and $\mathrm{d}(\mathrm{r}-\mathrm{s})$," Journal of Physics A: Mathematical and General 32 no. 41, (1999) 7175. http://stacks. iop.org/0305-4470/32/i=41/a=311.

[44] V. Kazakov, A. Sorin, and A. Zabrodin, "Supersymmetric Bethe ansatz and Baxter equations from discrete Hirota dynamics," Nucl. Phys. B790 (2008) 345-413, arXiv:hep-th/0703147.

[45] A. Zabrodin, "Backlund transformations for difference Hirota equation and supersymmetric Bethe ansatz," arXiv:0705.4006 [hep-th].

[46] N. Gromov, "Y-system and Quasi-Classical Strings," JHEP 01 (2010) 112, arXiv:0910.3608 [hep-th].

[47] D. Serban, "Integrability and the AdS/CFT correspondence," J.Phys. A44 (2011) 124001, arXiv:1003.4214 [hep-th].

[48] I. Kostov and Y. Matsuo, "Inner products of Bethe states as partial domain wall partition functions," JHEP 10 no. 168, (July, 2012) 1-14, arXiv:1207.2562 [hep-th].

[49] O. Foda and M. Wheeler, "Slavnov determinants, Yang-Mills structure constants, and discrete KP," arXiv:1203.5621 [hep-th]. 
[50] I. Kostov, "Semi-classical scalar products in the generalised SU(2) model," in Lie Theory and Its Applications in Physics: Varna, Bulgaria, June 2013. Springer Japan, Apr., 2014. arXiv:1404.0235 [math-ph].

[51] M. Gaudin, "Une famille à un paramètre d'ensembles unitaires," Nuclear Physics 85 no. 3, (1966) $545-575$. http://www.sciencedirect.com/science/article/pii/0029558266903221.

[52] E. Wigner, "On the Quantum Correction For Thermodynamic Equilibrium," Physical Review 40 no. 5, (June, 1932) 749-759. http://dx.doi.org/10.1103/physrev.40.749.

[53] J. G. Kirkwood, "Quantum statistics of almost classical assemblies," Phys. Rev. 44 (Jul, 1933) 31-37. http://link.aps.org/doi/10.1103/PhysRev.44.31.

[54] A. Dhar, G. Mandal, and S. R. Wadia, "Nonrelativistic fermions, coadjoint orbits of W(infinity) and string field theory at c $=1$," Mod. Phys. Lett. A7 (1992) 3129-3146, arXiv:hep-th/9207011 [hep-th].

[55] I. Kostov, "Conformal field theory techniques in random matrix models," arXiv:hep-th/9907060.

[56] Y. Kazama, S. Komatsu, and T. Nishimura, "A new integral representation for the scalar products of Bethe states for the XXX spin chain," JHEP 09 no. 013, (2013) 1-23, arXiv:1304.5011 [hep-th].

[57] A. Kapustin, B. Willett, and I. Yaakov, "Exact Results for Wilson Loops in Superconformal Chern-Simons Theories with Matter," JHEP 03 (2010) 089, arXiv:0909.4559 [hep-th].

[58] S. Derkachov, G. Korchemsky, and A. Manashov, "Separation of variables for the quantum $S L(2, \mathbb{R})$ spin chain," JHEP 0307 (2003) 047, arXiv: hep-th/0210216v2. 